\title{
Dynamic Game and Coordination Strategy of Multichannel Supply Chain Based on Brand Competition
}

\author{
Junhai Ma $\mathbb{D},{ }^{1}$ Fang Zhang $\left(\mathbb{D},{ }^{1,2}\right.$ and Binshuo Bao $\mathbb{D}^{1}$ \\ ${ }^{1}$ College of Management and Economics, Tianjin University, Tianjin 300072, China \\ ${ }^{2}$ Department of Mathematics, Tianjin Polytechnic University, Tianjin 300387, China \\ Correspondence should be addressed to Fang Zhang; zhanfangxs@163.com and Binshuo Bao; baobinshu01024@163.com
}

Received 10 May 2019; Revised 14 August 2019; Accepted 20 September 2019; Published 5 December 2019

Guest Editor: Raúl Baños

Copyright (c) 2019 Junhai Ma et al. This is an open access article distributed under the Creative Commons Attribution License, which permits unrestricted use, distribution, and reproduction in any medium, provided the original work is properly cited.

In this paper, two noncooperative dynamic pricing strategies are used in a supply chain. Two dynamic Stackelberg game models have been built involving both a manufacturer and a retailer assumed to be the leader in order. In the two models, the manufacturer sells national-brand (NB) product to an independent retailer or directly to consumers through a direct channel. The retailers sell a store-brand (SB) product when they sell the NB product coming from the manufacturer. Thus, there is competition both in different channels and in products with different brands. To analyze the complexity of the model, parameter bifurcation diagrams and strange attractor diagrams have been therefore plotted. The results show that the game leader has advantages when the market is stable, but it turns disadvantageous if the state falls into unstable as the game follower can quickly adjust the strategy to seize the market. The wholesale price and the direct selling price are high that they incur larger profits if the manufacturer is dominant, but it gets worse when the adjustment speed increases. While in the model where the retailer plays a dominant role, the increase in the adjustment speed is unfavorable to retailer. By controlling the total cost of the direct channel and increasing channel competition strength and brand competition strength, the manufacturers can increase their profits in the game dominated by the retailer. In addition, the stable region within the system will be narrow since the market is sensitive to the channel competition, brand competition, and advertising indifference.

\section{Introduction}

The e-commerce has already been rapidly developed and widely applied with the spread of the Internet around the world. More and more manufacturing enterprises set up their own network of direct sales channels; thus, their hybrid channels include both traditional distribution channels and e-channels. These network platforms can help the manufacturers to meet the demands of consumers without the hands of retailers.

A considerable amount of study has been done on a dualchannel supply chain. For instance, with the consideration of shortage caused by random yields, Xiao and Shi [1] establish a dual-channel supply chain framework and study the pricing and channel priority strategy. They find it is the decentralization that really counts in determining the channel priority strategy. Kurata et al. [2] analyze the competition in multiple distribution channels. They explore cross-brand and cross-channel pricing policies and find that brand loyalty building is profitable for both a national-brand and a store-brand. Cai et al. [3] study a dual-channel supply chain game with a price discount. They show that a consistent pricing scheme can reduce the channel conflict by inducing more profit to the retailer. To investigate the optimal decisions for a dual-channel, Soleimani et al. [4] discuss the pricing strategies both in centralized and decentralized scenarios considering production cost disruptions. They figure out the optimal decisions and reveal that if the demand disruption (cost disruption) is enhanced, the optimal prices will also be increased for a given cost disruption (demand disruption). Also by researching a dualchannel model, Batarfi et al. [5] point out dual-channel strategy is better off than the single-channel strategy. Liu and Xiao [6] build a dyadic closed-loop supply chain based on green consumer and corporate social responsibility in which consumers and firms show environmental responsibility 
behaviors. The price and collection rate decision and reverse channel strategy are analyzed. Zheng et al. [7] build a dualchannel closed loop supply chain that consists of a manufacturer, a retailer, and a collector. The effect of forward channel competition and power structure is investigated, and a centralized and three decentralized models are analyzed. He et al. [8] construct a dual-channel closed loop supply chain in which manufacturers can distribute new products by independent retailers and sell remanufactured products by a third part enterprise. The channel structure and pricing decisions for the manufacturer and government's subsidy with competing remanufactured products are discussed. Giri and Dey [9] extend a closed-loop supply chain which included one collector, one recycler, and one manufacturer with a backup supplier considering uncertainty of collection of used products. And the performance of the model increases compared to that of the conditional closed loop supply chain.

Many research studies show that if the manufacturers set up a direct channel to sell products, they can improve their profits largely, but this behavior can reduce the profit of the retailer. Hence, retailer will in turn take some measures to reduce the loss. The retailers often sell a store-brand (SB) product while they sell the national-brand (NB) product coming from the manufacturer. SB products and NB products compete in the market. Existing literatures [10-13] show that when retailers provide the SB products, they can not only reduce the dependence to the $\mathrm{NB}$ products but also enhance the market competitiveness. A previous research [10] studies the competition between many NB products and a single SB product. Narasimhan and Wilcox [11] calculate the pricing decision and the corresponding changes of the profit of the manufacturers and retailers when they provide the SB product in the market. The article points out that when retailers do not sell their SB products, leading manufacturers can make the retailers' profit down to zero through setting a wholesale price, so the retailer has to minimize losses by selling SB products. The literature [12] points out that selling the SB products can weaken the "double marginal effect." Tyagi [13] indicates that introducing the SB products is good for retailers but is unfavorable for manufacturers.

The aforementioned studies make some research on introducing the SB products but has seldom discussed the two problems in one thesis: multichannel competition and the NB product and the SB product competition. In addition, there has been no literature using dynamic game theory to discuss the aforementioned competition in the supply chain. The multistage dynamic repeated game model combined with system dynamics theory has been studied in some literature recently. Zhang and Ma [14] considered two different pricing policies in a dual-channel supply chain with a fair caring retailer. Many dynamic behaviors, such as the bifurcation, periodic cycles, and strange attractors of the systems, are shown in their papers. In this paper, we consider a dynamic pricing model in which the manufacturer has the option of exploiting the direct channel to compete with the retail channel, whereas the retailer has the option of introducing a SB product to compete with the NB product. In order to improve the competitiveness, the retailer will advertise for the SB product. Two noncooperative Stackelberg game models have been established: in one the manufacturer acts as the leader and in the other the retailer acts as the leader. We carried out numerical simulations on two models with different conditions and analyzed the complex dynamic behaviors, such as bifurcation and chaos [15-17].

The structure of this paper is arranged in the following order. In Section 2, we introduce the assumptions and notations. Section 3 investigates a Stackelberg model which is led by the manufacturer and reveals numerical simulations for this scenario. In Section 4, we discuss a retailer leading game framework. In Section 5, a comparison of the prices and profits between the two models are exhibited. Finally, some conclusions have been shown.

\section{Model Notations and Assumptions}

In this paper, we study a multichannel supply chain which includes a manufacturer and a retailer. The manufacturers produces a national-brand (NB) product at a marginal $\operatorname{cost} c_{1}$ and distributes it through their wholly owned direct channel at a price $p_{0}$ as well as an independent retailer channel at a wholesale price $w$. The retailers will resell the product through their own channel at price $p_{1}$. At the same time, the retailers sell the SB product to the customers at a price $p_{2}$. Different products and different channels compete in the supply chain. We develop two noncooperative Stackelberg game models. The supply chain is shown in Figure 1.

We consider the demand for products in the market as a linear function of the product price because linear demand functions are tractable and widely used in supply chain analysis (Ma and Xie [18]; Dai et al. [19]; and Yao et al. [20]). Assume that consumers are divided into two categories: manufacturer loyalty consumers and retailer loyalty consumers. Consumers loyal to the manufacturer buy products only from the manufacturer. Consumers loyal to the retailer choose products from the retailer. Two types of consumers are both sensitive to price. The demands in our models are as follows:

$$
\begin{aligned}
& D_{0}=a_{0}-b_{0} p_{0}+\eta p_{1}, \\
& D_{1}=a_{1}-b_{1} p_{1}+\eta p_{0}+\eta p_{2}-\theta \sqrt{A}, \\
& D_{2}=a_{2}-b_{2} p_{2}+\eta p_{1}+\theta \sqrt{A} .
\end{aligned}
$$

For the convenience, we assume $\sqrt{A}=B$, and then the demand functions are

$$
\begin{aligned}
& D_{0}=a_{0}-b_{0} p_{0}+\eta p_{1} \\
& D_{1}=a_{1}-b_{1} p_{1}+\eta p_{0}+\eta p_{2}-\theta B, \\
& D_{2}=a_{2}-b_{2} p_{2}+\eta p_{1}+\theta B .
\end{aligned}
$$

Here, the parameter $\alpha_{i}(>0)(i=0,1,2)$ represents the potential market size. $b_{i}(>0)(i=0,1,2)$ is the price elasticity coefficient. $\gamma(>0)$ is the channel competition coefficient. $\eta(>0)$ is the brand competition coefficient $b_{i}>\gamma, \eta(\mathrm{i}=0,1,2)$, i.e., the ownership-price effect is greater than the cross-price effect. In addition, $\theta$ is the demand sensitivity to the advertising level of the retailer. The price and demand functions should be satisfied: (i) $c_{1}<w<p_{1}$, (ii) $c_{1}<c_{0}<p_{0}$, (iii) $c_{2}<p_{2}$, and (iv) $D_{i}>0(i=0,1,2)$. 


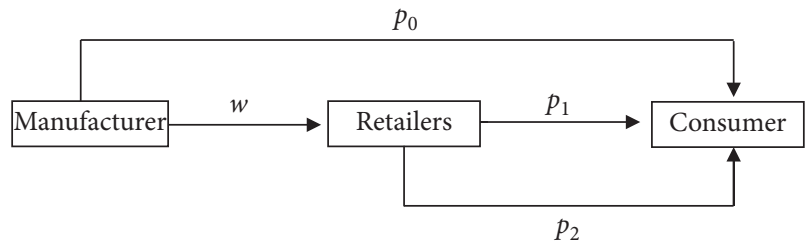

Figure 1

\section{Manufacturer Stackelberg (MS)}

3.1. Model Construction. In this model, the manufacturer is the leader and the retailer is the follower, and they form a Stackelberg game. The manufacturer decides the wholesale price $w$ and the direct selling price $p_{0}$ of the NB product. According to the decision-making of the manufacturer, the retailer makes decision of the retail price $p_{1}$ of the $\mathrm{NB}$ product, the price $p_{2}$ of the SB product, and the advertising investment $\mathrm{A}$.

The profit function of the manufacturer is as follows:

$$
\pi_{R}^{M}=D_{0}\left(p_{0}-c_{0}\right)+D_{1}\left(w-c_{1}\right) .
$$

The profit function of the retailer is as follows:

$$
\pi_{r}^{M}=D_{1}\left(p_{1}-w\right)+D_{2}\left(p_{2}-c_{2}\right)-B^{2} .
$$

Taking the first-order partial derivatives of $\pi_{r}^{M}$ with respect to $p_{1}, p_{2}$, and $B$, respectively, and taking the secondorder derivatives further, we can get the Hessian matrix:

$$
\left(\begin{array}{ccc}
-2 b_{1} & 2 \eta & -\theta \\
2 \eta & -2 b_{2} & \theta \\
-\theta & \theta & -2
\end{array}\right) .
$$

Note that $b_{1} b_{2}>\eta^{2}$ when

$$
-4 b_{1} b_{2}+4 \eta^{2}+b_{1} \theta^{2}+b_{2} \theta^{2}-2 \eta \theta^{2}<0 .
$$

The retailer's profit function is concave and has a unique maximum solution. The retailer's best response to the wholesale price $w$ and the direct sale price $p_{0}$ setting by the manufacturer is given in the following:

$$
\begin{aligned}
& p_{1}=\frac{\left(\left(-4 \eta+\theta^{2}\right)\left(-2 a_{2}-2 b_{2} c_{2}+2 w \eta+c_{2} \theta^{2}-w \theta^{2}\right)-\left(4 b_{2}-\theta^{2}\right)\left(-2\left(a_{1}+b_{1} w+p_{0} \gamma-c_{2} \eta\right)+\left(-c_{2}+w\right) \theta^{2}\right)\right)}{\left(4\left(4 b_{1} b_{2}-4 \eta^{2}-b_{1} \theta^{2}-b_{2} \theta^{2}+2 \eta \theta^{2}\right)\right)}, \\
& p_{2}=\frac{\left(-4 a_{1} \eta-4 p_{0} \gamma \eta+4 c_{2} \eta^{2}+a_{1} \theta^{2}+b_{2} c_{2} \theta^{2}+p_{0} \gamma \theta^{2}-3 c_{2} \eta \theta^{2}+w \eta \theta^{2}+a_{2}\left(-4 b_{1}+\theta^{2}\right)-b_{1}\left(4 b_{2} c_{2}-2 c_{2} \theta^{2}+w \theta^{2}\right)\right)}{\left(2\left(-4 b_{1} b_{2}+4 \eta^{2}+b_{1} \theta^{2}+b_{2} \theta^{2}-2 \eta \theta^{2}\right)\right)}, \\
& B=\frac{\left(-b_{1} b_{2} c_{2}+b_{1} b_{2} w-b_{2} p_{0} \gamma+a_{2}\left(b_{1}-\eta\right)+p_{0} \gamma \eta+c_{2} \eta^{2}-w \eta^{2}+a_{1}\left(-b_{2}+\eta\right)\right) \theta}{\left(4 b_{1} b_{2}-4 \eta^{2}-b_{1} \theta^{2}-b_{2} \theta^{2}+2 \eta \theta^{2}\right)} .
\end{aligned}
$$

Substituting (7)-(9) into (3) and then taking the firstorder partial derivatives of $\pi_{m}$ with respect to $w$ and $p_{0}$, respectively, we can obtain the following

$$
\begin{aligned}
\frac{\partial \pi_{m}^{M}}{\partial w}= & \left(4 c_{0} \gamma \eta^{2}-8 p_{0} \gamma \eta^{2}-4 c_{2} \eta^{3}+2 b_{2} c_{0} \gamma \theta^{2}-2 b_{2} p_{0} \gamma \theta^{2}+a_{2} \eta \theta^{2}-b_{2} c_{2} \eta \theta^{2}-3 c_{0} \gamma \eta \theta^{2}+4 p_{0} \gamma \eta \theta^{2}-c_{1} \eta^{2} \theta^{2}\right. \\
& +c_{2} \eta^{2} \theta^{2}+2 w \eta^{2} \theta^{2}+b_{1}^{2}\left(c_{1}-2 w\right)\left(4 b_{2}-\theta^{2}\right)+a_{1}\left(b_{1}\left(4 b_{2}-\theta^{2}\right)+\eta\left(-4 \eta+\theta^{2}\right)\right) \\
& \left.+b_{1}\left(8 w \eta^{2}-a_{2} \theta^{2}+c_{0} \gamma \theta^{2}-2 p_{0} \gamma \theta^{2}-c_{2} \eta \theta^{2}-4 w \eta \theta^{2}+2 c_{1} \eta\left(-2 \eta+\theta^{2}\right)+b_{2}\left(-4 c_{0} \gamma+8 p_{0} \gamma+4 c_{2} \eta+c_{2} \theta^{2}\right)\right)\right) \\
& \cdot\left(8 b_{1} b_{2}-8 \eta^{2}-2 b_{1} \theta^{2}-2 b_{2} \theta^{2}+4 \eta \theta^{2}\right)^{-1}, \\
\frac{\partial \pi_{m}^{M}}{\partial p_{0}}= & \left(2 b_{0}\left(c_{0}-2 p_{0}\right)\left(4 b_{1} b_{2}-4 \eta^{2}-b_{1} \theta^{2}-b_{2} \theta^{2}+2 \eta \theta^{2}\right)+a_{0}\left(8 b_{1} b_{2}-8 \eta^{2}-2 b_{1} \theta^{2}-2 b_{2} \theta^{2}+4 \eta \theta^{2}\right)\right. \\
& -\gamma\left(4 b_{2} c_{0} \gamma-8 b_{2} p_{0} \gamma-4 a_{2} \eta-4 c_{1} \eta^{2}+8 w \eta^{2}+a_{2} \theta^{2}-b_{2} c_{2} \theta^{2}+2 b_{2} w \theta^{2}-c_{0} \gamma \theta^{2}+2 p_{0} \gamma \theta^{2}+c_{1} \eta \theta^{2}+c_{2} \eta \theta^{2}-4 w \eta \theta^{2}\right. \\
& \left.\left.+b_{1}\left(c_{1}-2 w\right)\left(4 b_{2}-\theta^{2}\right)+a_{1}\left(-4 b_{2}+\theta^{2}\right)\right)\right) \cdot\left(8 b_{1} b_{2}-8 \eta^{2}-2 b_{1} \theta^{2}-2 b_{2} \theta^{2}+4 \eta \theta^{2}\right)^{-1} .
\end{aligned}
$$


Taking the second-order derivatives further, we can get the Hessian matrix:

$$
\left(\begin{array}{cc}
H_{1} & \gamma \\
\gamma & H_{2}
\end{array}\right) .
$$

See note 4 From (6), we have $\theta^{2}\left(b_{1}-\eta\right)^{2}-4 b_{1}\left(b_{1} b_{2}-\right.$ $\left.\eta^{2}\right)<\theta^{2}\left(b_{1}-\eta\right)^{2}-\theta^{2} b_{1}\left(b_{1}+b_{2}-2 \eta\right)<\theta^{2}\left(\eta^{2}-b_{1} b_{2}\right)<0$.

So, while satisfying

$$
\begin{aligned}
& \frac{\theta^{2}\left(b_{1}-\eta\right)^{2}-4 b_{1}\left(b_{1} b_{2}-\eta^{2}\right)}{4 b_{1} b_{2}-4 \eta^{2}-b_{1} \theta^{2}-b_{2} \theta^{2}+2 \eta \theta^{2}}-\gamma \gamma 2 b_{0} \\
& +\frac{\gamma^{2}\left(-4 b_{2}+\theta^{2}\right)}{4 \eta^{2}-4 b_{1} b_{2}+b_{1} \theta^{2}+b_{2} \theta^{2}-2 \eta \theta^{2}}-\gamma \gamma^{2}>0,
\end{aligned}
$$

the manufacturer's profit function is concave and has a unique maximum solution because the Hessian matrix is negative definite. So, we get the optimal wholesale price $w^{M}$ and the optimal direct sale price $p_{0}^{M}$ by letting (10) and (11) be equal to zero. Furthermore, substituting $w^{M}$ and $p_{0}^{M}$ into (7)-(9), we have the optimal retail price $p_{1}^{M}$ and $p_{2}^{M}$ and the optimal advertising investment $A^{M}$. See the appendix for a list of functions $w^{M}, p_{0}^{M}, p_{1}^{M}, p_{2}^{M}$, and $B^{M}$.

Now we develop a dynamic Stackelberg game model. In fact, while making price decision, restricted by the objective conditions such as the decision-making ability, the decision makers cannot get the whole market information and their price decision is also not completely rational. Suppose that the decision in next period can be adjusted with bounded rationality (Ma et al. $[17,21])$ and is based on partial estimation of the marginal profits of the current period; that is, if the marginal profits in period $t$ is positive, the manufacturer will raise the price in period $t+1$. Otherwise, the manufacturer will reduce it. The model can be constructed as follows:

$$
\left\{\begin{array}{l}
\omega(t+1)=\omega(t)+\alpha_{1} \omega(t) \frac{\partial \pi_{m}^{M}\left(\omega, p_{0}\right)}{\partial \omega(t)}, \\
p_{0}(t+1)=p_{0}(t)+\alpha_{2} p_{0}(t) \frac{\partial \pi_{m}^{M}\left(\omega, p_{0}\right)}{\partial p_{0}(t)} .
\end{array}\right.
$$

System (14) gives the manufacturer's dynamic price decision and the decision variables directly relate to the positive parameter $\alpha_{i}(i=1,2)$, which represents the price adjustment speed. $\left(\partial \pi_{m}^{M}\left(w, p_{0}\right)\right) /(\partial w(t))$ and $\left(\partial \pi_{m}^{M}(w\right.$, $\left.\left.p_{0}\right)\right) /\left(\partial p_{0}(t)\right)$ are the marginal profits, and they can be obtained by equations (10) and (11). Furthermore, the retailer's price decision can be obtained by $w(t)$ and $p_{0}(t)$ :

$$
\begin{aligned}
& p_{1}(t)=\frac{\left(\left(-4 \eta+\theta^{2}\right)\left(-2 a_{2}-2 b_{2} c_{2}+2 w(t) \eta+c_{2} \theta^{2}-w(t) \theta^{2}\right)-\left(4 b_{2}-\theta^{2}\right)\left(-2\left(a_{1}+b_{1} w(t)+p_{0}(t) \gamma-c_{2} \eta\right)+\left(-c_{2}+w(t)\right) \theta^{2}\right)\right)}{\left(4\left(4 b_{1} b_{2}-4 \eta^{2}-b_{1} \theta^{2}-b_{2} \theta^{2}+2 \eta \theta^{2}\right)\right)}, \\
& p_{2}(t)=\frac{\left(-4 a_{1} \eta-4 p_{0}(t) \gamma \eta+4 c_{2} \eta^{2}+a_{1} \theta^{2}+b_{2} c_{2} \theta^{2}+p_{0}(t) \gamma \theta^{2}-3 c_{2} \eta \theta^{2}+w(t) \eta \theta^{2}+a_{2}\left(-4 b_{1}+\theta^{2}\right)-b_{1}\left(4 b_{2} c_{2}-2 c_{2} \theta^{2}+w(t) \theta^{2}\right)\right)}{\left(2\left(-4 b_{1} b_{2}+4 \eta^{2}+b_{1} \theta^{2}+b_{2} \theta^{2}-2 \eta \theta^{2}\right)\right)}, \\
& B(t)=\frac{\left(\left(-b_{1} b_{2} c_{2}+b_{1} b_{2} w(t)-b_{2} p_{0}(t) \gamma+a_{2}\left(b_{1}-\eta\right)+p_{0}(t) \gamma \eta+c_{2} \eta^{2}-w(t) \eta^{2}+a_{1}\left(-b_{2}+\eta\right)\right) \theta\right)}{\left(4 b_{1} b_{2}-4 \eta^{2}-b_{1} \theta^{2}-b_{2} \theta^{2}+2 \eta \theta^{2}\right)} .
\end{aligned}
$$

3.2. Model Analysis. We have four equilibrium solutions of system (14), described by

$$
\begin{aligned}
E_{1}= & (0,0) \\
E_{2}= & \left(\left(4 c_{0} \gamma \eta^{2}-4 c_{2} \eta^{3}+2 b_{2} c_{0} \gamma \theta^{2}+a_{2} \eta \theta^{2}-b_{2} c_{2} \eta \theta^{2}-3 c_{0} \gamma \eta \theta^{2}-c_{1} \eta^{2} \theta^{2}+c_{2} \eta^{2} \theta^{2}+b_{1}^{2} c_{1}\left(4 b_{2}-\theta^{2}\right)\right.\right. \\
& \left.+a_{1}\left(b_{1}\left(4 b_{2}-\theta^{2}\right)+\eta\left(-4 \eta+\theta^{2}\right)\right)+b_{1}\left(-\left(a_{2}-c_{0} \gamma+c_{2} \eta\right) \theta^{2}+2 c_{1} \eta\left(-2 \eta+\theta^{2}\right)+b_{2}\left(-4 c_{0} \gamma+c_{2}\left(4 \eta+\theta^{2}\right)\right)\right)\right) \\
& \left.\cdot\left(-2 \eta^{2} \theta^{2}+b_{1}^{2}\left(8 b_{2}-2 \theta^{2}\right)+4 b_{1} \eta\left(-2 \eta+\theta^{2}\right)\right)^{-1}, 0\right) \\
E_{3}= & \left(0,\left(2 b_{0} c_{0}\left(4 b_{1} b_{2}-4 \eta^{2}-b_{1} \theta^{2}-b_{2} \theta^{2}+2 \eta \theta^{2}\right)+a_{0}\left(8 b_{1} b_{2}-8 \eta^{2}-2 b_{1} \theta^{2}-2 b_{2} \theta^{2}+4 \eta \theta^{2}\right)\right.\right. \\
& \left.+\gamma\left(4 a_{1} b_{2}-4 b_{1} b_{2} c_{1}-4 b_{2} c_{0} \gamma+4 a_{2} \eta+4 c_{1} \eta^{2}-a_{1} \theta^{2}-a_{2} \theta^{2}+b_{1} c_{1} \theta^{2}+b_{2} c_{2} \theta^{2}+c_{0} \gamma \theta^{2}-c_{1} \eta \theta^{2}-c_{2} \eta \theta^{2}\right)\right) \\
& \left.\cdot\left(2\left(\gamma^{2}\left(-4 b_{2}+\theta^{2}\right)+b_{0}\left(8 b_{1} b_{2}-8 \eta^{2}-2 b_{1} \theta^{2}-2 b_{2} \theta^{2}+4 \eta \theta^{2}\right)\right)\right)^{-1}\right) \\
E^{M}= & \left(\omega^{M}, p_{0}^{M}\right) .
\end{aligned}
$$


$E_{i}(i=1,2,3)$ are boundary equilibrium solutions, while $E^{M}$ is the only Stackelberg equilibrium solution. Its expression is shown as (A.1) and (A.2) in Appendix. In order to guarantee the practical significance, we assume that all equilibriums are positive.

The Jacobian matrix of system (14) is given by

$$
\begin{aligned}
J & =\left(\begin{array}{cc}
1+\alpha_{1} h_{1} & \alpha_{2} p_{0} \gamma \\
\alpha_{1} w \gamma & 1+\alpha_{2} h_{2}
\end{array}\right), \\
h_{2} & =2 H_{1} \omega+\gamma p_{0}+\frac{1}{2}\left(a_{1}+b_{1} c_{1}+\eta c_{2}-\gamma c_{0}\right)+\frac{\theta^{2}\left(\left(b_{1} b_{2}-\eta^{2}\right)\left(c_{1}+c_{2}\right)+a_{2}\left(\eta-b_{1}\right)+\left(a_{1}+\gamma c_{0}\right)\left(b_{2}-\eta\right)\right)}{2\left(4 b_{1} b_{2}-4 \eta^{2}-b_{1} \theta^{2}-b_{2} \theta^{2}+2 \eta \theta^{2}\right)}, \\
h_{2} & =\frac{2 H_{2} p_{0}+\gamma \omega+a_{0}+b_{0} c_{0}-\gamma\left(4 c_{1}\left(b_{1} b_{2}-\eta^{2}\right)+c_{1} \theta^{2}\left(\eta-b_{1}\right)+c_{2} \theta^{2}\left(\eta-b_{2}\right)+a_{2}\left(\theta^{2}-4 \eta^{2}\right)+\left(4 b_{2}-\theta^{2}\right)\left(\gamma c_{0}-a_{1}\right)\right)}{2\left(4 b_{1} b_{2}-4 \eta^{2}-b_{1} \theta^{2}-b_{2} \theta^{2}+2 \eta \theta^{2}\right)} .
\end{aligned}
$$

The stability of equilibrium points will be determined by the nature of the eigenvalues of the Jacobian matrix evaluated at the corresponding equilibrium points. Then, we substitute the value of $E_{i}(i=1,2,3)$ into (19), and we have the following proposition.

Proposition 1. All the boundary equilibrium points $E_{i}(i=$ $1,2,3)$ are unstable under our assumptions and conditions (6) and (13).

The significance of boundary equilibrium is that all players can predict the emergence of this equilibrium, and it is unprofitable for anyone to deviate from the equilibrium. For enterprises, unstable boundary equilibrium is not conducive to the stability of the overall system. We investigate the stability of $E^{R}$ by utilizing Jury's stability condition [22]:

$$
\begin{aligned}
& \text { (i) } 1+\operatorname{Tr}(J)+\operatorname{Det}(J)>0, \\
& \text { (ii) } 1-\operatorname{Tr}(J)+\operatorname{Det}(J)>0, \\
& \text { (iii) } 1-\operatorname{Det}(J)>0,
\end{aligned}
$$

where $\operatorname{Tr}(J)$ and $\operatorname{Det}(J)$ are the trace and determinant of $J$, respectively. The condition (20) gives a stable region in the place of the adjustment parameters $\alpha_{1}$ and $\alpha_{2}$.

\subsection{The Experimental Calculation and Numerical} Simulation. In this section, numerical simulations are carried to show the influence of parameters. We will research how the adjust parameters, channel competition coefficient, brand competition coefficient, and advertising coefficient affect the system stability domain, the product prices, and the long-term average profit by Matlab simulation software. Based on the model assumptions and conditions (6) and (13), we choose some parameters as follows: $a_{0}=30 ; a_{1}=$ $55 ; a_{2}=35 ; b_{0}=2.2 ; b_{1}=4.05 ; b_{2}=2.35 ; \gamma=0.7 ; \eta=0.6$; $\theta=0.5 ; c_{0}=7 ; c_{1}=5 ;$ and $c_{2}=7$. We have the equilibrium prices of the system: $\omega^{M}=11.406 ; \quad p_{0}^{M}=13.3228$; $p_{1}^{M}=15.0705 ; \quad p_{2}^{M}=13.4116 ; \quad$ and $B^{M}=0.6868$. The manufacturer and retailer's optimal profit of stable domain are $\pi_{m}^{M}=141.49$ and $\pi_{r}^{M}=122.32$.

3.3.1. The Stability Region Diagrams of the System. The stability of the equilibrium $E^{M}$ and the two-dimensional bifurcation diagrams of the system are presented in Figure 2. We assign different colors to different areas, namely, stable steady area (blue), stable cycles of periods 2 (green), 3 (pink), 4(red), 5 (dark blue), 6 (brown), 7 (yellow-green), 8 (red pink), chaos (yellow), and divergence (gray). We can see from the figure that when $0<\alpha_{1}<0.042,0<\alpha_{2}<0.034$ (blue area), the system is stable. The two-dimensional bifurcation diagrams of the system show that they have two paths of the system entering into the chaos. One is that when the parameter $(\alpha 1, \alpha 2)$ located in the region of blue, green, red, and red pink moves to yellow step by step, the system enters into chaos through flip bifurcation. Another case is that the system from 2 cycles enters into chaos through NeimarkSacker bifurcation if the parameters from the green area directly enter into the yellow area.

3.3.2. The Influence of Parameters $\gamma, \eta$, and $\theta$ on the Stable Regions. Figure 3 shows the stable regions of the Stackelberg equilibrium point with different channel competition coefficients $(\gamma)$, different brand competition coefficients $(\eta)$, and different advertising coefficients $(\theta)$. In Figure 3(a), when $\gamma=0.7, \gamma=1.1$, and $\gamma=1.5$, we assign blue, yellow, and green to the stable region, respectively. It shows that the stable region of the system decreases with the increase of $\gamma$. That is to say, the fiercer the channel competition, the more unstable the system. In Figure 3(b), when $\eta=0.6, \eta=$ 1 , and $\eta=1.4$, the stable region is shown by blue, pink, and gray, respectively. We can see from the figure that the stable region of the system decreases with the increase of the brand competition coefficient $\eta$. In Figure 3(c), when $\theta=0.5, \theta=0.9$, and $\theta=1.3$, we assign blue, red, and brown to the stable region, respectively. The figure shows that the more sensitive the market is, the less the stability of the system is. 


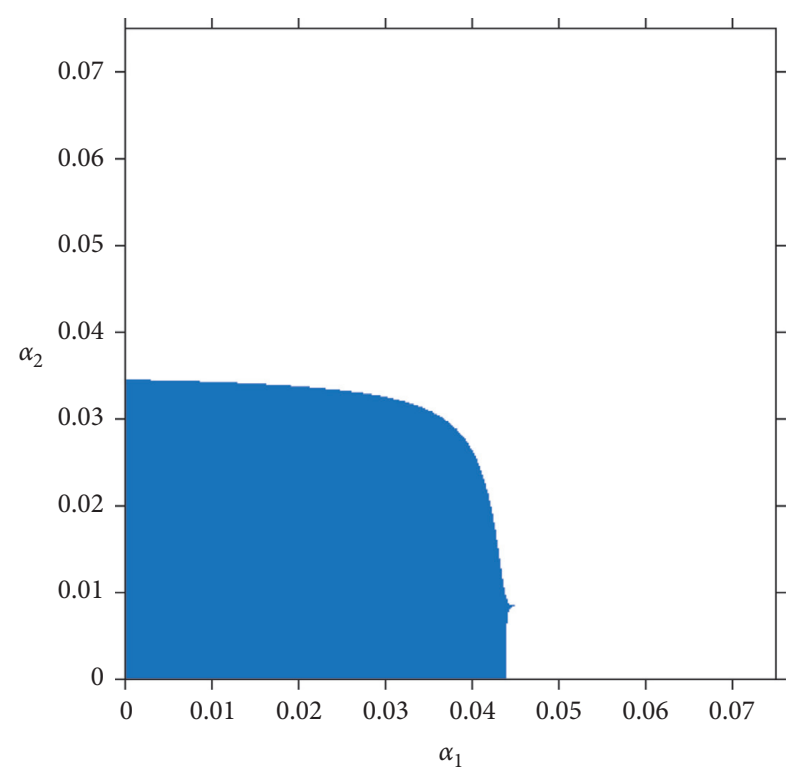

(a)

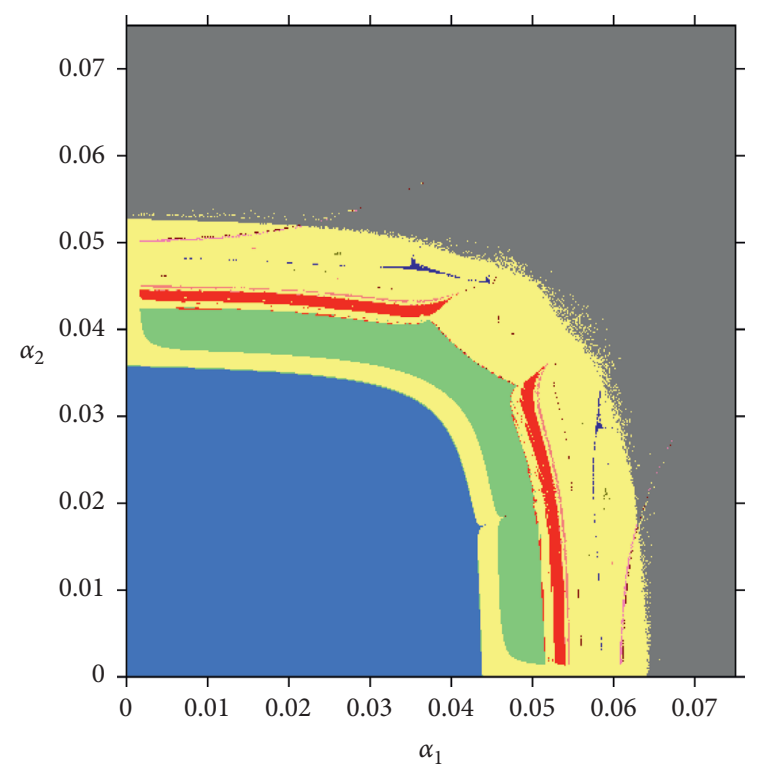

(b)

Figure 2: The stability region diagrams of the system.

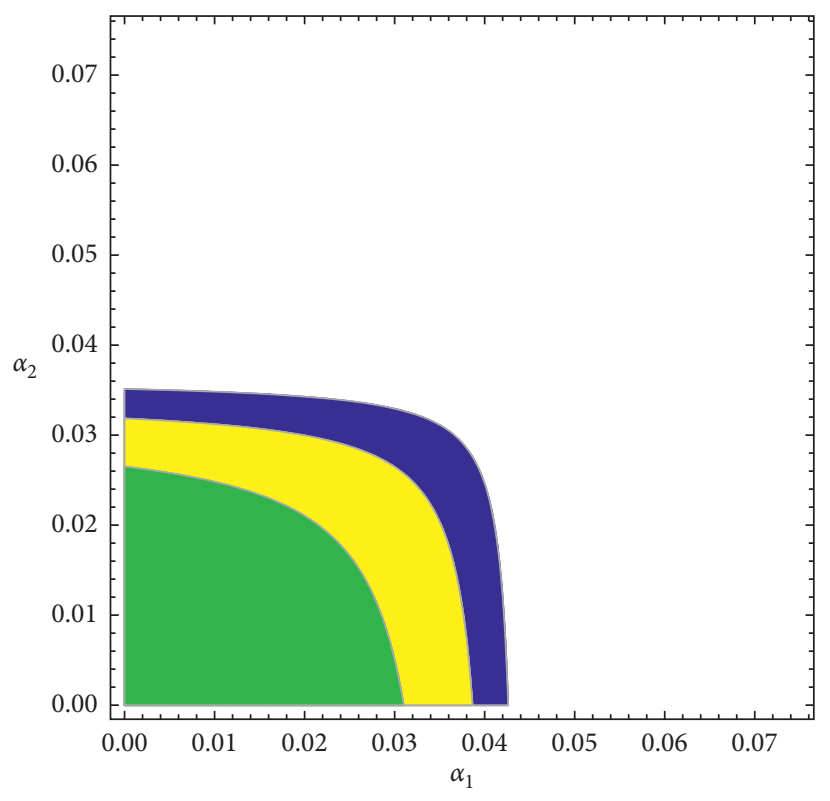

(a)

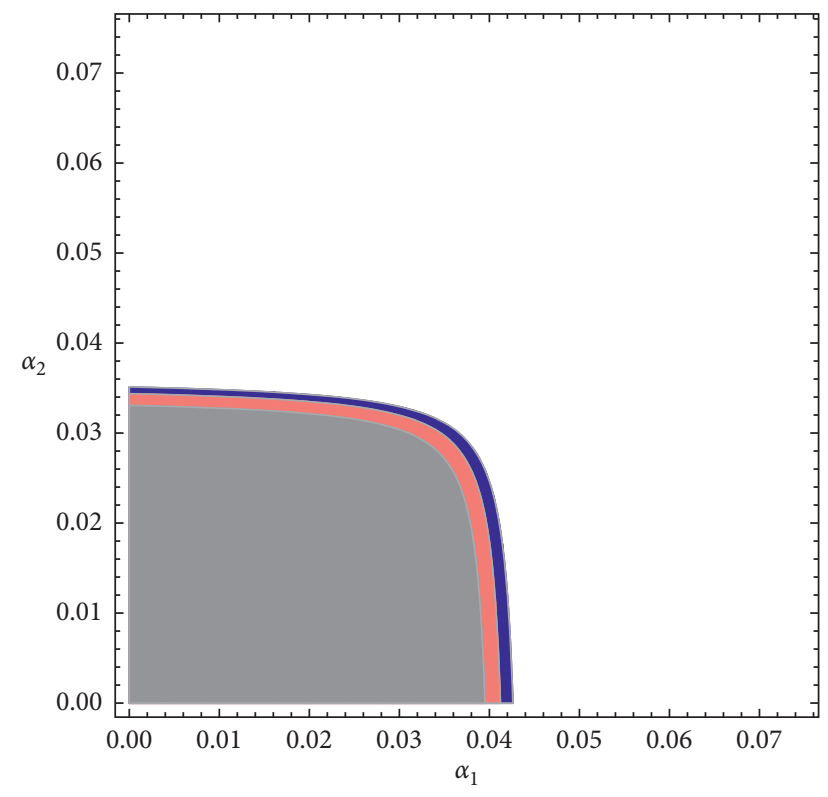

(b)

Figure 3: Continued. 


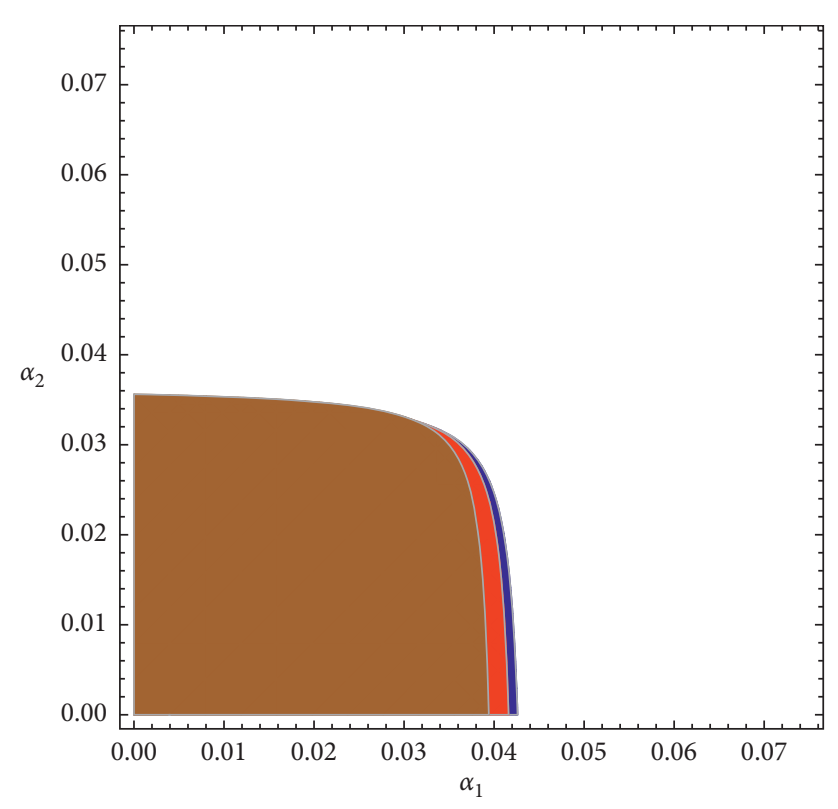

(c)

FIgURE 3: The influence of the parameters $\delta$ and $b_{2}$ on the stable regions.

3.3.3. Complex Dynamics Analysis on the System. Figure 4 gives the bifurcation diagrams, the corresponding largest Lyapunov exponent plot of system (14) and chaos attractor. We assign black curve to wholesale price $w$, blue curve to direct selling price $p_{0}$, red curve to retail price $p_{1}$, green curve to retail price $p_{2}$, and yellow curve to advertising investment B. Figures 4(a) and 4(b) show the bifurcation diagrams of price and the corresponding largest Lyapunov exponent plot of the system with $\alpha_{2}=0.01$ and $\alpha_{1}$ varying from 0 to 0.065 . We can see that with the change of the $\alpha_{1}$, the system enters the chaos from the stable region through flip bifurcation. When $\alpha_{1}=0.044$, the system appears in the first double period orbit. The system becomes chaotic eventually at $\alpha_{1}=0.057$. Figures $4(\mathrm{c})$ and $4(\mathrm{~d})$ give the bifurcation diagrams of price and the corresponding largest Lyapunov exponent plot of the system with $\alpha_{2}$ at $0,0.065$ and $\alpha_{1}$ at 0.01 . We can see that the system appears the first bifurcation at $\alpha_{2}=0.035$ and appears the second bifurcation at $\alpha_{2}=0.043$, and then through a series of bifurcation the system enters into the chaos eventually. Figures $4(\mathrm{e})$ and $4(\mathrm{f})$ show the chaotic attractor when $\alpha_{1}=0.06, \alpha_{2}=0.01$ and $\alpha_{1}=0.01, \alpha_{2}=0.05$. Figures $4(\mathrm{~g})-4(\mathrm{l})$ give the case that the system enters into the chaos through Neimark-Sacker bifurcation. It shows that when $\alpha_{2}=0.038$, with the change of $\alpha_{1}$ the system enters into chaos from the period-two orbit directly. Figures $4(\mathrm{~g})$ and $4(\mathrm{~h})$ show the bifurcation diagram and the corresponding largest Lyapunov exponent plot of the wholesale price $w$ when $\alpha_{2}=0.08$. Figures 4(i)-4(1) show the process from the limit cycle to the strange attractor of the system (18).

3.3.4. The Influence of Parameters on the Profits. Figure 5 shows the influence of adjustment parameters $\alpha_{i}(i=1,2)$ and advertising parameter $\theta$ on the profits of the manufacturer and the retailer. The black curve represents the manufacturer's profits and the blue curve represents the retailer's profits. Figure 5 shows the influence of $\alpha_{i}(i=1,2)$ on the long-term average profit of the supply chain. In stable state, the profits of the manufacturer are more than the profits of the retailer. With the increase in adjustment speed, in Figure 5(a), when $\alpha_{1}>0.0441$, the manufacturer's profits begin to decrease but the retailer's profits begin to increase, and the retailer's profits exceed the manufacturer's profits when $\alpha_{1}>0.0441$. Similarly, Figure 5(b) shows that the profits of the manufacturer decrease while the profits of retailer increase when $\alpha_{1}>0.035$. Therefore, we conclude that, in the dynamic Stackelberg game, the higher adjustment speed of the wholesale or the direct price has adverse effect on the leader manufacturer, but it is favorable for the follower retailer. Figures 5(c) and 5(d) show the influence of advertising parameter $\theta$ on the long-term average profit in the system's stable region $\left(\alpha_{1}=0.01\right.$ and $\left.\alpha_{2}=0.01\right)$ (Figure 5(c)) and bifurcation region $\left(\alpha_{1}=0.01\right.$ and $\alpha_{2}=0.04$ ) (Figure 5(d)), respectively. As shown, with the increase in $\theta$, both in stable region and unstable region, the profit of the manufacturer decreases and the profit of the retailer increases. Therefore, it is unfavorable for the leader manufacturer but favorable for the follower retailer in this model when market is more sensitive to the advertising investment.

\section{Retailer Stackelberg (RS)}

4.1. Model Construction. In this case, the retailer plays the dominant role in the supply chain and becomes the leader of the Stackelberg game. The retailer first decides the retail price $p_{1}$ of the NB product, the retail price $p_{2}$ of the $\mathrm{SB}$ product, and the advertising investment $\mathrm{B}$. The manufacturer decides the wholesale price $w$ and direct selling price $p_{0}$ 


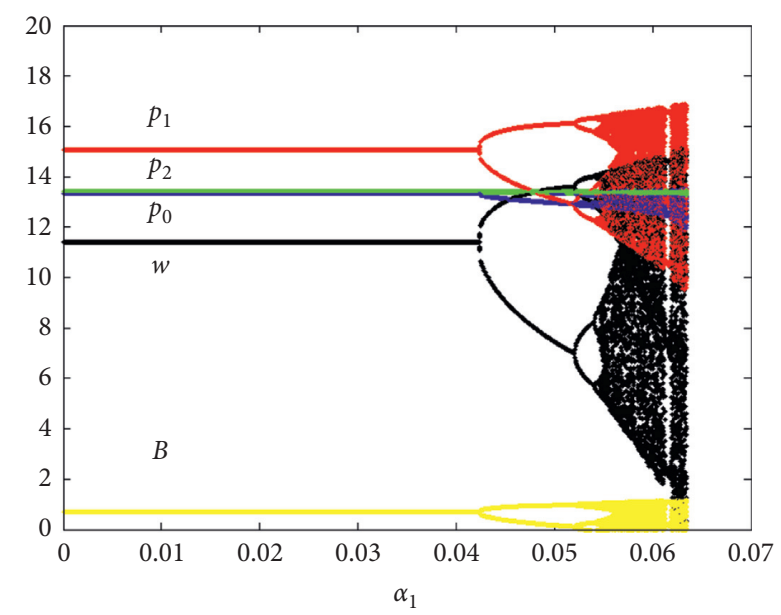

(a)

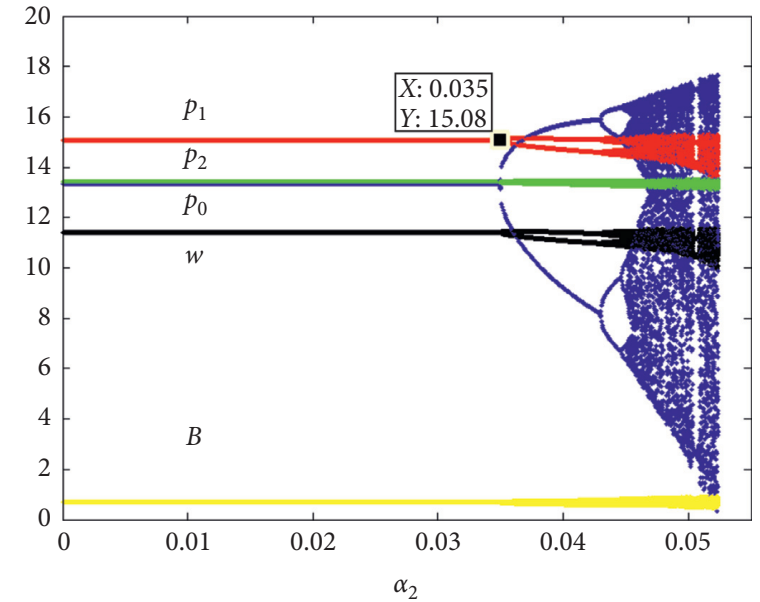

(c)

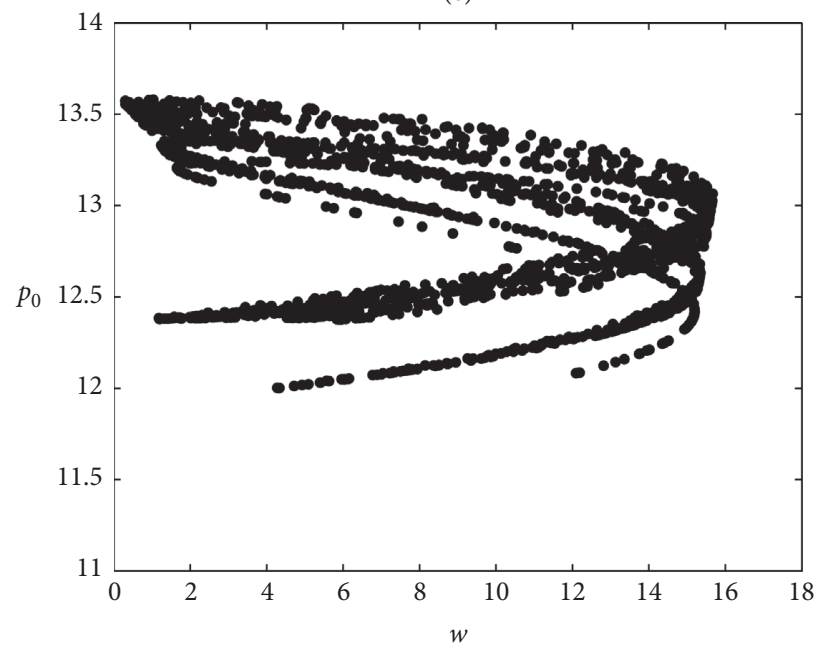

(e)

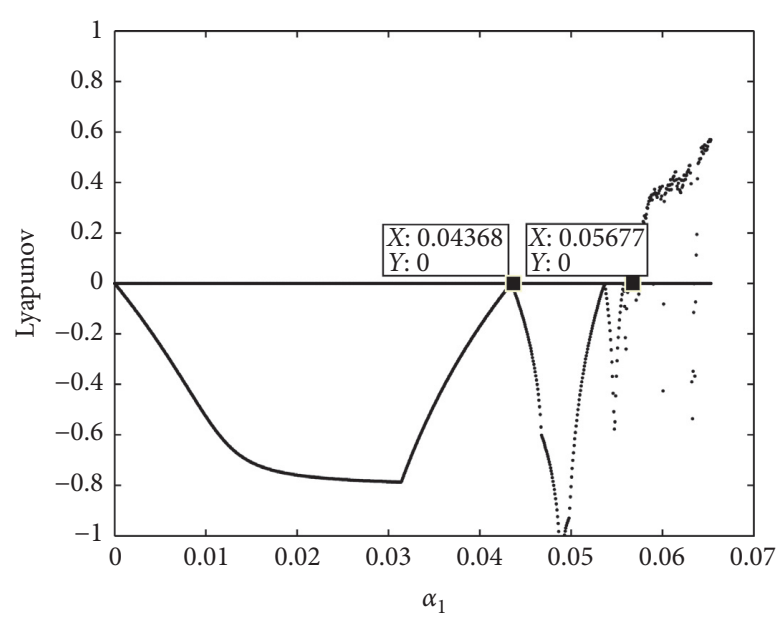

(b)

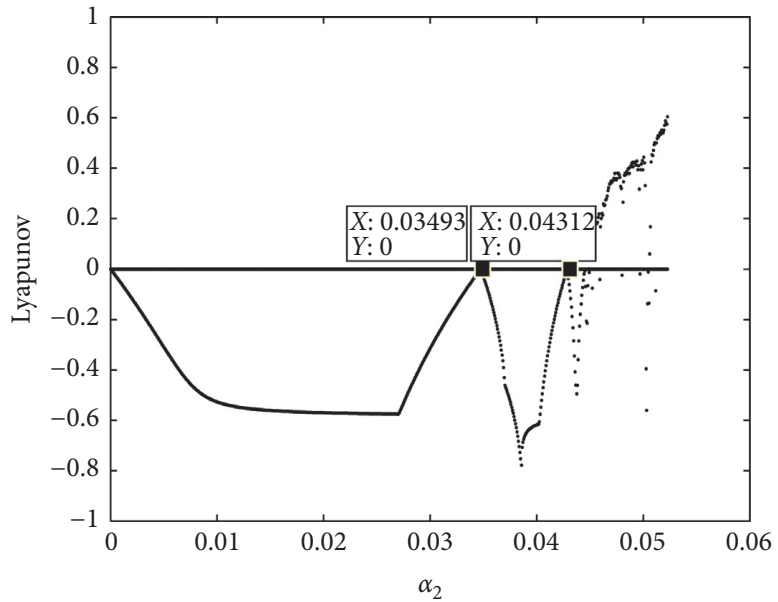

(d)

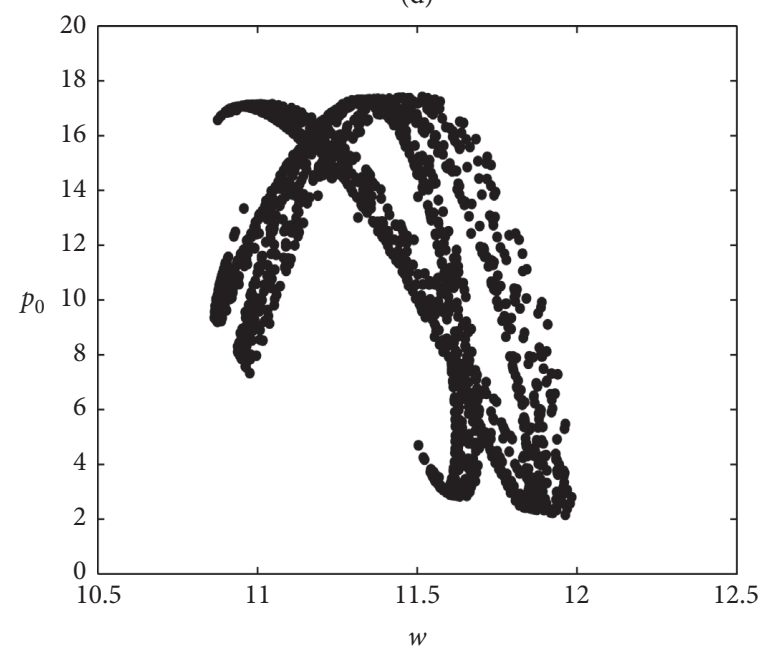

(f)

(a)

FIgURE 4: Continued. 

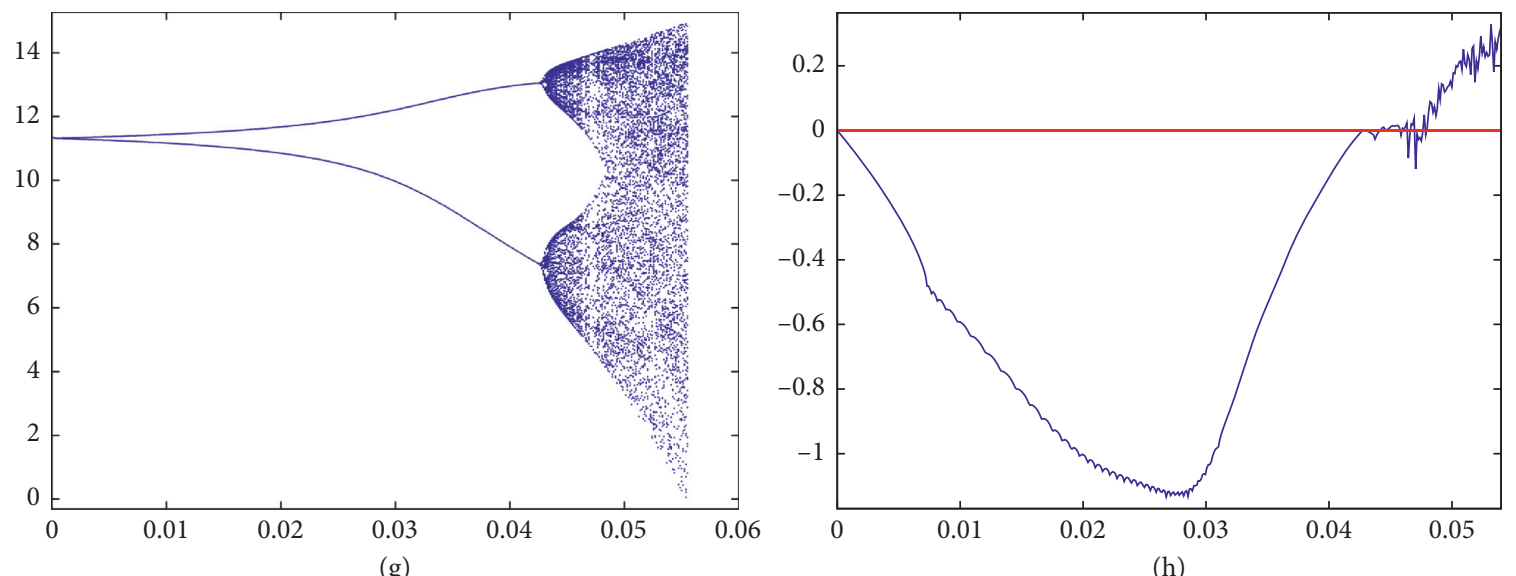

(h)
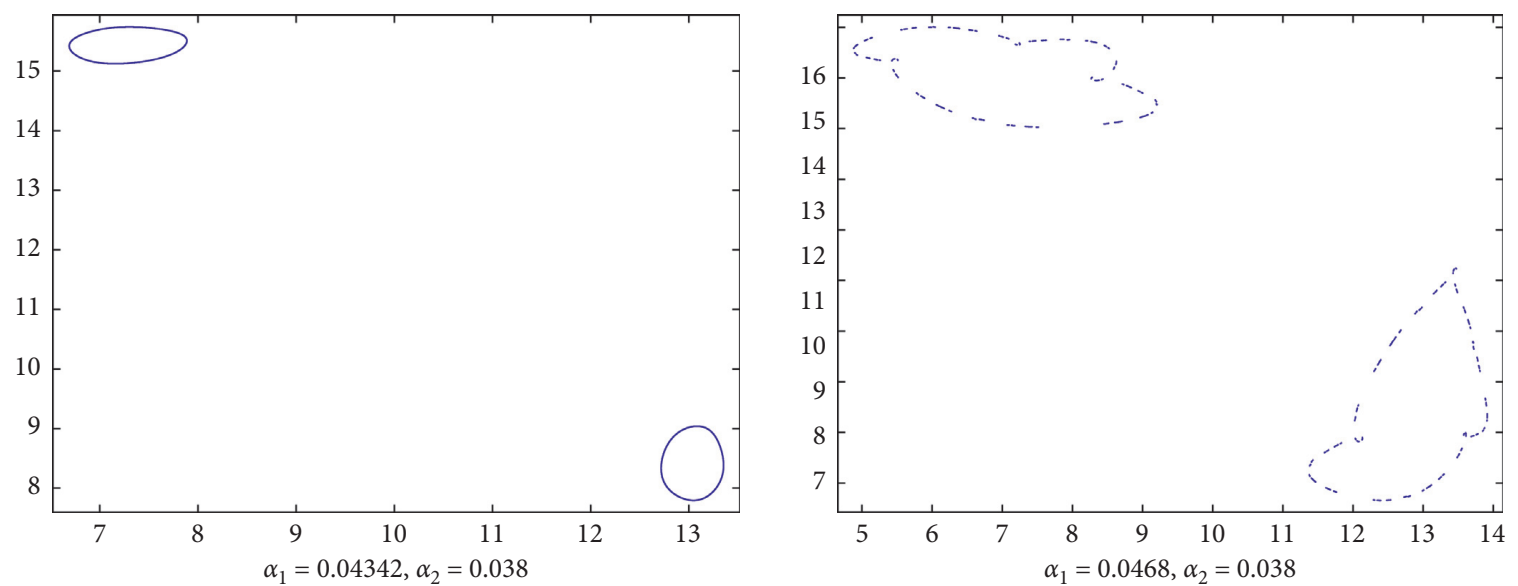

(i)

(j)
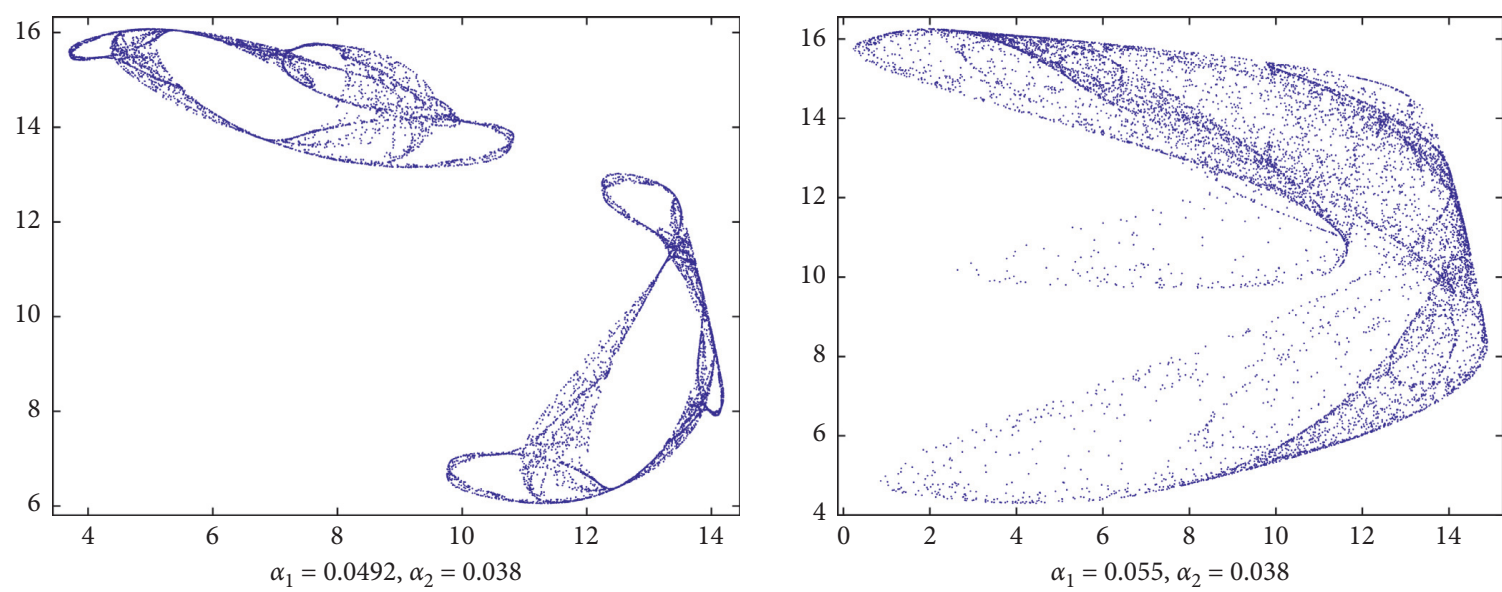

(1)

(b)

FIgURE 4: Bifurcation diagrams and chaos attractor. 


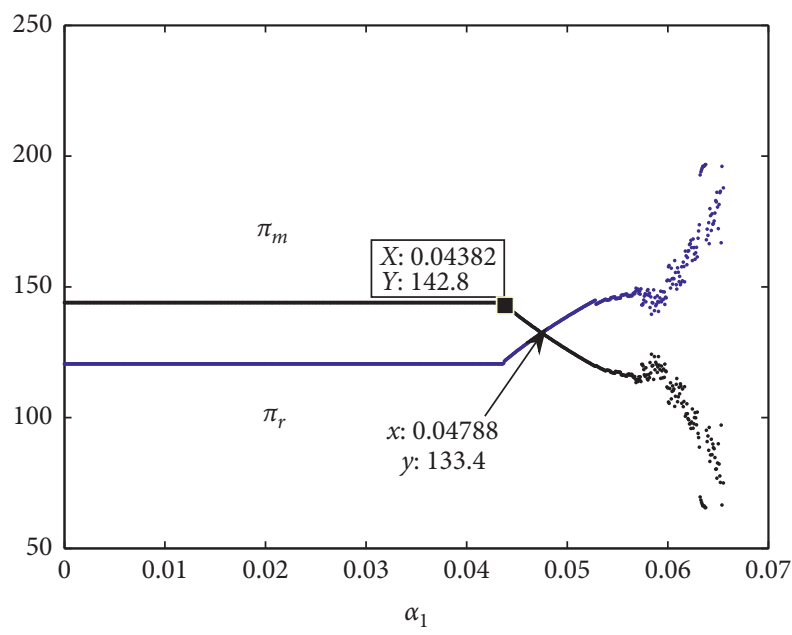

(a)

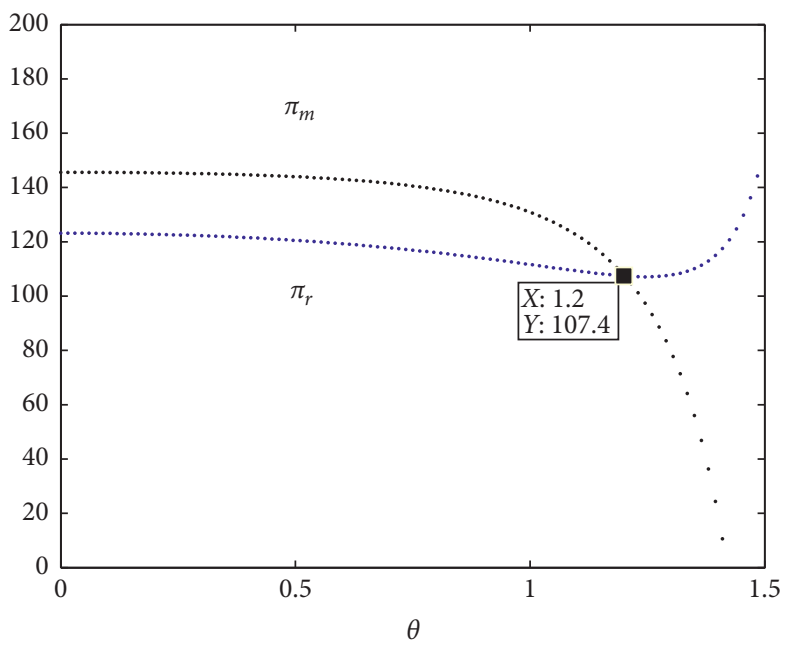

(c)

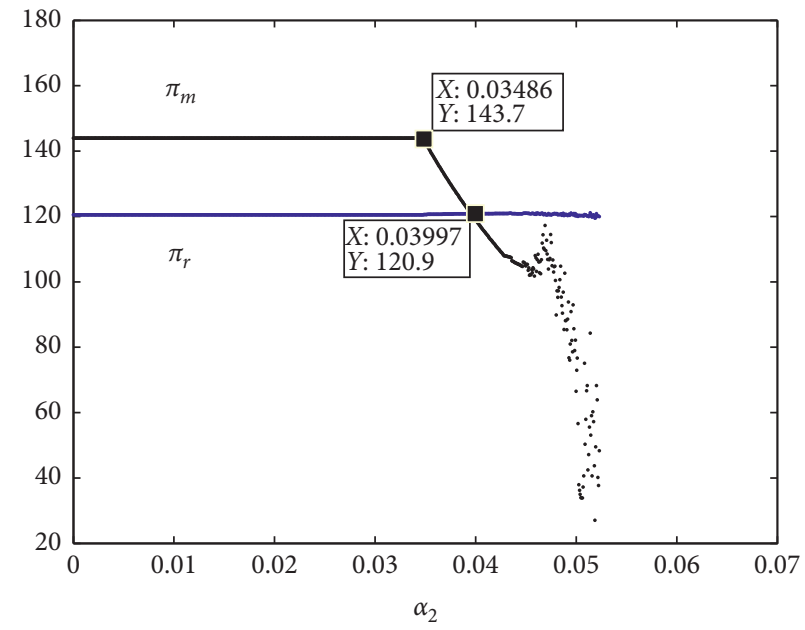

(b)

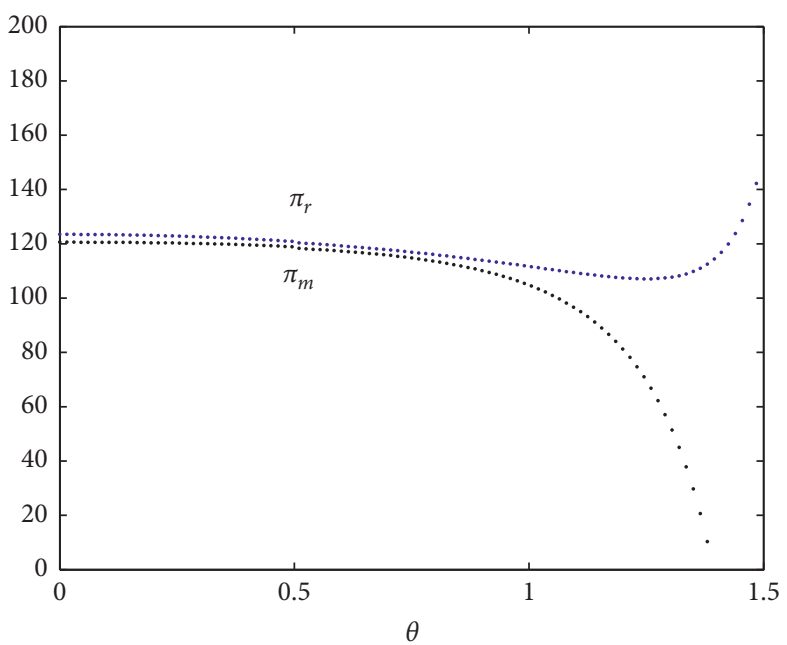

(d)

FIgURE 5: The influence of parameters on the profits.

based on the retailer's decision. We assume the unit retail margin is $u$, and then $p_{1}=w+u$. Similar to the model MS, the optimization problem is solved with backward induction.

The profit function of the manufacturer and the retailer are as follows:

$$
\begin{aligned}
& \pi_{m}^{R}=D_{0}\left(p_{0}-c_{0}\right)+D_{1}\left(w-c_{1}\right), \\
& \pi_{r}^{R}=D_{1} u+D_{2}\left(p_{2}-c_{2}\right)-B^{2} .
\end{aligned}
$$

Taking the first-order partial derivatives of $\pi_{m}^{R}$ with respect to $w$ and $p_{0}$, respectively, and taking the secondorder derivatives further, we can get the Hessian matrix:

$$
\left(\begin{array}{cc}
-2 b_{1} & 2 \gamma \\
2 \gamma & -2 b_{0}
\end{array}\right) .
$$

Note that $-2 b_{1}<0$ and $4 b_{0} b_{1}-4 \gamma^{2}>0$. So, the manufacturer's profit function is concave and has a unique maximum solution. We can work out the manufacturer's best reaction function by $\left(\partial \pi_{m}^{R} / \partial w\right)=0$ and $\left(\partial \pi_{m}^{R} /\right.$ $\left.\partial p_{0}\right)=0$. That is,

$$
\begin{aligned}
w & =\frac{\left(a_{1} b_{0}+b_{0} b_{1} c_{1}-b_{0} b_{1} u+a_{0} \gamma-c_{1} \gamma^{2}+u \gamma^{2}+b_{0} p_{2} \eta-B b_{0} \theta\right)}{2\left(b_{0} b_{1}-\gamma^{2}\right)} \\
& =c_{1}-p_{1}+\frac{a_{1} b_{0}+a_{0} \gamma+b_{0} p_{2} \eta-B b_{0} \theta}{\left(b_{0} b_{1}-\gamma^{2}\right)},
\end{aligned}
$$

$p_{0}=\frac{c_{0}}{2}+\frac{a_{0} b_{1}+a_{1} \gamma+\gamma \eta p_{2}-\gamma \theta B}{2\left(b_{0} b_{1}-\gamma^{2}\right)}$.

Substituting (24) and (25) into (22) and then taking the first-order partial derivatives of $\pi_{r}^{R}$ with respect to $p_{1}, p_{2}$, and $B$, respectively, we can obtain the following 


$$
\begin{aligned}
\frac{\partial \pi_{r}^{R}}{\partial p_{1}}= & a_{1}+b_{1} c_{1}+c_{0} \gamma-c_{1} \eta-4 b_{1} p_{1}+2 \eta p_{2}-B \theta \\
& +\frac{\left(2 a_{1} b_{0} b_{1}+2 a_{0} b_{1} \gamma+2 b_{0} b_{1}\left(\eta p_{2}-B \theta\right)\right)}{\left(b_{0} b_{1}-\gamma^{2}\right)} \\
\frac{\partial \pi_{r}^{R}}{\partial p_{2}}= & a_{2}+b_{2} c_{2}-\frac{c_{1} \eta}{2}-2 b_{2} p_{2}+2 \eta p_{1}+\theta B \\
& +\frac{\left(b_{0}^{2} b_{1} \eta\left(-\eta p_{2}+\theta B\right)-b_{0} b_{1} \eta\left(a_{0} \gamma+a_{1} b_{0}\right)\right)}{\left(b_{0} b_{1}-\gamma^{2}\right)^{2}} \\
& +\frac{\eta\left(a_{0} \gamma+b_{0} b_{1} c_{1}+2 a_{1} b_{0}+b_{0} c_{0} \gamma\right)}{2\left(-b_{0} b_{1}+\gamma^{2}\right)} \\
& +\frac{2 b_{0} b_{1} \eta p_{1}-b_{0} \eta^{2} p_{2}+b_{0} \eta \theta B}{b_{0} b_{1}-\gamma^{2}}, \\
\frac{\partial \pi_{r}^{R}}{\partial B}= & \frac{1}{2\left(-b_{0} b_{1}+\gamma^{2}\right)^{2}}\left(\left(\gamma^{3}\left(-a_{0}+\left(c_{1}-2\left(c_{2}+p_{1}-p_{2}\right)\right) \gamma\right)\right.\right. \\
& +2 a_{1} b_{0}\left(2 b_{0} b_{1}-\gamma^{2}\right)+b_{0}^{2} b_{1}\left(2 b_{1}\left(c_{1}-c_{2}-3 p_{1}+p_{2}\right)\right. \\
& \left.+c_{0} \gamma+4 p_{2} \eta\right)-b_{0} \gamma\left(-3 a_{0} b_{1}+\gamma\left(b_{1}\right.\right. \\
& \left.\left.\left.\cdot\left(3 c_{1}-4 c_{2}-8 p_{1}+4 p_{2}\right)+c_{0} \gamma+2 p_{2} \eta\right)\right)\right) \theta+B \\
& \left.\cdot\left(-4 \gamma^{4}-4 b_{0}^{2} b_{1}\left(b_{1}+\theta^{2}\right)+2 b_{0} \gamma^{2}\left(4 b_{1}+\theta^{2}\right)\right)\right) .
\end{aligned}
$$

Taking the second-order derivatives further, we can get the Hessian matrix:

$$
\left(\begin{array}{ccc}
-4 b_{1} & f_{1} & f_{2} \\
f_{1} & f_{3} & f_{4} \\
f_{2} & f_{4} & f_{5}
\end{array}\right),
$$

where

$$
\begin{aligned}
& f_{1}=\frac{4 b_{0} b_{1} \eta-2 \gamma^{2} \eta}{b_{0} b_{1}-\gamma^{2}}, \\
& f_{2}=\frac{\left(-3 b_{0} b_{1}+\gamma^{2}\right) \theta}{b_{0} b_{1}-\gamma^{2}}, \\
& f_{3}=-2 b_{2}+\frac{b_{0}\left(-2 b_{0} b_{1}+\gamma^{2}\right) \eta^{2}}{\left(-b_{0} b_{1}+\gamma^{2}\right)^{2}}, \\
& f_{4}=\frac{\left(\gamma^{4}-b_{0} \gamma^{2}\left(2 b_{1}+\eta\right)+b_{0}^{2} b_{1}\left(b_{1}+2 \eta\right)\right) \theta}{\left(-b_{0} b_{1}+\gamma^{2}\right)^{2}}, \\
& f_{5}=\frac{-2 \gamma^{4}-2 b_{0}^{2} b_{1}\left(b_{1}+\theta^{2}\right)+b_{0} \gamma^{2}\left(4 b_{1}+\theta^{2}\right)}{\left(-b_{0} b_{1}+\gamma^{2}\right)^{2}} .
\end{aligned}
$$

While satisfying

$$
\begin{aligned}
& \frac{8 b_{0} b_{1}\left(b_{1} b_{2}-\eta^{2}\right)+4 \gamma^{2}\left(-2 b_{1} b_{2}+\eta^{2}\right)}{b_{0} b_{1}-\gamma^{2}}>0, \\
& \frac{1}{\left(-b_{0} b_{1}+\gamma^{2}\right)^{2}}\left(2 \gamma^{4}\left(-8 b_{1} b_{2}+4 \eta^{2}+2 b_{1} \theta^{2}+b_{2} \theta^{2}-2 \eta \theta^{2}\right)\right. \\
& +2 b_{0}^{2} b_{1}\left(\eta^{2} \theta^{2}+b_{1}^{2}\left(-8 b_{2}+2 \theta^{2}\right)+b_{1}\left(8 \eta^{2}+b_{2} \theta^{2}-4 \eta \theta^{2}\right)\right) \\
& \left.+b_{0} \gamma^{2}\left(-\eta^{2} \theta^{2}+8 b_{1}^{2}\left(4 b_{2}-\theta^{2}\right)-4 b_{1}\left(6 \eta^{2}+b_{2} \theta^{2}-3 \eta \theta^{2}\right)\right)\right)<0,
\end{aligned}
$$

the retailer's profit function is concave and has a unique maximum solution because the Hessian matrix is negative definite. So, we get the optimal retail price $p_{1}^{R}, p_{2}^{R}$, and $B^{R}$. Furthermore, we also get the optimal wholesale price $w^{R}$ and direct selling price $p_{0}^{R}$ (see Appendix A.16-A.20).

Similar to the MS model, we develop a dynamic Stackelberg game model. Suppose that the decision in next period can be adjusted with bounded rationality and is based on partial estimation of the marginal profits of the current period. The model can be constructed as follows:

$$
\left\{\begin{array}{l}
p_{1}(t+1)=p_{1}(t)+\beta_{1} p_{1}(t) \frac{\partial \pi_{r}^{R}\left(p_{1}, p_{2}, B\right)}{\partial p_{1}(t)}, \\
p_{2}(t+1)=p_{2}(t)+\beta_{2} p_{2}(t) \frac{\partial \pi_{r}^{R}\left(p_{1}, p_{2}, B\right)}{\partial p_{2}(t)}, \\
B(t+1)=B(t)+\beta_{3} B(t) \frac{\partial \pi_{r}^{R}\left(p_{1}, p_{2}, B\right)}{\partial B(t)} .
\end{array}\right.
$$

System (45) gives the retailer's dynamic price decision, and the decision variables directly relate to the positive parameter $\beta_{i}(i=1,2,3)$ which represents the price adjustment speed. $\left(\partial \pi_{R}^{R}\left(p_{1}, p_{2}, B\right)\right) /\left(\partial p_{1}(t)\right), \quad\left(\partial \pi_{R}^{R}\left(p_{1}, p_{2}, B\right)\right) /$ $\left(\partial p_{2}(t)\right)$, and $\left(\partial \pi_{R}^{R}\left(p_{1}, p_{2}, B\right)\right) /(\partial B(t))$ are the marginal profits, and they can be obtained from (26)-(28). Furthermore, the manufacturer's price decision can be worked out based on $p_{1}(t), p_{2}(t)$, and $B(t)$.

$$
\begin{aligned}
& w(t)=c_{1}-p_{1}(t)+\frac{a_{1} b_{0}+a_{0} \gamma+b_{0} \eta p_{2}(t)-b_{0} \theta B(t)}{\left(b_{0} b_{1}-\gamma^{2}\right)}, \\
& p_{0}(t)=\frac{c_{0}}{2}+\frac{a_{0} b_{1}+a_{1} \gamma+\gamma \eta p_{2}(t)-\gamma \theta B(t)}{2\left(b_{0} b_{1}-\gamma^{2}\right)} .
\end{aligned}
$$

4.2. Model Analysis. The eight equilibrium solutions of system (33) $\left(E_{1}, E_{2}, \ldots, E_{8}\right)$ can be shown as follows (here only giving the nonzero equilibrium solutions): 


$$
\begin{aligned}
E_{7}= & \left\{\left(4 a_{1}\left(3 b_{0}^{2} b_{1}^{2}-4 b_{0} b_{1} \gamma^{2}+\gamma^{4}\right)+\gamma^{3}\left(a_{0}\left(-8 b_{1}+\theta^{2}\right)+\gamma\left(4 b_{1} c_{1}+4 c_{0} \gamma-4 c_{2} \eta-c_{1} \theta^{2}+2 c_{2} \theta^{2}\right)\right)\right.\right. \\
& +b_{0}^{2} b_{1}\left(4 b_{1}^{2} c_{1}+\left(c_{0} \gamma-4 c_{2} \eta\right) \theta^{2}+b_{1}\left(4 c_{0} \gamma-4 c_{2} \eta-2 c_{1} \theta^{2}+6 c_{2} \theta^{2}\right)\right)-b_{0} \gamma\left(a_{0} b_{1}\left(-8 b_{1}+\theta^{2}\right)\right. \\
& \left.\left.+\gamma\left(8 b_{1}^{2} c_{1}+\left(c_{0} \gamma-2 c_{2} \eta\right) \theta^{2} 2+b_{1}\left(8 c_{0} \gamma-8 c_{2} \eta-3 c_{1} \theta^{2}+8 c_{2} \theta^{2}\right)\right)\right)\right) \\
& \cdot\left(2\left(-b_{0} b_{1}+\gamma^{2}\right)^{2}\left(8 b_{1}-\theta^{2}\right)\right)^{-1}, 0,\left(\left(a_{1}\left(-b_{0} b_{1}+\gamma^{2}\right)+\gamma^{2}\left(-b_{1} c_{1}+4 b_{1} c_{2}+c_{0} \gamma-c_{2} \eta\right)\right.\right. \\
& \left.\left.\left.+b_{0} b_{1}\left(b_{1} c_{1}-4 b_{1} c_{2}-c_{0} \gamma+3 c_{2} \eta\right)\right) \theta\right) \cdot\left(\left(b_{0} b_{1}-\gamma^{2}\right)\left(8 b_{1}-\theta^{2}\right)\right)^{-1}\right\}, \\
E^{R}= & \left(p_{1}^{R}, p_{2}^{R}, B^{R}\right) .
\end{aligned}
$$

$E_{i}(i=1,2, \ldots, 7)$ are boundary equilibrium solutions, while $E^{R}$ is the only Stackelberg equilibrium solution. Its expression is shown (A.16)-(A.18) in Appendix. In order to guarantee the practical significance, we assume that all equilibriums are positive.

The Jacobian matrix of system (33) is given by

$$
\left(\begin{array}{ccc}
1+\beta_{1} t_{1} & \beta_{2} f_{1} p_{2} & \beta_{3} f_{2} B \\
\beta_{1} f_{1} p_{1} & 1+\beta_{2} t_{2} & \beta_{3} f_{4} B \\
\beta_{1} f_{2} p_{1} & \beta_{2} f_{4} p_{2} & 1+\beta_{3} t_{3}
\end{array}\right),
$$

where

$$
\begin{aligned}
t_{1}= & a_{1}+b_{1} c_{1}-\eta c_{2}+\gamma c_{0}+2 \eta p_{2}-8 b_{1} p_{1}-B \theta \\
& +\frac{2 b_{1}\left(a_{1} b_{0}+a_{0} \gamma+b_{0} \eta p_{2}-b_{0} B \theta\right)}{b_{0} b_{1}-\gamma^{2}}, \\
t_{2}= & \frac{1}{2\left(-b_{0} b_{1}+\gamma^{2}\right)^{2}}\left(2 a_{2}\left(-b_{0} b_{1}+\gamma^{2}\right)^{2}+\gamma^{3}\left(2 b_{2}\left(c_{2}-4 p_{2}\right) \gamma\right.\right. \\
& \left.+a_{0} \eta-c_{1} \gamma \eta+4 p_{1} \gamma \eta+2 B \gamma \theta\right)+b_{0}^{2} b_{1}\left(-\eta\left(4 a_{1}+c_{0} \gamma\right.\right. \\
& \left.\left.+8 p_{2} \eta-4 B \theta\right)+2 b_{1}\left(b_{2} c_{2}-4 b_{2} p_{2}-c_{1} \eta+4 p_{1} \eta+B \theta\right)\right) \\
& +b_{0} \gamma\left(\gamma \eta\left(2 a_{1}+c_{0} \gamma+4 p_{2} \eta-2 B \theta\right)-b_{1}\left(4 b_{2}\left(c_{2}-4 p_{2}\right) \gamma\right.\right. \\
& \left.\left.\left.+3 a_{0} \eta-3 c_{1} \gamma \eta+12 p_{1} \gamma \eta+4 B \gamma \theta\right)\right)\right) \\
t_{3}= & \frac{1}{2\left(-b_{0} b_{1}+\gamma^{2}\right)^{2}}\left(\left(\gamma^{3}\left(-a_{0}+\left(c_{1}-2\left(c_{2}+p_{1}-p_{2}\right)\right) \gamma\right)\right.\right. \\
& +2 a_{1} b_{0}\left(2 b_{0} b_{1}-\gamma^{2}\right)+b_{0}^{2} b_{1}\left(2 b_{1}\left(c_{1}-c_{2}-3 p_{1}+p_{2}\right)\right. \\
& \left.+c_{0} \gamma+4 p_{2} \eta\right)-b_{0} \gamma\left(-3 a_{0} b_{1}+\gamma\left(b _ { 1 } \left(3 c_{1}-4 c_{2}-8 p_{1}\right.\right.\right. \\
& \left.\left.\left.\left.+4 p_{2}\right)+c_{0} \gamma+2 p_{2} \eta\right)\right)\right) \theta-4 B\left(2 \gamma^{4}+2 b_{0}^{2} b_{1}\left(b_{1}+\theta^{2}\right)\right. \\
& \left.\left.\left.+4 b_{1}+\theta^{2}\right)\right)\right) . \\
& \left.+b_{0}\right) \\
& \\
& \\
&
\end{aligned}
$$

The stability of equilibrium points will be determined by the nature of the eigenvalues of the Jacobian matrix evaluated at the corresponding equilibrium points. Then, we substitute the value of $E_{i}(i=1,2 \ldots, 7)$ into (36) and we have the following proposition.

Proposition 2. All the boundary equilibrium points $E_{i}(i=$ $1,2 \ldots, 7)$ are unstable under our assumptions and conditions (31) and (32).

We investigate the stability of $E^{M}$ by utilizing Jury's stability condition [22]. Assuming the characteristic polynomial for the Jacobian matrix $J$ is

$$
f(\lambda)=\lambda^{3}+A \lambda^{2}+B \lambda+C .
$$

Then, the local stability domain can be determined by the following conditions:

$$
\begin{aligned}
& \text { (i) : } f(1)=A+B+C+1>0, \\
& \text { (ii) : } f(-1)=A-B+C-1<0, \\
& \text { (iii) : } C^{2}-1<0, \\
& \text { (iv) : }\left(1-C^{2}\right)^{2}-(B-A C)^{2}>0 .
\end{aligned}
$$

4.3. The Experimental Calculation and Analysis. In this section, numerical simulations are carried to show the influence of parameters. We will study how the adjust parameters, advertising coefficients, and cost parameters affect the product prices and the long-term average profit. We first choose some values for some parameters based on actual competition: $a_{0}=30 ; a_{1}=55 ; a_{2}=35 ; b_{0}=2.2 ; b_{1}=4.05$; $b_{2}=2.35 ; \gamma=0.7 ; \eta=0.6 ; \theta=0.5 ; c_{0}=7 ; c_{1}=5 ;$ and $c_{2}=7$ (the values are the same as the model of MS). These parameters should meet the model assumptions and conditions (34) and (A.16). We can calculate the equilibrium prices of the system: $p_{1}^{R}=15.0997 ; \quad p_{2}^{R}=13.4153$; $B^{R}=0.6879 ; w^{R}=8.7782 ;$ and $p_{0}^{R}=13.3215$. The manufacturer and retailer's optimal profit of stable region are $\pi_{m}^{R}=112.289$ and $\pi_{r}^{R}=150.898$.

4.3.1. The Stability Region and the Bifurcation Diagrams of the System. In Figure 6, the stability of the equilibrium region is presented. We can see that the system is stable when $\beta_{1}<0.008, \beta_{2}<0.02$, and $\beta_{3}<1.4$. A high adjustment speed will make the system leave the stable area. 


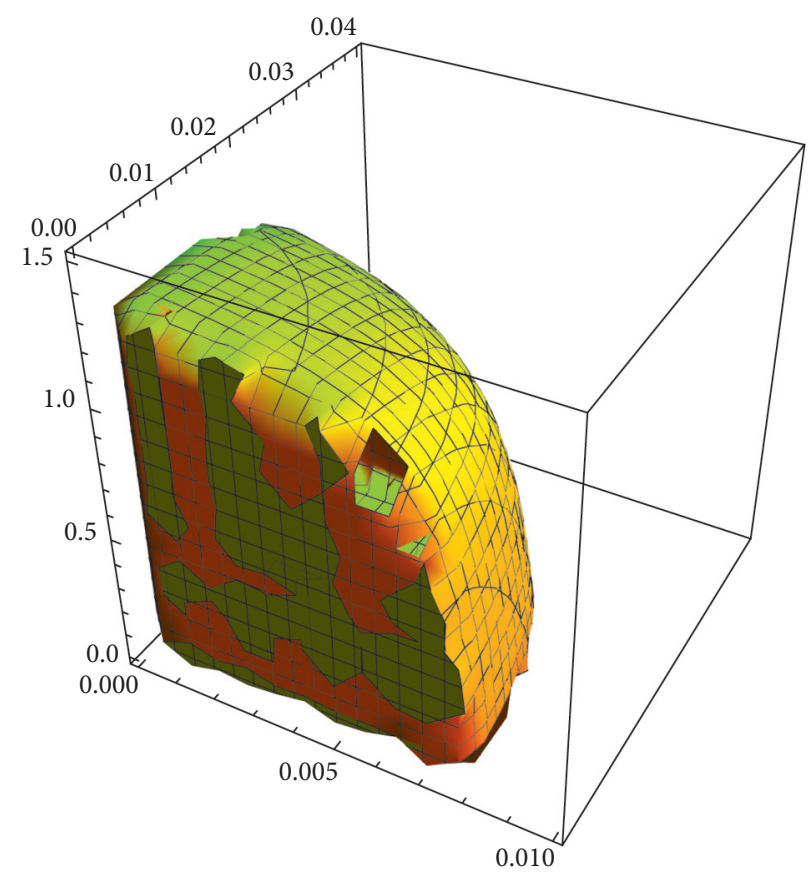

FIGURE 6: The stability region of the system.

Figure 7 (a) presents the parameter basin with respect to the parameters $\left(\beta_{1}, \beta_{2}\right)\left(\beta_{3}=0.1\right)$ and assigns different colors to different periods (see the MS model). From this two-dimensional bifurcation diagram of the system, we can see that the system enters into chaos through period-doubling bifurcation and Neimark-Sacker bifurcation. Figure 7 (b) gives the bifurcation diagrams of the retail price $p_{1}$ with $\beta_{2}=0.018, \beta_{3}=0.1$, and $\beta_{1}$ at $0,0.012$. Figure $7(\mathrm{c})$ gives the limit cycle diagram when $\beta_{1}=0.0103, \beta_{2}=$ 0.018 , and $\beta_{3}=0.1$. Figure $7(\mathrm{~d})$ is the corresponding largest Lyapunov exponent plot.

4.3.2. Chaos Attractor and Sensitivity to Initial Values. Figure 8 shows the chaotic attractor and the butterfly effect. Figure $8(\mathrm{a})$ is the chaotic attractor of system (33) when $\beta_{1}=0.011, \beta_{2}=0.01$, and $\beta_{3}=0.1$. Figures $8(b)-8(d)$ show the sensitivity to initial values of system (33) when $p_{1}=15.09969, p_{2}=13.4153$, and $B=0.68792$. In Figure $8(\mathrm{~b})$, blue curve is the retail price $p_{1}$ with the initial values $p_{1}=15.09969$, and red curve is the retail price $p_{1}$ with the initial values $p_{1}=15.09968$. We can see from the figure that when the retail price $p_{1}$ initial value changed 0.00001 , the system tends to be significantly different after about 20 cycles iteration. Similarly, Figures $8(\mathrm{c})-8(\mathrm{~d})$ give the case when the retail price $p_{2}$ initial value changed 0.0001 and the advertising investment $B$ changed 0.00001 . It can be seen that a small change of the initial value will cause quite different results in the end.

4.3.3. The Influence of Parameters on the Profits. Figures $9(\mathrm{a})-9(\mathrm{~d})$ give the influence of $\beta_{i}(i=1,2,3)$ on the longterm average profit of the manufacturer (black curve) and the retailer (blue curve). As shown, with the increase of the $\beta_{i}(i=1,2,3)$, the profit of the manufacturer and the retailer is stable at first, and when the system enters into the bifurcation region, the retailer's profits begin to decrease. But, the result is opposite for the manufacturer. Therefore, we conclude that in the RS model, the higher adjustment speed of the price is adverse to the leader retailer, but it is favorable to the follower manufacturer.

Figures 9(e)-9(j) show the influence of the cost $c_{0}$, channel competition coefficient $\gamma$, and brand competition coefficient $\eta$ on the long-term average profit in the system stable region $\left(\beta_{1}=0.001, \beta_{2}=0.01\right.$, and $\left.\beta_{3}=0.1\right)$ and the bifurcation region $\left(\beta_{1}=0.01, \beta_{2}=0.01\right.$, and $\left.\beta_{3}=0.1\right)$, respectively. From Figures 9(e) and 9(f), we can see that both profits are in the stable region and the bifurcation region separately. So, in the RS model, the manufacturers can improve their profit through controlling the cost of the direct channel. From Figures 9(g) and 9(h), we can see that channel competition coefficient $\gamma$ has obvious effects on profits. In the stable region, with the increase of $\gamma$, both manufacturer's profit and retailer's profit are rising; the profit of the manufacturer rise significantly greater than that of the retailer; when $\gamma>0.005$, the profit of the manufacturer and the profit of the retailer are the same; when $\gamma>0.005$, the profit of the manufacturer exceed the profit of the retailer. In the bifurcation region with the increase of $\gamma$, the manufacturer's profit is still increasing while the retailer's profit decreases rapidly. It shows that in the RS model, the manufacturer who sets up a direct sales channel is more advantageous than the retailer when the market is sensitive to channel competition. Figures 9(i) and 9(j) give the influence of the brand competition coefficient $\eta$ on the profit. We can see from the figure, in the stable region, with the increase of $\eta$, both manufacturer's and retailer's profit will increase, but when the system enters into the bifurcation 


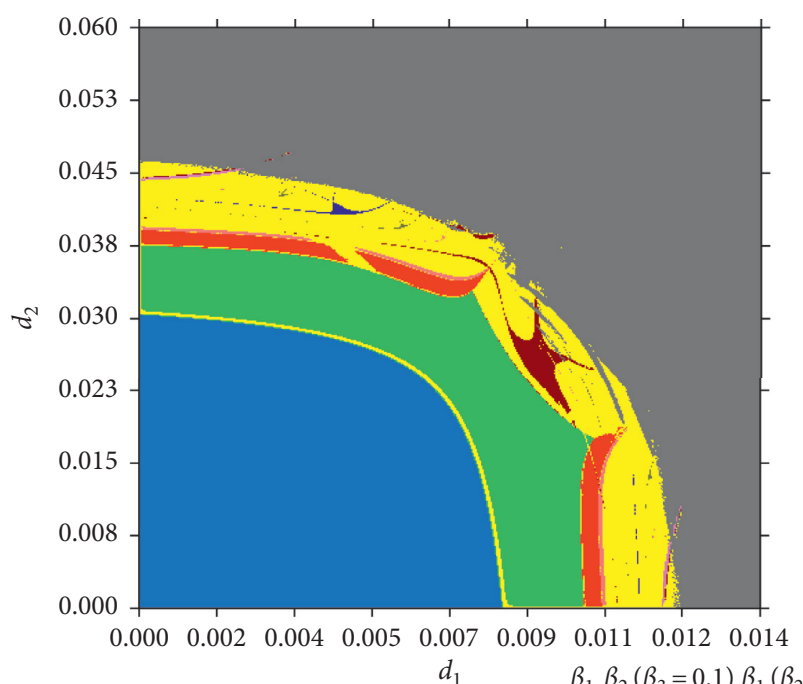

(a)

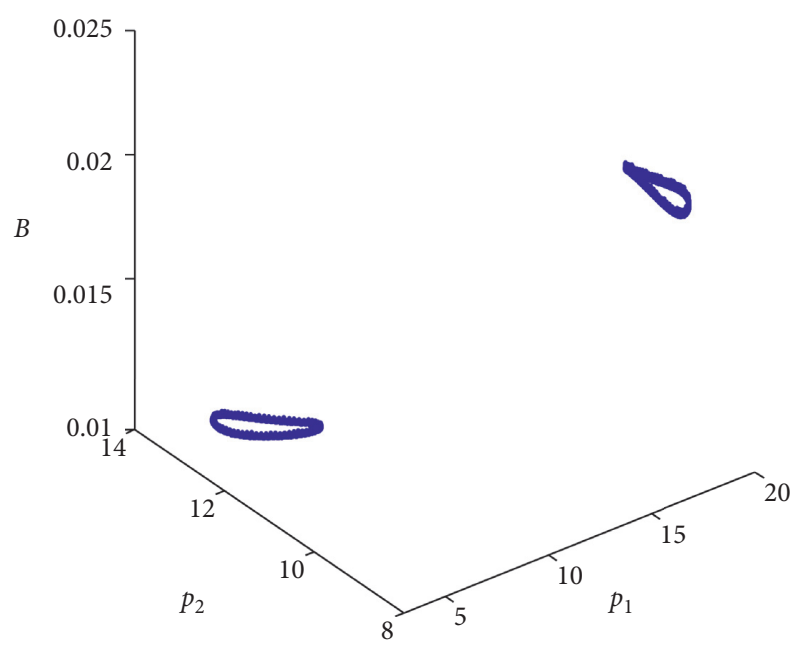

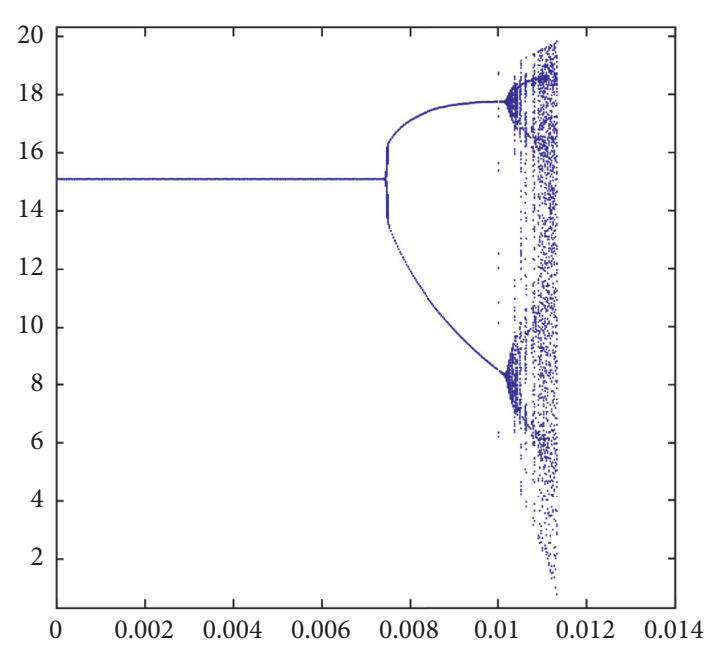

(b)

$$
\beta_{1}=0.0103, \beta_{2}=0.018, \beta_{3}=0.1 \beta_{1}\left(\beta_{2}=0.018, \beta_{3}=0.1\right)
$$

(c)

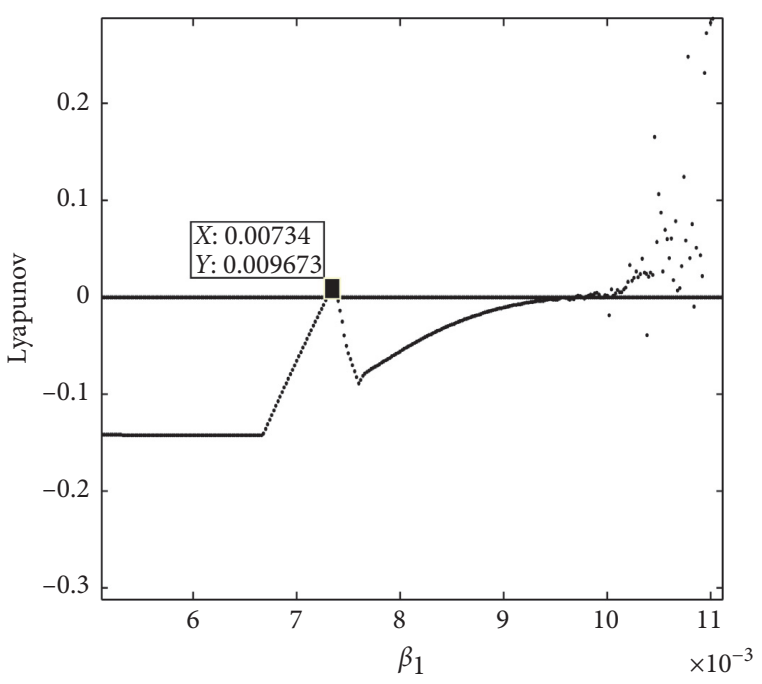

(d)

Figure 7: Two-dimensional bifurcation diagrams of the system.

region, the manufacturer's profit is still increasing while the retailer's profit decreases rapidly. So, in a word, controlling the cost and increasing the channel competitive strength and brand competition strength will make the manufacturer increase its profit in the game dominated by the retailer.

\section{Comparison of the Two Models and Analysis}

In this section, we compare the prices and profits of the two models in the stable region and in the bifurcation region, respectively. The results are shown in Table 1.

Table 1 shows the prices and the profits of the two models in different regions.

In Table 1, we compare different variables of the two models in the stable region and bifurcation region, respectively. It can be seen from Table 1, in the stable region, the wholesale price and direct selling price is higher in the MS model than that in the RS model. The total profit of the supply chain in the MS model is also more than that in the RS model. In the RS model, the lower wholesale price makes the retailer gain more profit. In the bifurcation region, with the increase of the competitive level, both in the MS model and the RS model, the retailer has the lower NB product price and SB product price. In addition, we can see that the game leader will have advantages in the stability region, but once enters into the bifurcation region or the chaos region, the game followers will adjust their price to follow the leader's decision so that they can make a profit.

\section{Conclusions}

This paper researches two noncooperative dynamic pricing strategies used in a supply chain. We establish dynamic Stackelberg game models in which either the manufacturer or the retailer acts as a leader. In these two models, the manufacturer sells a national-brand (NB) product to an 


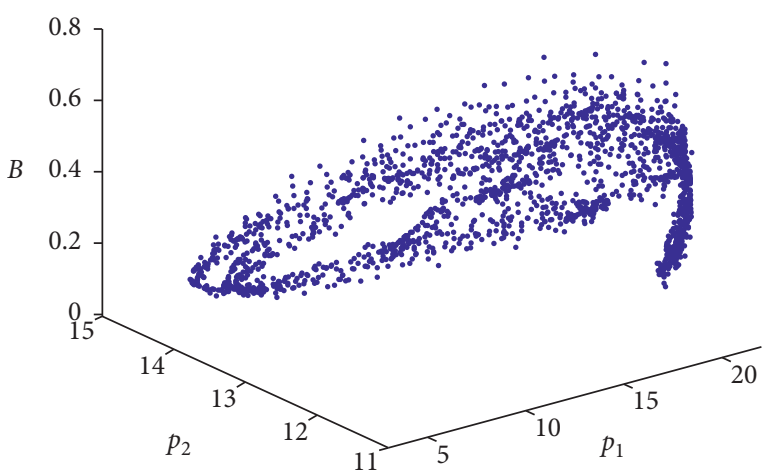

(a)

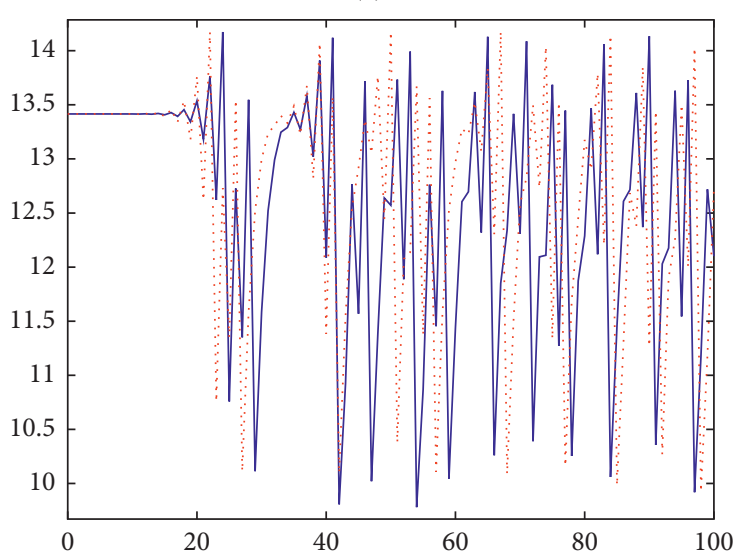

(c)

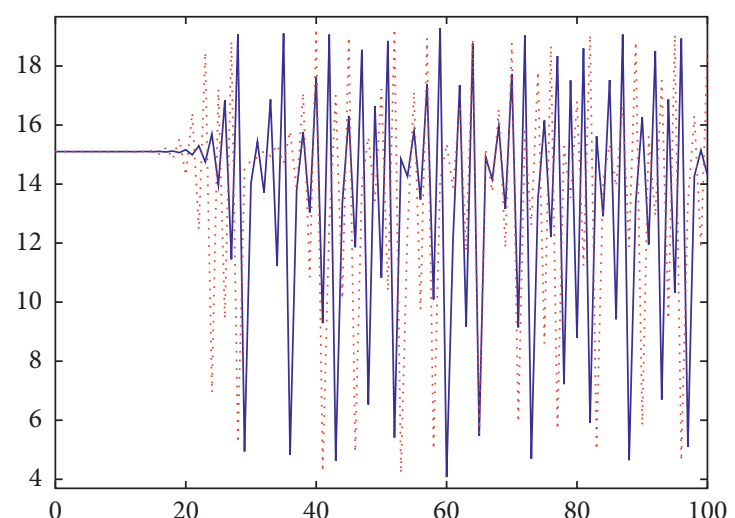

(b)

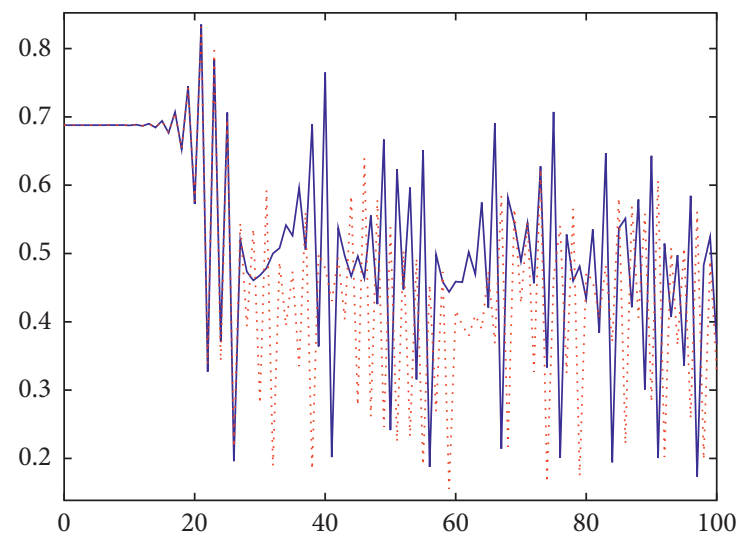

(d)

Figure 8: Chaos attractor and the sensitivity to initial value.

independent retailer or directly to consumers through a direct channel. The retailers sell a store-brand (SB) product when they sell the NB product coming from the manufacturer. So, there is competition both in different channels and in products with different brands. We consider the two Stackelberg game: either the manufacturer acts as a leader or the retailer acts as a leader. We use dynamic theory to analyze the complexity of the model, such as bifurcation and chaos. The results indicate that in the MS model, with the increase of the adjustment speed, both the wholesale price and the direct selling price are higher which in turn incur larger profits for the manufacturer, but it gets worse when the adjustment speed increases. In the RS model, with the increase of the adjustment speed, the increase of the adjustment speed is unfavorable to the retailer. By controlling the total cost of the direct channel and increasing channel competition strength and brand competitive strength, the manufacturers can increase their profits in the game dominated by the retailer. In addition, the stable region within the system will become smaller when the market is sensitive to the channel competition, brand competition, and advertising indifference. The research will serve as a good guidance for the supply chain managers who wish to achieve the best business results. 


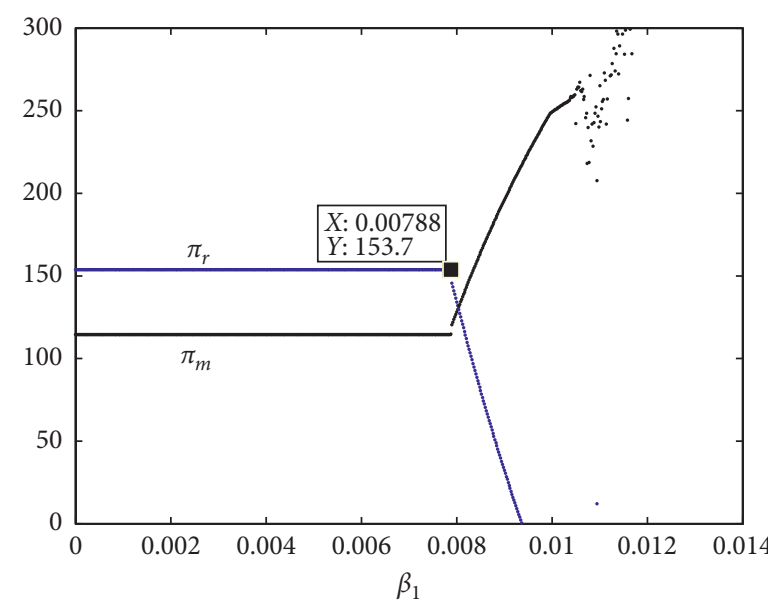

(a)

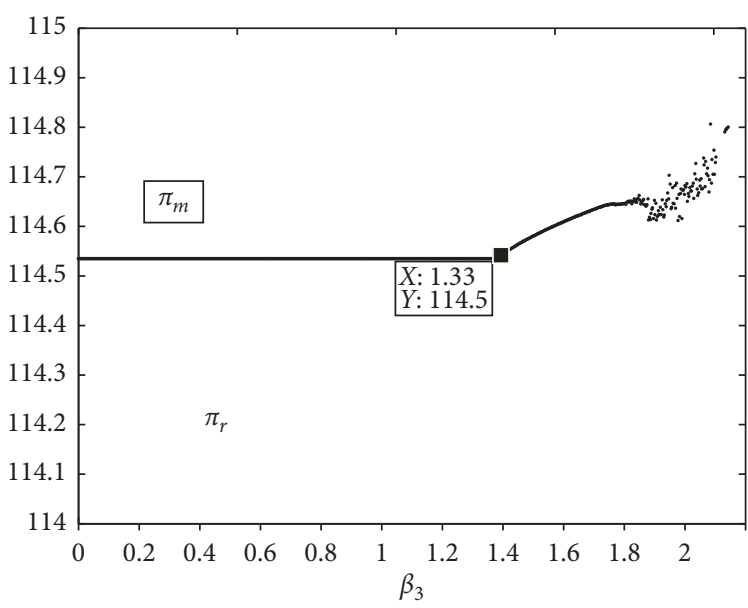

(c)

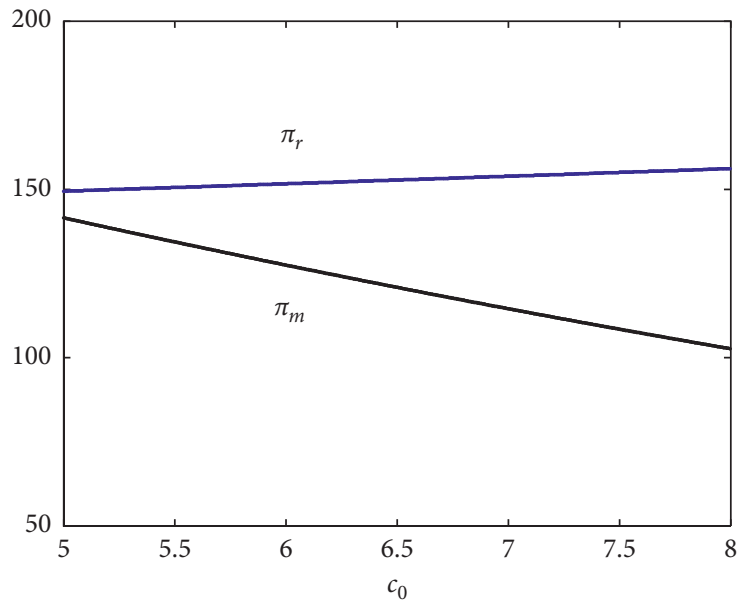

(e)

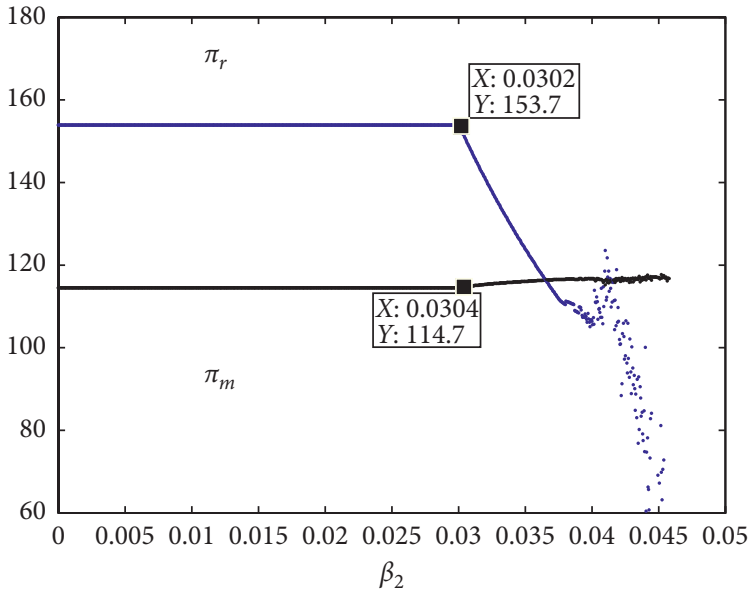

(b)

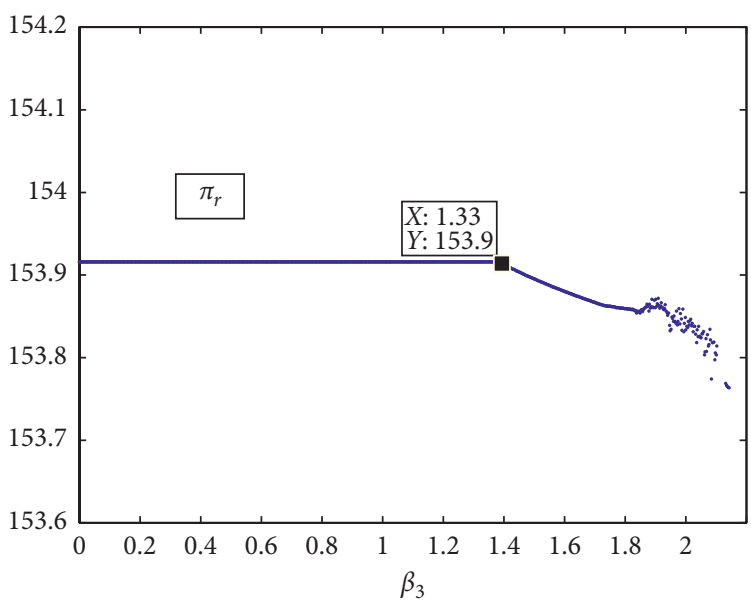

(d)

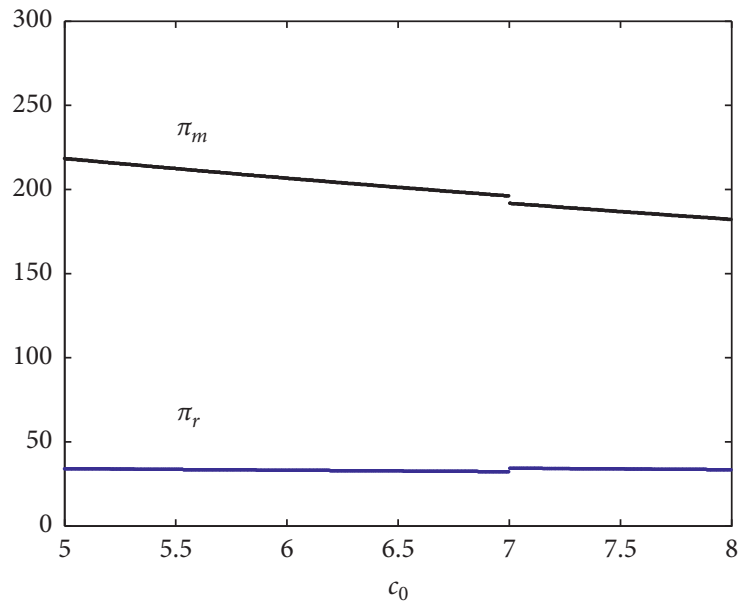

(f)

FIgUre 9: Continued. 


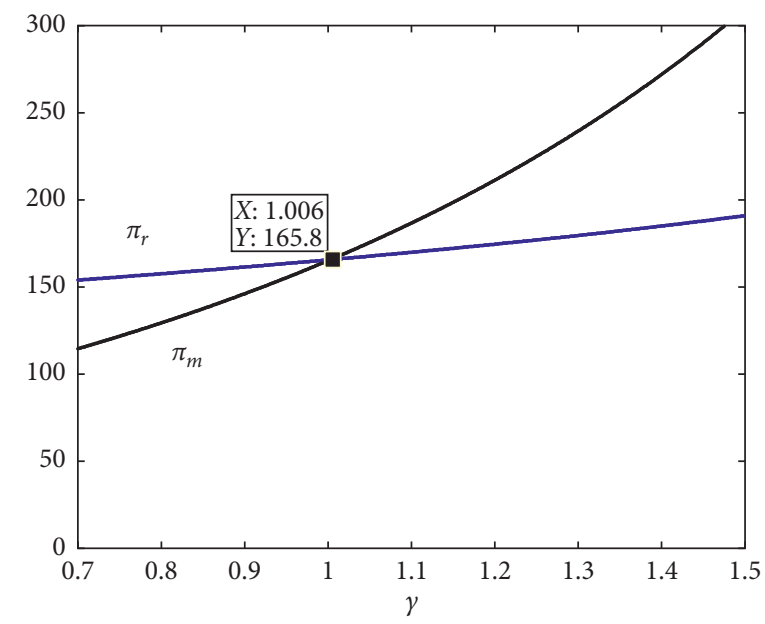

(g)

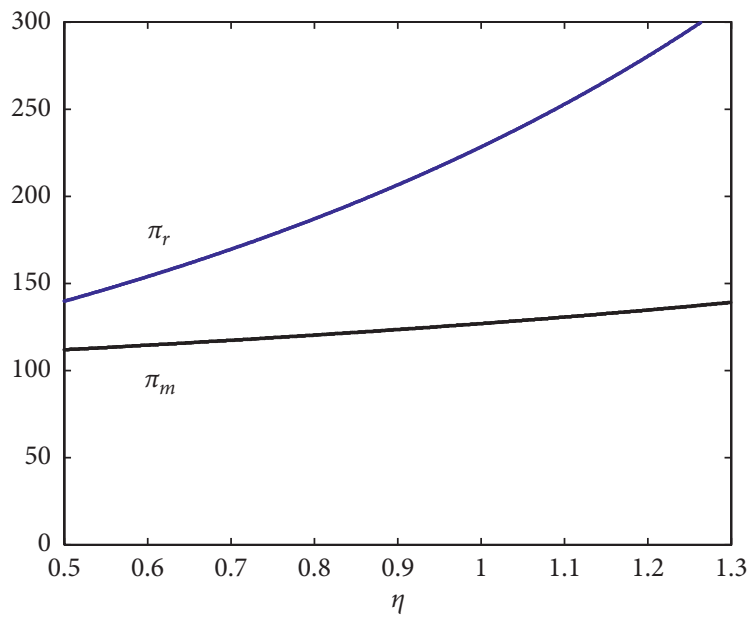

(i)

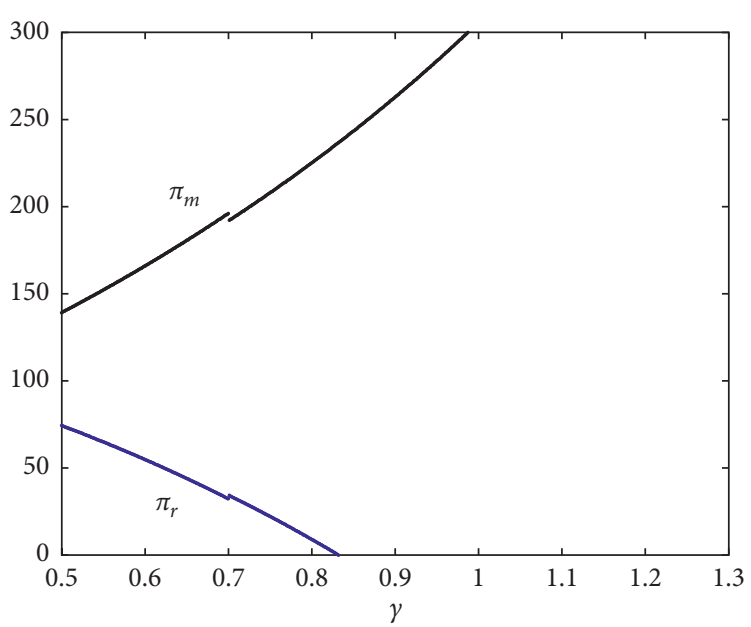

(h)

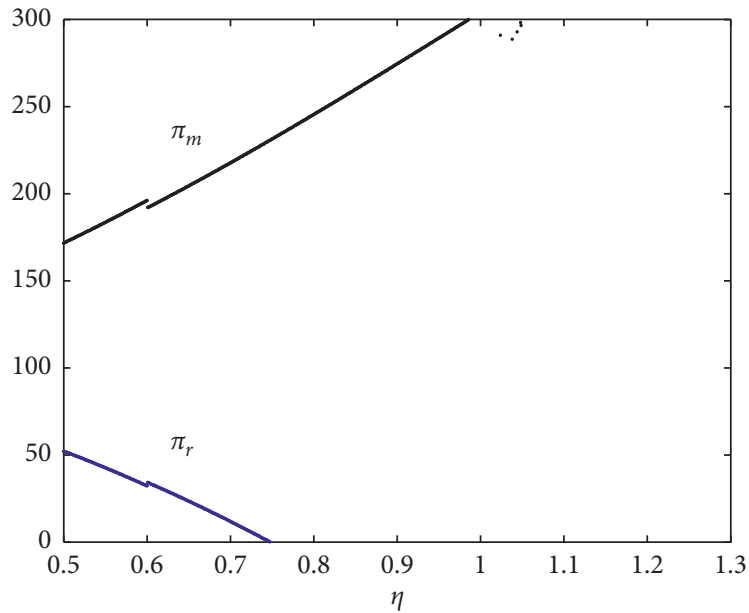

(j)

FIGURE 9: The influence of parameters on the profits.

TABLe 1: Units for magnetic properties.

\begin{tabular}{lcccc}
\hline & MS & MS & RS & RS \\
\hline & $\alpha_{1}=0.01$, & $\alpha_{1}=0.05$, & $\beta_{1}=0.001$, & $\beta_{1}=0.009$, \\
& $\alpha_{2}=0.01$ & $\alpha_{2}=0.01$ & $\beta_{2}=0.01$, & $\beta_{2}=0.01$, \\
$\beta_{3}=0.1$ \\
$p_{0}$ & 11.406 & 10.4333 & 8.1 & 9.7763 \\
$p_{1}$ & 13.3228 & 13.1668 & 13.3215 & 13.3111 \\
$p_{2}$ & 15.0705 & 14.5760 & 15.0997 & 14.0365 \\
$B$ & 13.4116 & 13.3964 & 13.4153 & 12.7192 \\
$\pi_{m}$ & 0.6868 & 0.5634 & 0.6879 & 0.3506 \\
$\pi_{r}$ & 141.4914 & 120.1906 & 112.289 & 192.2792 \\
$\pi$ & 122.3231 & 143.2053 & 150.898 & 31.6133 \\
& 263.8145 & 263.3959 & 263.187 & 223.8925 \\
\hline
\end{tabular}




\section{Appendix}

$$
\begin{aligned}
w^{M}= & \left(2 b _ { 0 } ( 4 b _ { 1 } b _ { 2 } - 4 \eta ^ { 2 } - b _ { 1 } \theta ^ { 2 } - b _ { 2 } \theta ^ { 2 } + 2 \eta \theta ^ { 2 } ) \left(\left(\left(b_{2} c_{0} \gamma+\eta\left(a_{2}-c_{0} \gamma-c_{1} \eta\right) \theta^{2}+b_{1}^{2} c_{1}\left(4 b_{2}-\theta^{2}\right)+c_{2} \eta\left(-4 \eta^{2}-b_{2} \theta^{2}+\eta \theta^{2}\right)\right.\right.\right.\right. \\
& \left.+b_{1}\left(-\left(a_{2}+c_{2} \eta\right) \theta^{2}+2 c_{1} \eta\left(-2 \eta+\theta^{2}\right)+b_{2} c_{2}\left(4 \eta+\theta^{2}\right)\right)\right)+\gamma\left(2 a_{0}\left(4 \eta^{2}+b_{2} \theta^{2}-2 \eta \theta^{2}+b_{1}\left(-4 b_{2}+\theta^{2}\right)\right)^{2}\right. \\
& -\gamma\left(-16 b_{2} c_{2} \eta^{3}+16 c_{1} \eta^{4}+4 b_{2}^{2} c_{0} \gamma \theta^{2}-4 b_{2}^{2} c_{2} \eta \theta^{2}-4 b_{2} c_{0} \gamma \eta \theta^{2}+8 b_{2} c_{2} \eta^{2} \theta^{2}-12 c_{1} \eta^{3} \theta^{2}+b_{2}^{2} c_{2} \theta^{4}-b_{2} c_{0} \gamma \theta^{4}-b_{2} c_{1} \eta \theta^{4}\right. \\
& -2 b_{2} c_{2} \eta \theta^{4}+c_{0} \gamma \eta \theta^{4}+3 c_{1} \eta^{2} \theta^{4}+c_{2} \eta^{2} \theta^{4}+2 b_{1}^{2} c_{1}\left(-4 b_{2}+\theta^{2}\right)^{2}-b_{1}\left(4 b_{2}-\theta^{2}\right)\left(4 a_{2} \eta-4 b_{2} c_{2} \eta+12 c_{1} \eta^{2}+b_{2} c_{1} \theta^{2}-5 c_{1} \eta \theta^{2}\right) \\
& \left.\left.\left.+a_{2}\left(16 \eta^{3}+8 b_{2} \eta \theta^{2}-12 \eta^{2} \theta^{2}-b_{2} \theta^{4}+\eta \theta^{4}\right)\right)\right)\right)+a_{1}\left(-\gamma^{2}\left(b_{2}-\eta\right) \theta^{2}\left(4 b_{2}-\theta^{2}\right)+2 b_{0}\left(b_{1}^{2}\left(-4 b_{2}+\theta^{2}\right)^{2}\right.\right. \\
& \left.\left.\left.\left.+\eta\left(4 \eta-\theta^{2}\right)\left(4 \eta^{2}+b_{2} \theta^{2}-2 \eta \theta^{2}\right)+b_{1}\left(-4 b_{2}^{2} \theta^{2}+\eta \theta^{2}\left(8 \eta-3 \theta^{2}\right)+b_{2}\left(-32 \eta^{2}+12 \eta \theta^{2}+\theta^{4}\right)\right)\right)\right)\right)\right) \\
& \cdot\left(2 \left(2 b _ { 0 } \left(b_{1}^{3}\left(-4 b_{2}+\theta^{2}\right)^{2}+\eta^{2} \theta^{2}\left(4 \eta^{2}+b_{2} \theta^{2}-2 \eta \theta^{2}\right)+b_{1} \eta\left(16 \eta^{3}-16 \eta^{2} \theta^{2}-2 b_{2} \theta^{4}+5 \eta \theta^{4}\right)+b_{1}^{2}\left(-4 b_{2}^{2} \theta^{2}+4 \eta \theta^{2}\left(2 \eta-\theta^{2}\right)\right.\right.\right.\right. \\
& \left.\left.\left.+b_{2}\left(-32 \eta^{2}+16 \eta \theta^{2}+\theta^{4}\right)\right)\right)\right)-\gamma^{2}\left(16 \eta^{4}-16 \eta^{3} \theta^{2}+b_{2}^{2} \theta^{4}-4 b_{2} \eta \theta^{4}+2 b_{1}^{2}\left(-4 b_{2}+\theta^{2}\right)^{2}+\eta^{2}\left(4 b_{2} \theta^{2}+5 \theta^{4}\right)\right. \\
& \left.\left.+b_{1}\left(-8 b_{2}^{2} \theta^{2}+6 \eta \theta^{2}\left(2 \eta-\theta^{2}\right)+b_{2}\left(-48 \eta^{2}+24 \eta \theta^{2}+2 \theta^{4}\right)\right)\right)\right)^{-1}
\end{aligned}
$$

$$
\begin{aligned}
p_{0}^{M}= & \left(2 a _ { 0 } \left(b_{1}^{3}\left(-4 b_{2}+\theta^{2}\right)^{2}+\eta^{2} \theta^{2}\left(4 \eta^{2}+b_{2} \theta^{2}-2 \eta \theta^{2}\right)+b_{1} \eta\left(16 \eta^{3}-16 \eta^{2} \theta^{2}-2 b_{2} \theta^{4}+5 \eta \theta^{4}\right)+b_{1}^{2}\left(-4 b_{2}^{2} \theta^{2}+4 \eta \theta^{2}\left(2 \eta-\theta^{2}\right)\right.\right.\right. \\
& \left.\left.+b_{2}\left(-32 \eta^{2}+16 \eta \theta^{2}+\theta^{4}\right)\right)\right)+2 b_{0} c_{0}\left(b_{1}^{3}\left(-4 b_{2}+\theta^{2}\right)^{2}+\eta^{2} \theta^{2}\left(4 \eta^{2}+b_{2} \theta^{2}-2 \eta \theta^{2}\right)+b_{1} \eta\left(16 \eta^{3}-16 \eta^{2} \theta^{2}-2 b_{2} \theta^{4}+5 \eta \theta^{4}\right)\right. \\
& \left.+b_{1}^{2}\left(-4 b_{2}^{2} \theta^{2}+4 \eta \theta^{2}\left(2 \eta-\theta^{2}\right)+b_{2}\left(-32 \eta^{2}+16 \eta \theta^{2}+\theta^{4}\right)\right)\right)+\gamma\left(-16 c_{0} \gamma \eta^{4}+16 c_{2} \eta^{5}-8 b_{2} c_{0} \gamma \eta^{2} \theta^{2}-8 a_{2} \eta^{3} \theta^{2}+8 b_{2} c_{2} \eta^{3} \theta^{2}\right. \\
& \left.+20 c_{0} \gamma \eta^{3} \theta^{2}-12 c_{2} \eta^{4} \theta^{2}-2 b_{2}^{2} c_{0} \gamma \theta^{4}-\theta^{2}\right)\left(\left(2 c_{0} \gamma+c_{1} \eta-2 c_{2} \eta\right) \theta^{2}+a_{2}\left(4 \eta-2 \theta^{2}\right)+b_{2}\left(-8 c_{0} \gamma+4 c_{2} \eta-c_{1} \theta^{2}+2 c_{2} \theta^{2}\right)\right) \\
& +a_{1}\left(2 b_{1}^{2}\left(-4 b_{2}+\theta^{2}\right)^{2}+\eta\left(16 \eta^{3}-12 \eta^{2} \theta^{2}-b_{2} \theta^{4}+3 \eta \theta^{4}\right)+b_{1}\left(-4 b_{2}^{2} \theta^{2}+\eta \theta^{2}\left(12 \eta-5 \theta^{2}\right)+b_{2}\left(-48 \eta^{2}+20 \eta \theta^{2}+\theta^{4}\right)\right)\right) \\
& +b_{1}\left(-b_{2}^{2} \theta^{2}\left(-12 c_{0} \gamma+c_{2}\left(8 \eta+\theta^{2}\right)\right)+b_{2}\left(\theta^{2}\left(4 a_{2} \eta+4 c_{1} \eta^{2}+a_{2} \theta^{2}-2 c_{1} \eta \theta^{2}\right)+c_{0} \gamma\left(48 \eta^{2}-28 \eta \theta^{2}-3 \theta^{4}\right)\right.\right. \\
& \left.\left.\left.+c_{2}\left(-32 \eta^{3}+4 \eta^{2} \theta^{2}+6 \eta \theta^{4}\right)\right)+\eta\left(a_{2}\left(-16 \eta^{2}+16 \eta \theta^{2}-5 \theta^{4}\right)+\theta^{2}\left(c_{0} \gamma\left(-12 \eta+7 \theta^{2}\right)+\eta\left(-4 c_{1} \eta+12 c_{2} \eta+2 c_{1} \theta^{2}-5 c_{2} \theta^{2}\right)\right)\right)\right)\right) \\
& +\left(2 \left(2 b 0 \left(b_{1}^{3}\left(-4 b_{2}+\theta^{2}\right)^{2}+\eta^{2} \theta^{2}\left(4 \eta^{2}+b_{2} \theta^{2}-2 \eta \theta^{2}\right)+b_{1} \eta\left(16 \eta^{3}-16 \eta^{2} \theta^{2}-2 b_{2} \theta^{4}+5 \eta \theta^{4}\right)\right.\right.\right. \\
& \left.+b_{1}^{2}\left(-4 b_{2}^{2} \theta^{2}+4 \eta \theta^{2}\left(2 \eta-\theta^{2}\right)+b_{2}\left(-32 \eta^{2}+16 \eta \theta^{2}+\theta^{4}\right)\right)\right)-\gamma^{2}\left(16 \eta^{4}-16 \eta^{3} \theta^{2}+b_{2}^{2} \theta^{4}-4 b_{2} \eta \theta^{4}+2 b_{1}^{2}\left(-4 b_{2}+\theta^{2}\right)^{2}\right. \\
& \left.\left.\left.+\eta^{2}\left(4 b_{2} \theta^{2}+5 \theta^{4}\right)+b_{1}\left(-8 b_{2}^{2} \theta^{2}+6 \eta \theta^{2}\left(2 \eta-\theta^{2}\right)+b_{2}\left(-48 \eta^{2}+24 \eta \theta^{2}+2 \theta^{4}\right)\right)\right)\right)\right)^{-1},
\end{aligned}
$$




$$
\begin{aligned}
& p_{1}^{M}=\left(a _ { 1 } \left(-\gamma^{2}\left(4 b_{2}-\theta^{2}\right)\left(b_{1}\left(4 b_{2}-\theta^{2}\right)+\eta\left(-2 \eta+\theta^{2}\right)\right)+b_{0}\left(3 b_{1}^{2}\left(-4 b_{2}+\theta^{2}\right)^{2}+b_{1}\left(-64 b_{2} \eta^{2}-8 b_{2}^{2} \theta^{2}+32 b_{2} \eta \theta^{2}+16 \eta^{2} \theta^{2}\right.\right.\right.\right. \\
& \left.\left.\left.+2 b_{2} \theta^{4}-8 \eta \theta^{4}\right)+\eta\left(16 \eta^{3}-16 \eta^{2} \theta^{2}-2 b_{2} \theta^{4}+5 \eta \theta^{4}\right)\right)\right)+\gamma\left(a _ { 0 } \left(16 \eta^{4}-20 \eta^{3} \theta^{2}+2 b_{2}^{2} \theta^{4}-7 b_{2} \eta \theta^{4}+2 b_{1}^{2}\left(-4 b_{2}+\theta^{2}\right)^{2}\right.\right. \\
& \left.+\eta^{2}\left(8 b_{2} \theta^{2}+7 \theta^{4}\right)+b_{1}\left(-12 b_{2}^{2} \theta^{2}+\eta \theta^{2}\left(12 \eta-7 \theta^{2}\right)+b_{2}\left(-48 \eta^{2}+28 \eta \theta^{2}+3 \theta^{4}\right)\right)\right)-\gamma\left(b_{1}^{2} c_{1}\left(-4 b_{2}+\theta^{2}\right)^{2}\right. \\
& -q b_{1}\left(4 b_{2}-\theta^{2}\right)\left(6 c_{1} \eta^{2}+c_{0} \gamma \theta^{2}-3 c_{1} \eta \theta^{2}+c_{2} \eta \theta^{2}+a_{2}\left(-4 \eta+\theta^{2}\right)+b_{2}\left(-4 c_{0} \gamma+\left(c_{1}-c_{2}\right) \theta^{2}\right)\right) \\
& +\eta\left(-2 b_{2}^{2} c_{2} \theta^{2}-a_{2}\left(8 \eta^{2}-4 \eta \theta^{2}+\theta^{4}\right)+\left(2 \eta-\theta^{2}\right)\left(\left(c_{0} \gamma+c_{2} \eta\right) \theta^{2}+2 c_{1} \eta\left(2 \eta-\theta^{2}\right)\right)+b_{2}\left(4 c_{0} \gamma\left(-2 \eta+\theta^{2}\right)\right.\right. \\
& \left.\left.\left.\left.+\theta^{2}\left(2 a_{2}+2 c_{1} \eta-c_{1} \theta^{2}+c_{2} \theta^{2}\right)\right)\right)\right)\right)+b_{0}\left(b_{1}^{3} c_{1}\left(-4 b_{2}+\theta^{2}\right)^{2}+b_{1}^{2}\left(4 b_{2}-\theta^{2}\right)\left(8 a_{2} \eta-8 c_{1} \eta^{2}-3 a_{2} \theta^{2}-c_{0} \gamma \theta^{2}\right.\right. \\
& \left.+5 c_{1} \eta \theta^{2}-3 c_{2} \eta \theta^{2}+b_{2}\left(4 c_{0} \gamma+4 c_{2} \eta-2 c_{1} \theta^{2}+3 c_{2} \theta^{2}\right)\right)+c_{2} \eta\left(16 \eta^{4}-16 \eta^{3} \theta^{2}+2 b_{2}^{2} \theta^{4}-7 b_{2} \eta \theta^{4}+\eta^{2}\left(12 b_{2} \theta^{2}+5 \theta^{4}\right)\right) \\
& +\theta^{2}\left(-2 b_{2}^{2} c_{0} \gamma \theta^{2}+\eta^{2}\left(-12 a_{2} \eta+4 c_{0} \gamma \eta+4 c_{1} \eta^{2}+5 a_{2} \theta^{2}-2 c_{0} \gamma \theta^{2}-3 c_{1} \eta \theta^{2}\right)+b_{2} \eta\left(-2\left(a_{2}-c_{1} \eta\right) \theta^{2}+c_{0} \gamma\left(-8 \eta+5 \theta^{2}\right)\right)\right) \\
& +b_{1}-2 b_{2}^{2} \theta^{2}\left(-2 c_{0} \gamma+c_{2}\left(6 \eta+\theta^{2}\right)\right)+\eta\left(-4 a_{2}\left(8 \eta^{2}-7 \eta \theta^{2}+2 \theta^{4}\right)+c_{1} \eta\left(16 \eta^{2}-20 \eta \theta^{2}+7 \theta^{4}\right)+\theta^{2}\left(8 c_{2} \eta\left(2 \eta-\theta^{2}\right)\right.\right. \\
& \left.\left.\left.\left.+c_{0} \gamma\left(4 \eta-\theta^{2}\right)\right)\right)+b_{2}\left(-c_{0} \gamma\left(16 \eta^{2}-4 \eta \theta^{2}+\theta^{4}\right)+2\left(c_{2} \eta\left(-16 \eta^{2}+2 \eta \theta^{2}+5 \theta^{4}\right)+\theta^{2}\left(2 c_{1} \eta\left(\eta-\theta^{2}\right)+a_{2}\left(2 \eta+\theta^{2}\right)\right)\right)\right)\right)\right) \\
& \cdot\left(2 \left(2 b _ { 0 } \left(b_{1}^{3}\left(-4 b_{2}+\theta^{2}\right)^{2}+\eta^{2} \theta^{2}\left(4 \eta^{2}+b_{2} \theta^{2}-2 \eta \theta^{2}\right)+b_{1} \eta\left(16 \eta^{3}-16 \eta^{2} \theta^{2}-2 b_{2} \theta^{4}+5 \eta \theta^{4}\right)\right.\right.\right. \\
& \left.+b_{1}^{2}\left(-4 b_{2}^{2} \theta^{2}+4 \eta \theta^{2}\left(2 \eta-\theta^{2}\right)+b_{2}\left(-32 \eta^{2}+16 \eta \theta^{2}+\theta^{4}\right)\right)\right)-\gamma^{2}\left(16 \eta^{4}-16 \eta^{3} \theta^{2}+b_{2}^{2} \theta^{4}-4 b_{2} \eta \theta^{4}+2 b_{1}^{2}\left(-4 b_{2}+\theta^{2}\right)^{2}\right. \\
& \left.\left.\left.+\eta^{2}\left(4 b_{2} \theta^{2}+5 \theta^{4}\right)+b_{1}\left(-8 b_{2}^{2} \theta^{2}+6 \eta \theta^{2}\left(2 \eta-\theta^{2}\right)+b_{2}\left(-48 \eta^{2}+24 \eta \theta^{2}+2 \theta^{4}\right)\right)\right)\right)\right)^{-1},
\end{aligned}
$$

$$
\begin{aligned}
& p_{2}^{M}=\left(a _ { 2 } \left(-\gamma^{2}\left(2 \eta^{2} \theta^{2}+b_{2} \theta^{4}-2 \eta \theta^{4}+8 b_{1}^{2}\left(4 b_{2}-\theta^{2}\right)+b_{1}\left(-24 \eta^{2}-8 b_{2} \theta^{2}+12 \eta \theta^{2}+\theta^{4}\right)\right)+b_{0}\left(-2 b_{1} \eta \theta^{4}+\eta^{2} \theta^{4}+8 b_{1}^{3}\left(4 b_{2}-\theta^{2}\right)\right.\right.\right. \\
& \left.\left.+b_{1}^{2}\left(-32 \eta^{2}-8 b_{2} \theta^{2}+16 \eta \theta^{2}+\theta^{4}\right)\right)\right)-\gamma\left(-8 a_{1} \gamma \eta^{3}-8 c_{0} \gamma^{2} \eta^{3}+24 c_{2} \gamma \eta^{4}-2 a_{1} b_{2} \gamma \eta \theta^{2}-2 b_{2} c_{0} \gamma^{2} \eta \theta^{2}+8 a_{1} \gamma \eta^{2} \theta^{2}\right. \\
& +4 b_{2} c_{2} \gamma \eta^{2} \theta^{2}+8 c_{0} \gamma^{2} \eta^{2} \theta^{2}+2 c_{1} \gamma \eta^{3} \theta^{2}-24 c_{2} \gamma \eta^{3} \theta^{2}+a_{1} b_{2} \gamma \theta^{4}+b_{2}^{2} c_{2} \gamma \theta^{4}+b_{2} c_{0} \gamma^{2} \theta^{4}-a_{0} b_{2} \eta \theta^{4}-2 a_{1} \gamma \eta \theta^{4}-5 b_{2} c_{2} \gamma \eta \theta^{4} \\
& -2 c_{0} \gamma^{2} \eta \theta^{4}+a_{0} \eta^{2} \theta^{4}-c_{1} \gamma \eta^{2} \theta^{4}+8 c_{2} \gamma \eta^{2} \theta^{4}+b_{1}^{2}\left(4 b_{2}-\theta^{2}\right)\left(8 b_{2} c_{2} \gamma-4 a_{0} \eta+\left(c_{1}-4 c_{2}\right) \gamma \theta^{2}\right) \\
& +b_{1}\left(16 a_{0} \eta^{3}-8 b_{2}^{2} c_{2} \gamma \theta^{2}-4 c_{0} \gamma^{2} \eta \theta^{2}-8 a_{0} \eta^{2} \theta^{2}-4 c_{1} \gamma \eta^{2} \theta^{2}+20 c_{2} \gamma \eta^{2} \theta^{2}+c_{0} \gamma^{2} \theta^{4}-a_{0} \eta \theta^{4}+2 c_{1} \gamma \eta \theta^{4}-11 c_{2} \gamma \eta \theta^{4}\right. \\
& \left.\left.-a_{1} \gamma\left(4 b_{2}-\theta^{2}\right)\left(-4 \eta+\theta^{2}\right)+b_{2}\left(4 c_{0} \gamma^{2}\left(4 \eta-\theta^{2}\right)+\theta^{2}\left(4 a_{0} \eta-2 c_{1} \gamma \eta+a_{0} \theta^{2}\right)+c_{2} \gamma\left(-56 \eta^{2}+32 \eta \theta^{2}+3 \theta^{4}\right)\right)\right)\right) \\
& +b_{0}\left(b_{1}^{3}\left(4 b_{2}-\theta^{2}\right)\left(8 b_{2} c_{2}+\left(c_{1}-4 c_{2}\right) \theta^{2}\right)+\eta \theta^{2}\left(a_{1} \eta\left(-4 \eta+\theta^{2}\right)-c_{0} \gamma\left(4 \eta^{2}+b_{2} \theta^{2}-2 \eta \theta^{2}\right)\right.\right. \\
& \left.+\eta\left(12 c_{2} \eta^{2}+3 b_{2} c_{2} \theta^{2}+c_{1} \eta \theta^{2}-7 c_{2} \eta \theta^{2}\right)\right)+b_{1}^{2}\left(-8 b_{2}^{2} c_{2} \theta^{2}-a_{1}\left(4 b_{2}-\theta^{2}\right)\left(-8 \eta+\theta^{2}\right)\right. \\
& \left.+b_{2}\left(-64 c_{2} \eta^{2}-4 c_{1} \eta \theta^{2}+44 c_{2} \eta \theta^{2}+3 c_{2} \theta^{4}+4 c_{0} \gamma\left(4 \eta-\theta^{2}\right)\right)+\theta^{2}\left(c_{0} \gamma\left(-4 \eta+\theta^{2}\right)+\eta\left(-4 c_{1} \eta+24 c_{2} \eta+3 c_{1} \theta^{2}-15 c_{2} \theta^{2}\right)\right)\right) \\
& -b_{1}\left(2 a_{1} \eta\left(16 \eta^{2}+2 b_{2} \theta^{2}-10 \eta \theta^{2}+\theta^{4}\right)+c_{0} \gamma\left(16 \eta^{3}-12 \eta^{2} \theta^{2}-b_{2} \theta^{4}+3 \eta \theta^{4}\right)+\eta\left(c_{1} \eta \theta^{2}\left(-4 \eta+3 \theta^{2}\right)\right.\right. \\
& \left.\left.\left.\left.+c_{2}\left(-32 \eta^{3}+4 b_{2} \eta \theta^{2}+44 \eta^{2} \theta^{2}+6 b_{2} \theta^{4}-18 \eta \theta^{4}\right)\right)\right)\right)\right) \cdot\left(2 \left(2 b _ { 0 } \left(b_{1}^{3}\left(-4 b_{2}+\theta^{2}\right)^{2}+\eta^{2} \theta^{2}\left(4 \eta^{2}+b_{2} \theta^{2}-2 \eta \theta^{2}\right)\right.\right.\right. \\
& \left.+b_{1} \eta\left(16 \eta^{3}-16 \eta^{2} \theta^{2}-2 b_{2} \theta^{4}+5 \eta \theta^{4}\right)+b_{1}^{2}\left(-4 b_{2}^{2} \theta^{2}+4 \eta \theta^{2}\left(2 \eta-\theta^{2}\right)+b_{2}\left(-32 \eta^{2}+16 \eta \theta^{2}+\theta^{4}\right)\right)\right) \\
& -\gamma^{2}\left(16 \eta^{4}-16 \eta^{3} \theta^{2}+b_{2}^{2} \theta^{4}-4 b_{2} \eta \theta^{4}+2 b_{1}^{2}\left(-4 b_{2}+\theta^{2}\right)^{2}+\eta^{2}\left(4 b_{2} \theta^{2}+5 \theta^{4}\right)+b_{1}\left(-8 b_{2}^{2} \theta^{2}+6 \eta \theta^{2}\left(2 \eta-\theta^{2}\right)\right.\right. \\
& \left.\left.\left.\left.+b_{2}\left(-48 \eta^{2}+24 \eta \theta^{2}+2 \theta^{4}\right)\right)\right)\right)\right)^{-1} \text {, }
\end{aligned}
$$




$$
\begin{aligned}
& B^{M}=\left(\theta \left(8 b_{0} b_{1}^{3} b_{2}^{2} c_{1}-16 b_{0} b_{1}^{3} b_{2}^{2} c_{2}-8 b_{0} b_{1}^{2} b_{2}^{2} c_{0} \gamma-8 b_{1}^{2} b_{2}^{2} c_{1} \gamma^{2}+16 b_{1}^{2} b_{2}^{2} c_{2} \gamma^{2}+8 b_{1} b_{2}^{2} c_{0} \gamma^{3}+8 b_{0} b_{1}^{2} b_{2}^{2} c_{2} \eta+8 a_{0} b_{1}^{2} b_{2} \gamma \eta\right.\right. \\
& +8 b_{0} b_{1}^{2} b_{2} c_{0} \gamma \eta-8 b_{1} b_{2}^{2} c_{2} \gamma^{2} \eta-8 b_{1} b_{2} c_{0} \gamma^{3} \eta-16 b_{0} b_{1}^{2} b_{2} c_{1} \eta^{2}+32 b_{0} b_{1}^{2} b_{2} c_{2} \eta^{2}-8 a_{0} b_{1} b_{2} \gamma \eta^{2}+8 b_{0} b_{1} b_{2} c_{0} \gamma \eta^{2} \\
& +12 b_{1} b_{2} c_{1} \gamma^{2} \eta^{2}-20 b_{1} b_{2} c_{2} \gamma^{2} \eta^{2}-4 b_{2} c_{0} \gamma^{3} \eta^{2}-16 b_{0} b_{1} b_{2} c_{2} \eta^{3}-8 a_{0} b_{1} \gamma \eta^{3}-8 b_{0} b_{1} c_{0} \gamma \eta^{3}+8 b_{2} c_{2} \gamma^{2} \eta^{3}+4 c_{0} \gamma^{3} \eta^{3} \\
& +8 b_{0} b_{1} c_{1} \eta^{4}-16 b_{0} b_{1} c_{2} \eta^{4}+8 a_{0} \gamma \eta^{4}-4 c_{1} \gamma^{2} \eta^{4}+4 c_{2} \gamma^{2} \eta^{4}+8 b_{0} c_{2} \eta^{5}-2 b_{0} b_{1}^{3} b_{2} c_{1} \theta^{2}+4 b_{0} b_{1}^{3} b_{2} c_{2} \theta^{2}+2 b_{0} b_{1}^{2} b_{2}^{2} c_{2} \theta^{2} \\
& -2 a_{0} b_{1} b_{2}^{2} \gamma \theta^{2}+2 b_{0} b_{1}^{2} b_{2} c_{0} \gamma \theta^{2}+2 b_{0} b_{1} b_{2}^{2} c_{0} \gamma \theta^{2}+2 b_{1}^{2} b_{2} c_{1} \gamma^{2} \theta^{2}-4 b_{1}^{2} b_{2} c_{2} \gamma^{2} \theta^{2}-2 b_{1} b_{2}^{2} c_{2} \gamma^{2} \theta^{2}-2 b_{1} b_{2} c_{0} \gamma^{3} \theta^{2}-2 b_{2}^{2} c_{0} \gamma^{3} \theta^{2} \\
& +4 b_{0} b_{1}^{2} b_{2} c_{1} \eta \theta^{2}-10 b_{0} b_{1}^{2} b_{2} c_{2} \eta \theta^{2}-2 b_{0} b_{1} b_{2}^{2} c_{2} \eta \theta^{2}-2 a_{0} b_{1}^{2} \gamma \eta \theta^{2}-2 b_{0} b_{1}^{2} c_{0} \gamma \eta \theta^{2}-6 b_{0} b_{1} b_{2} c_{0} \gamma \eta \theta^{2}-3 b_{1} b_{2} c_{1} \gamma^{2} \eta \theta^{2} \\
& +8 b_{1} b_{2} c_{2} \gamma^{2} \eta \theta^{2}+b_{2}^{2} c_{2} \gamma^{2} \eta \theta^{2}+2 b_{1} c_{0} \gamma^{3} \eta \theta^{2}+5 b_{2} c_{0} \gamma^{3} \eta \theta^{2}+2 b_{0} b_{1}^{2} c_{1} \eta^{2} \theta^{2}-2 b_{0} b_{1} b_{2} c_{1} \eta^{2} \theta^{2}-4 b_{0} b_{1}^{2} c_{2} \eta^{2} \theta^{2}+4 b_{0} b_{1} b_{2} c_{2} \eta^{2} \theta^{2} \\
& +6 a_{0} b_{1} \gamma \eta^{2} \theta^{2}+4 a_{0} b_{2} \gamma \eta^{2} \theta^{2}+4 b_{0} b_{1} c_{0} \gamma \eta^{2} \theta^{2}-b_{1} c_{1} \gamma^{2} \eta^{2} \theta^{2}+b_{2} c_{1} \gamma^{2} \eta^{2} \theta^{2}+2 b_{1} c_{2} \gamma^{2} \eta^{2} \theta^{2}-2 b_{2} c_{2} \gamma^{2} \eta^{2} \theta^{2}-3 c_{0} \gamma^{3} \eta^{2} \theta^{2} \\
& -4 b_{0} b_{1} c_{1} \eta^{3} \theta^{2}+10 b_{0} b_{1} c_{2} \eta^{3} \theta^{2}+2 b_{0} b_{2} c_{2} \eta^{3} \theta^{2}-6 a_{0} \gamma \eta^{3} \theta^{2}+c_{1} \gamma^{2} \eta^{3} \theta^{2}-3 c_{2} \gamma^{2} \eta^{3} \theta^{2}+2 b_{0} c_{1} \eta^{4} \theta^{2}-6 b_{0} c_{2} \eta^{4} \theta^{2} \\
& +a_{1}\left(\gamma^{2}\left(b_{2}-\eta\right)\left(8 b_{1} b_{2}-4 \eta^{2}-2 b_{1} \theta^{2}-2 b_{2} \theta^{2}+3 \eta \theta^{2}\right)-2 b_{0}\left(b_{1}^{2}\left(b_{2}-2 \eta\right)\left(4 b_{2}-\theta^{2}\right)+b_{1} \eta\left(8 \eta^{2}+3 b_{2} \theta^{2}-5 \eta \theta^{2}\right)\right.\right. \\
& \left.\left.+\eta^{2}\left(-4 \eta^{2}-2 b_{2} \theta^{2}+3 \eta \theta^{2}\right)\right)\right)+a_{2}\left(-2 b_{0}\left(b_{1}-\eta\right)\left(\eta^{2} \theta^{2}+b_{1}^{2}\left(-8 b_{2}+2 \theta^{2}\right)+b_{1}\left(8 \eta^{2}+b_{2} \theta^{2}-4 \eta \theta^{2}\right)\right)+\gamma^{2}\left(4 b_{1}^{2}\left(-4 b_{2}+\theta^{2}\right)\right.\right. \\
& \left.\left.\left.\left.+2 b_{1}\left(8 b_{2} \eta+6 \eta^{2}+b_{2} \theta^{2}-5 \eta \theta^{2}\right)+\eta\left(-12 \eta^{2}-3 b_{2} \theta^{2}+7 \eta \theta^{2}\right)\right)\right)\right)\right) \cdot\left(2 \left(2 b _ { 0 } \left(b_{1}^{3}\left(-4 b_{2}+\theta^{2}\right)^{2}+\eta^{2} \theta^{2}\left(4 \eta^{2}+b_{2} \theta^{2}-2 \eta \theta^{2}\right)\right.\right.\right. \\
& +b_{1} \eta\left(16 \eta^{3}-16 \eta^{2} \theta^{2}-2 b_{2} \theta^{4}+5 \eta \theta^{4}+b_{1}^{2}\left(-4 b_{2}^{2} \theta^{2}+4 \eta \theta^{2}\left(2 \eta-\theta 2^{2}\right)+b_{2}\left(-32 \eta^{2}+16 \eta \theta^{2}+\theta^{4}\right)\right)\right) \gamma^{2}\left(16 \eta^{4}-16 \eta^{3} \theta^{2}\right. \\
& \left.\left.+b_{2}^{2} \theta^{4}-4 b_{2} \eta \theta^{4}+2 b_{1}^{2}\left(-4 b_{2}+\theta^{2}\right)^{2}+\eta^{2}\left(4 b_{2} \theta^{2}+5 \theta^{4}\right)+b_{1}\left(-8 b_{2}^{2} \theta^{2}+6 \eta \theta^{2}\left(2 \eta-\theta^{2}\right)+b_{2}\left(-48 \eta^{2}+24 \eta \theta^{2} 2 \theta^{4}\right)\right)\right)\right)^{-1} \text {. }
\end{aligned}
$$

Proof of Proposition 1. We substitute $E_{1}$ into (19) and then get two eigenvalues:

$$
\begin{aligned}
r_{1}= & \left(1+\left(\alpha _ { 1 } \left(4 c_{0} \gamma \eta^{2}-4 c_{2} \eta^{3}+2 b_{2} c_{0} \gamma \theta^{2}+a_{2} \eta \theta^{2}-b_{2} c_{2} \eta \theta^{2}-3 c_{0} \gamma \eta \theta^{2}-c_{1} \eta^{2} \theta^{2}+c_{2} \eta^{2} \theta^{2}+b_{1}^{2} c_{1}\left(4 b_{2}-\theta^{2}\right)+\alpha_{1}\left(b_{1}\left(4 b_{2}-\theta^{2}\right)\right.\right.\right.\right. \\
& \left.\left.\left.\left.+\eta\left(-4 \eta+\theta^{2}\right)\right)+b_{1}\left(-a_{2} \theta^{2}+c_{0} \gamma \theta^{2}-c_{2} \eta \theta^{2}+2 c_{1} \eta\left(-2 \eta+\theta^{2}\right)+b_{2}\left(-4 c_{0} \gamma+4 c_{2} \eta+c_{2} \theta^{2}\right)\right)\right)\right)\right) \\
& \cdot\left(8 b_{1} b_{2}-8 \eta^{2}-2 b_{1} \theta^{2}-2 b_{2} \theta^{2}+4 \eta \theta^{2}\right)^{-1}, \\
r_{2}= & \left(1+\left(\alpha _ { 2 } \left(2 b_{0} c_{0}\left(4 b_{1} b_{2}-4 \eta^{2}-b_{1} \theta^{2}-b_{2} \theta^{2}+2 \eta \theta^{2}\right)+a_{0}\left(8 b_{1} b_{2}-8 \eta^{2}-2 b_{1} \theta^{2}-2 b_{2} \theta^{2}+4 \eta \theta^{2}\right)\right.\right.\right. \\
& \left.\left.\left.-\gamma\left(4 b_{2} c_{0} \gamma-4 a_{2} \eta-4 c_{1} \eta^{2}+a_{2} \theta^{2}-b_{2} c_{2} \theta^{2}-c_{0} \gamma \theta^{2}+c 1 \eta \theta^{2}+c_{2} \eta \theta^{2}+b_{1} c_{1}\left(4 b_{2}-\theta^{2}\right)+\alpha_{1}\left(-4 b_{2}+\theta^{2}\right)\right)\right)\right)\right) \\
& \cdot\left(8 b_{1} b_{2}-8 \eta^{2}-2 b_{1} \theta^{2}-2 b_{2} \theta^{2}+4 \eta \theta^{2}\right)^{-1} .
\end{aligned}
$$

Comparing the eigenvalue with the wholesale price $w$ in the equilibrium point $E_{2}$ and the direct sale price $p_{0}$ in the equilibrium point $E_{3}$ and noting the condition (13), we draw the conclusion that $\left|r_{1,2}\right|>1$. So, $E_{1}$ is unstable. 
For $E_{2}$, we calculate the eigenvalue of its Jacobian matrix to obtain

$$
\begin{aligned}
& r_{1}=\left(1-\left(\alpha _ { 1 } \left(4 c_{0} \gamma \eta^{2}-4 c_{2} \eta^{3}+2 b_{2} c_{0} \gamma \theta^{2}+a_{2} \eta \theta^{2}-b_{2} c_{2} \eta \theta^{2}-3 c_{0} \gamma \eta \theta^{2}-c_{1} \theta^{2}+c_{2} \eta^{2} \theta^{2}+b_{1}^{2} c_{1}\left(4 b_{2}-\theta^{2}\right)+\alpha_{1}\left(b_{1}\left(4 b_{2}-\theta^{2}\right)\right.\right.\right.\right. \\
& \left.\left.\left.\left.+\eta\left(-4 \eta+\theta^{2}\right)\right)+b_{1}\left(-\left(a_{2}-c_{0} \gamma+c_{2} \eta\right) \theta^{2}+2 c_{1} \eta\left(-2 \eta+\theta^{2}\right)+b_{2}\left(-4 c_{0} \gamma+c_{2}\left(4 \eta+\theta^{2}\right)\right)\right)\right)\right)\right) \\
& \cdot\left(8 b_{1} b_{2}-8 \eta^{2}-2 b_{1} \theta^{2}-2 b_{2} \theta^{2}+4 \eta \theta^{2}\right)^{-1} \text {, } \\
& r_{2}=\left(1+\left(\alpha _ { 2 } \left(2 a _ { 0 } \left(b_{1}^{3}\left(-4 b_{2}+\theta^{2}\right)^{2}+\eta^{2} \theta^{2}\left(4 \eta^{2}+b_{2} \theta^{2}-2 \eta \theta^{2}\right)+b_{1} \eta\left(16 \eta^{3}-16 \eta^{2} \theta^{2}-2 b_{2} \theta^{4}+5 \eta \theta^{4}\right)\right.\right.\right.\right. \\
& \left.+b_{1}^{2}\left(-4 b_{2}^{2} \theta^{2}+4 \eta \theta^{2}\left(2 \eta-\theta^{2}\right)+b_{2}\left(-32 \eta^{2}+16 \eta \theta^{2}+\theta^{4}\right)\right)\right)+2 b_{0} c_{0}\left(b_{1}^{3}\left(-4 b_{2}+\theta^{2}\right)^{2}+\eta^{2} \theta^{2}\left(4 \eta^{2}+b_{2} \theta^{2}-2 \eta \theta^{2}\right)\right. \\
& \left.+b_{1} \eta\left(16 \eta^{3}-16 \eta^{2} \theta^{2}-2 b_{2} \theta^{4}+5 \eta \theta^{4}\right)+b_{1}^{2}\left(-4 b_{2}^{2} \theta^{2}+4 \eta \theta^{2}\left(2 \eta-\theta^{2}\right)+b_{2}\left(-32 \eta^{2}+16 \eta \theta^{2}+\theta^{4}\right)\right)\right) \\
& +\gamma\left(16 c_{0} \gamma \eta^{4}+16 c_{2} \eta^{5}-8 b_{2} c_{0} \gamma \eta^{2} \theta^{2}-8 a_{2} \eta^{3} \theta^{2}+8 b_{2} c_{2} \eta^{3} \theta^{2}+20 c_{0} \gamma \eta^{3} \theta^{2}-12 c_{2} \eta^{4} \theta^{2}-2 b_{2}^{2} c_{0} \gamma \theta^{4}-a_{2} b_{2} \eta \theta^{4}+b_{2}^{2} c_{2} \eta \theta^{4}\right. \\
& +7 b_{2} c_{0} \gamma \eta \theta^{4}+3 a_{2} \eta^{2} \theta^{4}+b_{2} c_{1} \eta^{2} \theta^{4}-4 b_{2} c_{2} \eta^{2} \theta^{4}-7 c_{0} \gamma \eta^{2} \theta^{4}-c_{1} \eta^{3} \theta^{4}+3 c_{2} \eta^{3} \theta^{4}+b_{1}^{2}\left(4 b_{2}-\theta^{2}\right) \\
& \cdot\left(\left(2 c_{0} \gamma+c_{1} \eta-2 c_{2} \eta\right) \theta^{2}+a_{2}\left(4 \eta-2 \theta^{2}\right)+b_{2}\left(-8 c_{0} \gamma+4 c_{2} \eta-c_{1} \theta^{2}+2 c_{2} \theta^{2}\right)\right)+a_{1}\left(2 b_{1}^{2}\left(-4 b_{2}+\theta^{2}\right)^{2}\right. \\
& \left.+\eta\left(16 \eta^{3}-12 \eta^{2} \theta^{2}-b_{2} \theta^{4}+3 \eta \theta^{4}\right)+b_{1}\left(-4 b_{2}^{2} \theta^{2}+\eta \theta^{2}\left(12 \eta-5 \theta^{2}\right)+b_{2}\left(-48 \eta^{2}+20 \eta \theta^{2}+\theta^{4}\right)\right)\right) \\
& +b_{1}\left(-b_{2}^{2} \theta^{2}\left(-12 c_{0} \gamma+c_{2}\left(8 \eta+\theta^{2}\right)\right)+b_{2}\left(\theta^{2}\left(4 a_{2} \eta+4 c_{1} \eta^{2}+a_{2} \theta^{2}-2 c_{1} \eta \theta^{2}\right)+c_{0} \gamma\left(48 \eta^{2}-28 \eta \theta^{2}-3 \theta^{4}\right)\right.\right. \\
& \left.+c_{2}\left(-32 \eta^{3}+4 \eta^{2} \theta^{2}+6 \eta \theta^{4}\right)\right)+\eta\left(a_{2}\left(-16 \eta^{2}+16 \eta \theta^{2}-5 \theta^{4}\right)+\theta^{2}\left(c_{0} \gamma\left(-12 \eta+7 \theta^{2}\right)\right.\right. \\
& \left.\left.\left.\left.\left.\left.\left.+\eta\left(-4 c_{1} \eta+12 c_{2} \eta+2 c_{1} \theta^{2}-5 c_{2} \theta^{2}\right)\right)\right)\right)\right)\right)\right)\right) \cdot\left(2\left(4 b_{1} b_{2}-4 \eta^{2}-b_{1} \theta^{2}-b_{2} \theta^{2}+2 \eta \theta^{2}\right)\left(-\eta^{2} \theta^{2}+b_{1}^{2}\left(4 b_{2}-\theta^{2}\right)+2 b_{1} \eta\left(-2 \eta+\theta^{2}\right)\right)\right)^{-1} \text {. }
\end{aligned}
$$

According to the assumption, the wholesale price $w$ in the equilibrium point $E_{2}$ is bigger than zero. Comparing $w$ and $r_{1}$, we have $\left|r_{1}\right|<1$. For $r_{2}$, because $p_{0}^{M}>0$ and based on condition (6), we have $\left|r_{2}\right|>1$. So, $E_{2}$ is unstable.

$$
\begin{aligned}
r_{1}= & \left(1+\left(\alpha _ { 1 } \left(2 b _ { 0 } ( 4 b _ { 1 } b _ { 2 } - 4 \eta ^ { 2 } - b _ { 1 } \theta ^ { 2 } - b _ { 2 } \theta ^ { 2 } + 2 \eta \theta ^ { 2 } ) \left(\left(b_{2} c_{0} \gamma+\eta\left(a_{2}-c_{0} \gamma-c_{1} \eta\right)\right) \theta^{2}+b_{1}^{2} c_{1}\left(4 b_{2}-\theta^{2}\right)+c_{2} \eta\left(-4 \eta^{2}-b_{2} \theta^{2}+\eta \theta^{2}\right)\right.\right.\right.\right. \\
& \left.+b_{1}\left(-\left(a_{2}+c_{2} \eta\right) \theta^{2}+2 c_{1} \eta\left(-2 \eta+\theta^{2}\right)+b_{2} c_{2}\left(4 \eta+\theta^{2}\right)\right)\right)+\gamma\left(2 a_{0}\left(4 \eta^{2}+b_{2} \theta^{2}-2 \eta \theta^{2}+b_{1}\left(-4 b_{2}+\theta^{2}\right)\right)^{2}\right. \\
& -\gamma\left(-16 b_{2} c_{2} \eta^{3}+16 c_{1} \eta^{4}+4 b_{2}^{2} c_{0} \gamma \theta^{2}-4 b_{2}^{2} c_{2} \eta \theta^{2}-4 b_{2} c_{0} \gamma \eta \theta^{2}+8 b_{2} c_{2} \eta^{2} \theta^{2}-12 c_{1} \eta^{3} \theta^{2}+b_{2}^{2} c_{2} \theta^{4}-b_{2} c_{0} \gamma \theta^{4}-b_{2} c_{1} \eta \theta^{4}\right. \\
& -2 b_{2} c_{2} \eta \theta^{4}+c_{0} \gamma \eta \theta^{4}+3 c_{1} \eta^{2} \theta^{4}+c_{2} \eta^{2} \theta^{4}+2 b_{1}^{2} c_{1}\left(-4 b_{2}+\theta^{2}\right)^{2}-b_{1}\left(4 b_{2}-\theta^{2}\right)\left(4 a_{2} \eta-4 b_{2} c_{2} \eta+12 c_{1} \eta^{2}+b_{2} c_{1} \theta^{2}-5 c_{1} \eta \theta^{2}\right) \\
& \left.\left.+a_{2}\left(16 \eta^{3}+8 b_{2} \eta \theta^{2}-12 \eta^{2} \theta^{2}-b_{2} \theta^{4}+\eta \theta^{4}\right)\right)\right)+a_{1}\left(-\gamma^{2}\left(b_{2}-\eta\right) \theta^{2}\left(4 b_{2}-\theta^{2}\right)+2 b_{0}\left(b_{1}^{2}\left(-4 b_{2}+\theta^{2}\right)^{2}+\eta\left(4 \eta-\theta^{2}\right)\right.\right. \\
& \left.\left.\left.\left.\left.\cdot\left(4 \eta^{2}+b_{2} \theta^{2}-2 \eta \theta^{2}\right)+b_{1}\left(-4 b_{2}^{2} \theta^{2}+\eta \theta^{2}\left(8 \eta-3 \theta^{2}\right)+b_{2}\left(-32 \eta^{2}+12 \eta \theta^{2}+\theta^{4}\right)\right)\right)\right)\right)\right)\right) \\
& \cdot\left(2\left(4 b_{1} b_{2}-4 \eta^{2}-b_{1} \theta^{2} b_{2} \theta^{2}+2 \eta \theta^{2}\right)\left(\gamma^{2}\left(-4 b_{2}+\theta^{2}\right)+b_{0}\left(8 b_{1} b_{2}-8 \eta^{2}-2 b_{1} \theta^{2}-2 b_{2} \theta^{2}+4 \eta \theta^{2}\right)\right)\right)^{-1}, \\
r_{2}= & \left(1-\left(\alpha _ { 2 } \left(2 b_{0} c_{0}\left(4 b_{1} b_{2}-4 \eta^{2}-b_{1} \theta^{2}-b_{2} \theta^{2}+2 \eta \theta^{2}\right)+a_{0}\left(8 b_{1} b_{2}-8 \eta^{2}-2 b_{1} \theta^{2}-2 b_{2} \theta^{2}+4 \eta \theta^{2}\right)+\gamma\left(4 a_{1} b_{2}-4 b_{1} b_{2} c_{1}-4 b_{2} c_{0} \gamma\right.\right.\right.\right. \\
& \left.\left.\left.\left.+4 a_{2} \eta+4 c_{1} \eta^{2}-a_{1} \theta^{2}-a_{2} \theta^{2}+b_{1} c_{1} \theta^{2}+b_{2} c_{2} \theta^{2}+c_{0} \gamma \theta^{2}-c_{1} \eta \theta^{2}-c_{2} \eta \theta^{2}\right)\right)\right)\right) \cdot\left(8 b_{1} b_{2}-8 \eta^{2}-2 b_{1} \theta^{2}-2 b_{2} \theta^{2}+4 \eta \theta^{2}\right)^{-1} .
\end{aligned}
$$


For $r_{1}$, using condition (10) and $\omega^{M}>0$, we have $\left|r_{1}\right|>1$. We also have $\left|r_{2}\right|<1$ by comparing the direct sale price $p_{0}$ in the equilibrium point $E_{3}$. So, $E_{3}$ is unstable too.
Proof of Proposition 2. We substitute $E_{1}$ into (36) and then get one eigenvalue:

$$
r_{1}=1+\beta_{1}\left(\frac{a_{1}\left(3 b_{0} b_{1}-\gamma^{2}\right)+b_{0} b_{1}\left(b_{1} c_{1}+c_{0} \gamma-c_{2} \eta\right)+\gamma\left(2 a_{0} b_{1}-\gamma\left(b_{1} c_{1}+c_{0} \gamma-c_{2} \eta\right)\right)}{b_{0} b_{1}-\gamma^{2}}\right) .
$$

Comparing $r_{1}$ with $p_{1}$ in the $E_{4}$, we draw the conclusion that $\left|r_{1}\right|>1$. So, $E_{1}$ is unstable.
For $E_{2}$, we calculate one eigenvalue of its Jacobian matrix to obtain

$$
\begin{aligned}
r_{1}= & \left(1+\beta_{1}\left(a_{1}\left(2 b_{2} \gamma^{4}+2 b_{0}^{2} b_{1}\left(3 b_{1} b_{2}-\eta^{2}\right)+b_{0} \gamma^{2}\left(-8 b_{1} b_{2}+\eta^{2}\right)\right)+\gamma^{3}\left(a_{0}\left(-4 b_{1} b_{2}+\eta^{2}\right)+\gamma\left(2 b_{1} b_{2} c_{1}+2 b_{2} c_{0} \gamma+2 a_{2} \eta-c_{1} \eta^{2}\right)\right)\right.\right. \\
& +2 b_{0}^{2} b_{1}\left(b_{1}^{2} b_{2} c_{1}-c_{2} \eta^{3}+b_{1}\left(b_{2} c_{0} \gamma+2 a_{2} \eta+b_{2} c_{2} \eta-c_{1} \eta^{2}\right)\right)+b_{0} \gamma\left(2 a_{0} b_{1}\left(2 b_{1} b_{2}-\eta^{2}\right)+\gamma\left(-4 b_{1}^{2} b_{2} c_{1}+c_{2} \eta^{3}\right.\right. \\
& \left.\left.\left.\left.-b_{1}\left(4 b_{2} c_{0} \gamma+6 a_{2} \eta+2 b_{2} c_{2} \eta-3 c_{1} \eta^{2}\right)\right)\right)\right)\right) \cdot\left(2 b_{2} b_{0} b_{1}-\gamma^{2}+b_{0} \eta^{2}\left(2 b_{0} b_{1}-\gamma^{2}\right)\right)^{-1} .
\end{aligned}
$$

Comparing $r_{1}$ with $p_{1}$ in the $E_{6}$, we have that $\left|r_{1}\right|>1$. So, $E_{2}$ is unstable.
For $E_{3}$, one eigenvalue is

$$
\begin{aligned}
r_{1}= & \left(1+\beta_{1}\left(4 a_{1}\left(3 b_{0}^{2} b_{1}^{2}-4 b_{0} b_{1} \gamma^{2}+\gamma^{4}\right)+\gamma^{3}\left(a_{0}\left(-8 b_{1}+\theta^{2}\right)+\gamma\left(4 b_{1} c_{1}+4 c_{0} \gamma-4 c_{2} \eta-c_{1} \theta^{2}+2 c_{2} \theta^{2}\right)\right)\right.\right. \\
& +b_{0}^{2} b_{1}\left(4 b_{1}^{2} c_{1}+\left(c_{0} \gamma-4 c_{2} \eta\right) \theta^{2}+b_{1}\left(4 c_{0} \gamma-4 c_{2} \eta-2 c_{1} \theta^{2}+6 c_{2} \theta^{2}\right)\right)-b_{0} \gamma\left(a_{0} b 1\left(-8 b 1+\theta^{2}\right)+\gamma\left(8 b_{1}^{2} c_{1}+\left(c_{0} \gamma-2 c_{2} \eta\right) \theta^{2}\right.\right. \\
& \left.\left.\left.\left.+b_{1}\left(8 c_{0} \gamma-8 c_{2} \eta-3 c_{1} \theta^{2}+8 c_{2} \theta^{2}\right)\right)\right)\right)\right) \cdot\left(4 \gamma^{4}+4 b_{0}^{2} b_{1}\left(b_{1}+\theta^{2}\right)-2 b_{0} \gamma^{2}\left(4 b_{1}+\theta^{2}\right)\right)^{-1}
\end{aligned}
$$

Comparing $r_{1}$ with $p_{1}$ in the $E_{7}$, we have that $\left|r_{1}\right|>1$. So, $E_{3}$ is unstable.
For $E_{4}$, we get one eigenvalue as follows:

$$
r_{2}=\frac{1+\beta_{2}\left(2 a_{2} b_{1}\left(b_{0} b_{1}-\gamma^{2}\right)+b_{0} b_{1}\left(2 b_{1} b_{2} c_{2}+\eta\left(2 a_{1}+c_{0} \gamma-2 c_{2} \eta\right)\right)+\gamma\left(b_{1}\left(-2 b_{2} c_{2} \gamma+a_{0} \eta\right)-\gamma \eta\left(a_{1}+c_{0} \gamma-c_{2} \eta\right)\right)\right)}{\left(2 b_{1}\left(b_{0} b_{1}-\gamma^{2}\right)\right)} .
$$

Comparing $r_{2}$ with $p_{2}$ in the $E_{6}$, we have that $\left|r_{2}\right|>1$. So,

For $E_{5}$, one eigenvalue of its Jacobian matrix is $E_{4}$ is unstable. 


$$
\begin{aligned}
r_{1}= & \left(1+\beta_{1}\left(\gamma ^ { 3 } \left(\gamma \left(8 b_{1} b_{2} c_{1}+8 b_{2} c_{0} \gamma+8 a_{2} \eta-4 c_{1} \eta^{2}-2 a_{2} \theta^{2}-2 b_{1} c_{1} \theta^{2}-2 b_{2} c_{1} \theta^{2}+2 b_{2} c_{2} \theta^{2}+a_{1}\left(2 \gamma^{4}\left(4 b_{2}-\theta^{2}\right)\right.\right.\right.\right.\right. \\
& \left.-2 b_{0} \gamma^{2}\left(4 b_{1}\left(4 b_{2}-\theta^{2}\right)+\eta\left(-2 \eta+\theta^{2}\right)\right)+2 b_{0}^{2} b_{1}\left(3 b_{1}\left(4 b_{2}-\theta^{2}\right)+2 \eta\left(-2 \eta+\theta^{2}\right)\right)\right)+b_{0}^{2} b_{1}\left(\left(2 b_{2} c_{0} \gamma+4 a_{2} \eta-c_{0} \gamma \eta\right) \theta^{2}\right. \\
& +b_{1}^{2}\left(8 b_{2} c_{1}-2 c_{1} \theta^{2}\right)-4 c_{2} \eta\left(2 \eta^{2}+b_{2} \theta^{2}-\eta \theta^{2}\right)+2 b_{1}\left(8 a_{2} \eta-4 c_{1} \eta^{2}-3 a_{2} \theta^{2}-c_{0} \gamma \theta^{2}+3 c_{1} \eta \theta^{2}-3 c_{2} \eta \theta^{2}\right. \\
& \left.\left.+b_{2}\left(4 c_{0} \gamma+4 c_{2} \eta-2 c_{1} \theta^{2}+3 c_{2} \theta^{2}\right)\right)\right)+b_{0} \gamma\left(a_{0} b_{1}\left(16 b_{1} b_{2}-8 \eta^{2}-4 b_{1} \theta^{2}-2 b_{2} \theta^{2}+5 \eta \theta^{2}\right)+\gamma\left(\left(-2 b_{2} c_{0} \gamma-2 a_{2} \eta+c_{0} \gamma \eta\right) \theta^{2}\right.\right. \\
& +4 b_{1}^{2} c_{1}\left(-4 b_{2}+\theta^{2}\right)+2 c_{2} \eta\left(2 \eta^{2}+b_{2} \theta^{2}-\eta \theta^{2}\right)+b_{1}\left(-24 a_{2} \eta+12 c_{1} \eta^{2}+8 a_{2} \theta^{2}+4 c_{0} \gamma \theta^{2}-9 c_{1} \eta \theta^{2}+8 c_{2} \eta \theta^{2}\right. \\
& \left.\left.\left.\left.\left.\left.\left.-2 b_{2}\left(8 c_{0} \gamma-3 c_{1} \theta^{2}+4 c_{2}\left(\eta+\theta^{2}\right)\right)\right)\right)\right)\right)\right)\right)\right) \cdot\left(2 \gamma^{4}\left(4 b_{2}-\theta^{2}\right)+2 b_{0}^{2} b_{1}\left(4 b_{1} b_{2}+4 \eta^{2}-b_{1} \theta^{2}+4 b_{2} \theta^{2}-4 \eta \theta^{2}\right)\right. \\
& \left.-4 b_{0} \gamma^{2}\left(4 b_{1} b_{2}+\eta^{2}-b_{1} \theta^{2}+b_{2} \theta^{2}-\eta \theta^{2}\right)\right)^{-1} .
\end{aligned}
$$

Comparing $r_{1}$ with $p_{1}$ in the $E^{R}$, we have that $\left|r_{1}\right|>1$. So, $E_{5}$ is unstable.
For $E_{6}$, we get one eigenvalue as follows:

$$
\begin{aligned}
r_{3}= & \left(1+\beta_{3}\left(\left(2 b_{0}^{2} b_{1}^{3} b_{2} c_{1}-4 b_{0}^{2} b_{1}^{3} b_{2} c_{2}-2 b_{0}^{2} b_{1}^{2} b_{2} c_{0} \gamma-4 b_{0} b_{1}^{2} b_{2} c_{1} \gamma^{2}+8 b_{0} b_{1}^{2} b_{2} c_{2} \gamma^{2}+4 b_{0} b_{1} b_{2} c_{0} \gamma^{3}+2 b_{1} b_{2} c_{1} \gamma^{4}-4 b_{1} b_{2} c_{2} \gamma^{4}\right.\right.\right. \\
& -2 b_{2} c_{0} \gamma^{5}+2 b_{0}^{2} b_{1}^{2} b_{2} c_{2} \eta+2 a_{0} b_{0} b_{1}^{2} \gamma \eta+2 b_{0}^{2} b_{1}^{2} c_{0} \gamma \eta-2 b_{0} b_{1} b_{2} c_{2} \gamma^{2} \eta-2 a_{0} b_{1} \gamma^{3} \eta-4 b_{0} b_{1} c_{0} \gamma^{3} \eta+2 c_{0} \gamma^{5} \eta-2 b_{0}^{2} b_{1}^{2} c_{1} \eta^{2} \\
& +4 b_{0}^{2} b_{1}^{2} c_{2} \eta^{2}-2 a_{0} b_{0} b_{1} \gamma \eta^{2}+3 b_{0} b_{1} c_{1} \gamma^{2} \eta^{2}-6 b_{0} b_{1} c_{2} \gamma^{2} \eta^{2}+a_{0} \gamma^{3} \eta^{2}-c_{1} \gamma^{4} \eta^{2}+2 c_{2} \gamma^{4} \eta^{2}-2 b_{0}^{2} b_{1} c_{2} \eta^{3}+b_{0} c_{2} \gamma^{2} \eta^{3} \\
& +2 a_{2}\left(b_{0} b_{1}-\gamma^{2}\right)\left(2 b_{0} b_{1}\left(b_{1}-\eta\right)+\gamma^{2}\left(-2 b_{1}+\eta\right)\right)+a_{1}\left(2 \gamma^{4}\left(-b_{2}+\eta\right)+b_{0} \gamma^{2}\left(4 b_{1} b_{2}-6 b_{1} \eta+\eta^{2}\right)\right. \\
& \left.\left.\left.\left.-2 b_{0}^{2} b_{1}\left(b_{1} b_{2}-2 b_{1} \eta+\eta^{2}\right)\right)\right) \theta\right)\right) \cdot\left(4\left(b_{0} b_{1}-\gamma^{2}\right)\left(2 b_{0} b_{1}\left(b_{1} b_{2}-\eta^{2}\right)+\gamma^{2}\left(-2 b_{1} b_{2}+\eta^{2}\right)\right)\right)^{-1} .
\end{aligned}
$$

For $E_{7}$, there is one eigenvalue of its Jacobian matrix, as

Comparing $r_{3}$ with $B$ in the $E^{R}$, we have that $\left|r_{3}\right|>1$. So, $E_{6}$ is unstable.

$$
\begin{aligned}
r_{2}= & \left(1+\beta_{2}\left(2 a_{2}\left(-b_{0} b_{1}+\gamma^{2}\right)^{2}\left(8 b_{1}-\theta^{2}\right)+\gamma^{3}\left(8 a_{1} \gamma \eta+8 c_{0} \gamma^{2} \eta-8 c_{2} \gamma \eta^{2}-2 a_{1} \gamma \theta^{2}-2 b_{2} c_{2} \gamma \theta^{2}-2 c_{0} \gamma^{2} \theta^{2}+a_{0} \eta \theta^{2}-c_{1} \gamma \eta \theta^{2}\right.\right.\right. \\
& \left.+6 c_{2} \gamma \eta \theta^{2}+2 b_{1}\left(8 b_{2} c_{2} \gamma-4 a_{0} \eta+\left(c_{1}-4 c_{2}\right) \gamma \theta^{2}\right)\right)+b_{0}^{2} b_{1}\left(\eta\left(c_{0} \gamma-4 c_{2} \eta\right) \theta^{2}+2 b_{1}^{2}\left(8 b_{2} c_{2}+\left(c_{1}-4 c_{2}\right) \theta^{2}\right)\right. \\
& \left.+2 b_{1}\left(8 a_{1} \eta+4 c_{0} \gamma \eta-8 c_{2} \eta^{2}-a_{1} \theta^{2}-b_{2} c_{2} \theta^{2}-c_{0} \gamma \theta^{2}-c_{1} \eta \theta^{2}+7 c_{2} \eta \theta^{2}\right)\right)+b_{0} \gamma\left(\gamma \eta\left(-c_{0} \gamma+2 c_{2} \eta\right) \theta^{2}-4 b_{1}^{2}\left(8 b_{2} c_{2} \gamma-2 a_{0} \eta\right.\right. \\
& \left.\left.\left.\left.+\left(c_{1}-4 c_{2}\right) \gamma \theta^{2}\right)+b_{1}\left(24 c_{2} \gamma \eta^{2}+4 b_{2} c_{2} \gamma \theta^{2}-a_{0} \eta \theta^{2}+3 c_{1} \gamma \eta \theta^{2}-20 c_{2} \gamma \eta \theta^{2}+4 a_{1} \gamma\left(-6 \eta+\theta^{2}\right)+4 c_{0} \gamma^{2}\left(-4 \eta+\theta^{2}\right)\right)\right)\right)\right) \\
& +\left(2\left(\left(-b_{0} b_{1}+\gamma^{2}\right)^{2}\left(8 b_{1}-\theta^{2}\right)\right)\right)^{-1} .
\end{aligned}
$$

Comparing $r_{2}$ with $p_{2}$ in the $E^{R}$, we have that $\left|r_{2}\right|>1$. So,

$E_{7}$ is unstable: 


$$
\begin{aligned}
p_{1}^{R}= & \left(\gamma ^ { 3 } \left(\gamma\left(8 b_{1} b_{2} c_{1}+8 b_{2} c_{0} \gamma+8 a_{2} \eta-4 c_{1} \eta^{2}-2 a_{2} \theta^{2}-2 b_{1} c_{1} \theta^{2}-2 b_{2} c_{1} \theta^{2}+2 b_{2} c_{2} \theta^{2}-2 c_{0} \gamma \theta^{2}+3 c_{1} \eta \theta^{2}-2 c_{2} \eta \theta^{2}\right)\right.\right. \\
& \left.+a_{0}\left(4 \eta^{2}+2 b_{2} \theta^{2}-3 \eta \theta^{2}+4 b_{1}\left(-4 b_{2}+\theta^{2}\right)\right)\right)+a_{1}\left(2 \gamma^{4}\left(4 b_{2}-\theta^{2}\right)-2 b_{0} \gamma^{2}\left(4 b_{1}\left(4 b_{2}-\theta^{2}\right)+\eta\left(-2 \eta+\theta^{2}\right)\right)\right. \\
& \left.+2 b_{0}^{2} b_{1}\left(3 b_{1}\left(4 b_{2}-\theta^{2}\right)+2 \eta\left(-2 \eta+\theta^{2}\right)\right)\right)+b_{0}^{2} b_{1}\left(\left(2 b_{2} c_{0} \gamma+4 a_{2} \eta-c_{0} \gamma \eta\right) \theta^{2}+b_{1}^{2}\left(8 b_{2} c_{1}-2 c_{1} \theta^{2}\right)-4 c_{2} \eta\left(2 \eta^{2}+b_{2} \theta^{2}-\eta \theta^{2}\right)\right. \\
& \left.+2 b_{1}\left(8 a_{2} \eta-4 c_{1} \eta^{2}-3 a_{2} \theta^{2}-c_{0} \gamma \theta^{2}+3 c_{1} \eta \theta^{2}-3 c_{2} \eta \theta^{2}+b_{2}\left(4 c_{0} \gamma+4 c_{2} \eta-2 c_{1} \theta^{2}+3 c_{2} \theta^{2}\right)\right)\right) \\
& +b_{0} \gamma\left(a_{0} b_{1}\left(16 b_{1} b_{2}-8 \eta^{2}-4 b_{1} \theta^{2}-2 b_{2} \theta^{2}+5 \eta \theta^{2}\right)+\gamma\left(\left(-2 b_{2} c_{0} \gamma-2 a 2 \eta+c_{0} \gamma \eta\right) \theta^{2}+4 b_{1}^{2} c_{1}\left(-4 b_{2}+\theta^{2}\right)+2 c_{2} \eta\left(2 \eta^{2}\right.\right.\right. \\
& \left.\left.\left.\left.+b_{2} \theta^{2}-\eta \theta^{2}\right)+b_{1}\left(-24 a_{2} \eta+12 c_{1} \eta^{2}+8 a_{2} \theta^{2}+4 c_{0} \gamma \theta^{2}-9 c_{1} \eta \theta^{2}+8 c_{2} \eta \theta^{2}-2 b_{2}\left(8 c_{0} \gamma-3 c_{1} \theta^{2}+4 c_{2}\left(\eta+\theta^{2}\right)\right)\right)\right)\right)\right) \\
& +\left(2 \left(2 \gamma^{4}\left(8 b_{1} b_{2}-4 \eta^{2}-2 b_{1} \theta^{2}-b_{2} \theta^{2}+2 \eta \theta^{2}\right)-2 b_{0}^{2} b_{1}\left(\eta^{2} \theta^{2}+b_{1}^{2}\left(-8 b_{2}+2 \theta^{2}\right)+b_{1}\left(8 \eta^{2}+b_{2} \theta^{2}-4 \eta \theta^{2}\right)\right)\right.\right. \\
& \left.\left.+b_{0} \gamma^{2}\left(\eta^{2} \theta^{2}+8 b_{1}^{2}\left(-4 b_{2}+\theta^{2}\right)+4 b_{1}\left(6 \eta^{2}+b_{2} \theta^{2}-3 \eta \theta^{2}\right)\right)\right)\right)^{-1}
\end{aligned}
$$

$$
\begin{aligned}
p_{2}^{R}= & \left(2 a_{2}\left(-b_{0} b_{1}+\gamma^{2}\right)^{2}\left(8 b_{1}-\theta^{2}\right)+\gamma^{3}\left(8 a_{1} \gamma \eta+8 c_{0} \gamma^{2} \eta-8 c_{2} \gamma \eta^{2}-2 a_{1} \gamma \theta^{2}-2 b_{2} c_{2} \gamma \theta^{2}-2 c_{0} \gamma^{2} \theta^{2}+a_{0} \eta \theta^{2}-c_{1} \gamma \eta \theta^{2}\right.\right. \\
& \left.+6 c_{2} \gamma \eta \theta^{2}+2 b_{1}\left(8 b_{2} c_{2} \gamma-4 a_{0} \eta+\left(c_{1}-4 c_{2}\right) \gamma \theta^{2}\right)\right)+b_{0}^{2} b_{1}\left(\eta\left(c_{0} \gamma-4 c_{2} \eta\right) \theta^{2}+2 b_{1}^{2}\left(8 b_{2} c_{2}+\left(c_{1}-4 c_{2}\right) \theta^{2}\right)\right. \\
& \left.+2 b_{1}\left(8 a_{1} \eta+4 c_{0} \gamma \eta-8 c_{2} \eta^{2}-a_{1} \theta^{2}-b_{2} c_{2} \theta^{2}-c_{0} \gamma \theta^{2}-c_{1} \eta \theta^{2}+7 c_{2} \eta \theta^{2}\right)\right)+b_{0} \gamma\left(\gamma \eta\left(-c_{0} \gamma+2 c_{2} \eta\right) \theta^{2}-4 b_{1}^{2}\left(8 b_{2} c_{2} \gamma-2 a_{0} \eta\right.\right. \\
& \left.\left.\left.+\left(c_{1}-4 c_{2}\right) \gamma \theta^{2}\right)+b_{1}\left(24 c_{2} \gamma \eta^{2}+4 b_{2} c_{2} \gamma \theta^{2}-a_{0} \eta \theta^{2}+3 c_{1} \gamma \eta \theta^{2}-20 c_{2} \gamma \eta \theta^{2}+4 a_{1} \gamma\left(-6 \eta+\theta^{2}\right)+4 c_{0} \gamma^{2}\left(-4 \eta+\theta^{2}\right)\right)\right)\right) \\
& +\left(2 \left(2 \gamma^{4}\left(8 b_{1} b_{2}-4 \eta^{2}-2 b_{1} \theta^{2}-b_{2} \theta^{2}+2 \eta \theta^{2}\right)-2 b_{0}^{2} b_{1}\left(\eta^{2} \theta^{2}+b_{1}^{2}\left(-8 b_{2}+2 \theta^{2}\right)+b_{1}\left(8 \eta^{2}+b_{2} \theta^{2}-4 \eta \theta^{2}\right)\right)\right.\right. \\
& \left.\left.+b_{0} \gamma^{2}\left(\eta^{2} \theta^{2}+8 b 1^{2}\left(-4 b_{2}+\theta^{2}\right)+4 b_{1}\left(6 \eta^{2}+b_{2} \theta^{2}-3 \eta \theta^{2}\right)\right)\right)\right)^{-1}
\end{aligned}
$$

$B^{R}=\left(\left(2 b_{0}^{2} b_{1}^{3} b_{2} c 1-4 b_{0}^{2} b_{1}^{3} b_{2} c_{2}-2 b_{0}^{2} b_{1}^{2} b_{2} c_{0} \gamma-4 b_{0} b_{1}^{2} b_{2} c_{1} \gamma^{2}+8 b_{0} b_{1}^{2} b_{2} c_{2} \gamma^{2}+4 b_{0} b_{1} b_{2} c_{0} \gamma^{3}+2 b_{1} b_{2} c_{1} \gamma^{4}-4 b_{1} b_{2} c_{2} \gamma^{4}-2 b_{2} c_{0} \gamma^{5}\right.\right.$ $+2 b_{0}^{2} b_{1}^{2} b_{2} c_{2} \eta+2 a_{0} b_{0} \gamma \eta+2 b_{0}^{2} b_{1}^{2} c_{0} \gamma \eta-2 b_{0} b_{1} b_{2} c_{2} \gamma^{2} \eta-2 a_{0} \gamma^{3} \eta-4 b_{0} b_{1} c_{0} \gamma^{3} \eta+2 c_{0} \gamma^{5} \eta-2 b_{0}^{2} b_{1}^{2} c_{1} \eta^{2}+4 b_{0}^{2} b_{1}^{2} c_{2} \eta^{2}$

$-2 a_{0} b_{0} b_{1} \gamma \eta^{2}+3 b_{0} b_{1} c_{1} \gamma^{2} \eta^{2}-6 b_{0} b_{1} c_{2} \gamma^{2} \eta^{2}+a_{0} \gamma^{3} \eta^{2}-c_{1} \gamma^{4} \eta^{2}+2 c_{2} \gamma^{4} \eta^{2}-2 b_{0}^{2} b_{1} c_{2} \eta^{3}+b_{0} c_{2} \gamma^{2} \eta^{3} 2 a_{2}\left(b_{0} b_{1}-\gamma^{2}\right)$ $\left.\left.\cdot\left(2 b_{0} b_{1}\left(b_{1}-\eta\right)+\gamma^{2}\left(-2 b_{1}+\eta\right)\right)+a_{1}\left(2 \gamma^{4}\left(-b_{2}+\eta\right)+b_{0} \gamma^{2}\left(4 b_{1} b_{2}-6 b_{1} \eta+\eta^{2}\right)-2 b_{0}^{2} b_{1}\left(b_{1} b_{2}-2 b_{1} \eta+\eta^{2}\right)\right)\right) \theta\right)$ $\cdot\left(2 \gamma^{4}\left(8 b_{1} b_{2}-4 \eta^{2}-2 b_{1} \theta^{2}-b_{2} \theta^{2}+2 \eta \theta^{2}\right)-2 b_{0}^{2} b_{1}\left(\eta^{2} \theta^{2}+b_{1}^{2}\left(-8 b_{2}+2 \theta^{2}\right)+b_{1}\left(8 \eta^{2}+b_{2} \theta^{2}-4 \eta \theta^{2}\right)\right)\right.$ $\left.+b_{0} \gamma^{2}\left(\eta^{2} \theta^{2}+8 b_{1}^{2}\left(-4 b_{2}+\theta^{2}\right)+4 b_{1}\left(6 \eta^{2}+b_{2} \theta^{2}-3 \eta \theta^{2}\right)\right)\right)^{-1}$

$$
\begin{aligned}
w^{R}= & \left(2 a_{1}\left(2 b_{0} \gamma^{2} \eta^{2}+\gamma^{4}\left(-4 b_{2}+\theta^{2}\right)+b_{0}^{2} b_{1}\left(b_{1}\left(4 b_{2}-\theta^{2}\right)+\eta\left(-4 \eta+\theta^{2}\right)\right)\right)+\gamma^{3}\left(a_{0}\left(12 \eta^{2}+2 b_{2} \theta^{2}-5 \eta \theta^{2}+4 b_{1}\left(-4 b_{2}+\theta^{2}\right)\right)\right.\right. \\
& \left.+\gamma\left(-8 a_{2} \eta-12 c_{1} \eta^{2}+2 a_{2} \theta^{2}+2 c_{0} \gamma \theta^{2}+5 c_{1} \eta \theta^{2}+2 c_{2} \eta \theta^{2}+6 b_{1} c_{1}\left(4 b_{2}-\theta^{2}\right)-2 b_{2}\left(4 c_{0} \gamma+\left(c_{1}+c_{2}\right) \theta^{2}\right)\right)\right) \\
& +b_{0} \gamma\left(-a_{0}\left(\eta^{2} \theta^{2}+4 b_{1}^{2}\left(-4 b_{2}+\theta^{2}\right)+b_{1}\left(16 \eta^{2}+2 b_{2} \theta^{2}-7 \eta \theta^{2}\right)\right)+\gamma\left(12 b_{1}^{2} c_{1}\left(-4 b_{2}+\theta^{2}\right)+\eta^{2}\left(4 c_{2} \eta+c_{1} \theta^{2}\right)\right.\right. \\
& \left.\left.+c_{0} \gamma\left(-8 \eta^{2}-2 b_{2} \theta^{2}+5 \eta \theta^{2}\right)+b_{1}\left(8 a_{2} \eta+36 c_{1} \eta^{2}-4 c_{0} \gamma \theta^{2}-17 c_{1} \eta \theta^{2}+2 b_{2}\left(8 c_{0} \gamma-4 c_{2} \eta+3 c_{1} \theta^{2}\right)\right)\right)\right) \\
& +b_{0}^{2}\left(c_{0} \gamma \eta^{2} \theta^{2}+6 b_{1}^{3} c_{1}\left(4 b_{2}-\theta^{2}\right)-2 b_{1}^{2}\left(\left(a_{2}-c_{0} \gamma+c_{2} \eta\right) \theta^{2}+6 c_{1} \eta\left(2 \eta-\theta^{2}\right)+b_{2}\left(4 c_{0} \gamma+2 c_{1} \theta^{2}-c_{2}\left(4 \eta+\theta^{2}\right)\right)\right)\right. \\
& \left.\left.+b_{1}\left(c_{0} \gamma\left(8 \eta^{2}+2 b_{2} \theta^{2}-5 \eta \theta^{2}\right)-2 \eta\left(\left(-a_{2}+c_{1} \eta\right) \theta^{2}+c_{2}\left(4 \eta^{2}+b_{2} \theta^{2}-\eta \theta^{2}\right)\right)\right)\right)\right) \\
& +\left(2 \left(2 \gamma^{4}\left(8 b_{1} b_{2}-4 \eta^{2}-2 b_{1} \theta^{2}-b_{2} \theta^{2}+2 \eta \theta^{2}\right)-2 b_{0}^{2} b_{1}\left(\eta^{2} \theta^{2}+b_{1}^{2}\left(-8 b_{2}+2 \theta^{2}\right)+b_{1}\left(8 \eta^{2}+b_{2} \theta^{2}-4 \eta \theta^{2}\right)\right)\right.\right. \\
& \left.\left.+b_{0} \gamma^{2}\left(\eta^{2} \theta^{2}+8 b_{1}^{2}\left(-4 b_{2}+\theta^{2}\right)+4 b_{1}\left(6 \eta^{2}+b_{2} \theta^{2}-3 \eta \theta^{2}\right)\right)\right)\right)^{-1}
\end{aligned}
$$




$$
\begin{aligned}
p_{0}^{R}= & \left(-4 b_{0}^{2} b_{1} c_{0}\left(\eta^{2} \theta^{2}+b_{1}^{2}\left(-8 b_{2}+2 \theta^{2}\right)+b_{1}\left(8 \eta^{2}+b_{2} \theta^{2}-4 \eta \theta^{2}\right)\right)+a_{0}\left(-4 b_{0} b_{1}\left(\eta^{2} \theta^{2}+b_{1}^{2}\left(-8 b_{2}+2 \theta^{2}\right)+b_{1}\left(8 \eta^{2}+b_{2} \theta^{2}-4 \eta \theta^{2}\right)\right)\right.\right. \\
& +\gamma^{2}\left(\eta^{2} \theta^{2}+8 b_{1}^{2}\left(-4 b_{2}+\theta^{2}\right)+4 b_{1}\left(6 \eta^{2}+b_{2} \theta^{2}-b_{1}^{2}\left(-8 b_{2}+2 \theta^{2}\right)+b_{1}\left(8 \eta^{2}+b_{2} \theta^{2}-4 \eta \theta^{2}\right)\right)+\gamma^{2}\left(\eta^{2} \theta^{2}+8 b_{1}^{2}\left(-4 b_{2}+\theta^{2}\right)\right.\right. \\
& +4 b_{1}\left(6 \eta^{2}+b_{2} \theta^{2}-2 c_{2} \eta^{2} \theta^{2}+a_{1}\left(2 \eta\left(4 \eta-\theta^{2}\right)+8 b_{1}\left(-4 b_{2}+\theta^{2}\right)\right)+2 b_{1}\left(\left(-4 c_{0} \gamma-\left(c_{1}-4 c_{2}\right) \eta\right) \theta^{2}+a_{2}\left(\left(-8 \eta+4 \theta^{2}\right)\right)\right.\right. \\
& \left.\left.+2 b_{2}\left(8 c_{0} \gamma+c_{1} \theta^{2}-2 c_{2}\left(2 \eta+\theta^{2}\right)\right)\right)\right)+b_{0} \gamma\left(3 c_{0} \gamma \eta^{2} \theta^{2}+2 a_{1} b_{1}\left(16 b_{1} b_{2}-8 \eta^{2}-4 b_{1} \theta^{2}+3 \eta \theta^{2}\right)+2 b_{1}^{2}\left(\left(8 c_{0} \gamma+c_{1} \eta-4 c_{2} \eta\right) \theta^{2}\right.\right. \\
& \left.+a_{2}\left(8 \eta-4 \theta^{2}\right)+b_{2}\left(-32 c_{0} \gamma+8 c_{2} \eta-2 c_{1} \theta^{2}+4 c_{2} \theta^{2}\right)\right)+2 b_{1}\left(c_{0} \gamma\left(28 \eta^{2}+6 b_{2} \theta^{2}-15 \eta \theta^{2}\right)+\eta\left(\left(3 a_{2}+c_{1} \eta\right) \theta^{2}\right.\right. \\
& \left.\left.\left.\left.\left.\left.\left.+c_{2}\left(-8 \eta^{2}-3 b_{2} \theta^{2}+3 \eta \theta^{2}\right)\right)\right)\right)\right)\right)\right)\right) \cdot\left(4 \left(2 \gamma^{4}\left(8 b_{1} b_{2}-4 \eta^{2}-2 b_{1} \theta^{2}-b_{2} \theta^{2}+2 \eta \theta^{2}\right)-2 b_{0}^{2} b_{1}\left(\eta^{2} \theta^{2}+b_{1}^{2}\left(-8 b_{2}+2 \theta^{2}\right)\right.\right.\right. \\
& \left.\left.\left.+b_{1}\left(8 \eta^{2}+b_{2} \theta^{2}-4 \eta \theta^{2}\right)\right)+b_{0} \gamma^{2}\left(\eta^{2} \theta^{2}+8 b_{1}^{2}\left(-4 b_{2}+\theta^{2}\right)+4 b_{1}\left(6 \eta^{2}+b_{2} \theta^{2}-3 \eta \theta^{2}\right)\right)\right)\right)^{-1} .
\end{aligned}
$$

\section{Notations}

$w /$ unit: Unit wholesale price of the NB product $p_{0}$ /unit: Unit of direct selling price of the NB product $p_{1}$ /unit: Unit retail price of the NB product

$p_{2}$ /unit: Unit retail price of the SB product

$c_{0}$ : The marginal cost of production and operation in the direct channel

$c_{1}$ : The marginal production cost of the NB product

$c_{2}$ : The marginal production cost of the SB product

A: The advertising investment of the retailer

$u$ : $\quad$ Unit retail margin, $p_{1}=w+u$

$D_{0}$ : The demand function of the NB product in direct channel

$D_{1}$ : The demand function of the NB product in retail channel

$D_{2}$ : The demand function of the SB product in retail channel.

\section{Data Availability}

The data used to support the findings of this study are available from the corresponding author upon request.

\section{Conflicts of Interest}

The authors declare that they have no conflicts of interest.

\section{Acknowledgments}

The research was supported by the National Natural Science Foundation of China (no. 61273231 and no. 71571131).

\section{References}

[1] T. Xiao and J. Shi, "Pricing and supply priority in a dualchannel supply chain," European Journal of Operational Research, vol. 254, no. 3, pp. 813-823, 2016.

[2] H. Kurata, D.-Q. Yao, and J. J. Liu, "Pricing policies under direct vs. indirect channel competition and national vs. store brand competition," European Journal of Operational Research, vol. 180, no. 1, pp. 262-281, 2007.

[3] G. Cai, Z. G. Zhang, and M. Zhang, "Game theoretical perspectives on dual-channel supply chain competition with price discounts and pricing schemes," International Journal of Production Economics, vol. 117, no. 1, pp. 80-96, 2009.
[4] F. Soleimani, A. A. Khamseh, and B. Naderi, "Optimal decisions in a dual-channel supply chain under simultaneous demand and production cost disruptions," Annals of Operations Research, vol. 243, no. 1-2, pp. 301-321, 2016.

[5] R. Batarfi, M. Y. Jaber, and S. Zanoni, "Dual-channel supply chain: a strategy to maximize profit," Applied Mathematical Modelling, vol. 40, no. 21-22, pp. 9454-9473, 2016.

[6] Y. Liu and T. Xiao, "Pricing and collection rate decisions and reverse channel choice in a socially responsible supply chain with green consumers," IEEE Transactions on Engineering Management, pp. 1-13, 2019.

[7] B. Zheng, C. Yang, J. Yang, and M. Zhang, "Dual-channel closed loop supply chains: forward channel competition, power structures and coordination," International Journal of Production Research, vol. 55, no. 12, pp. 3510-3527, 2017.

[8] P. He, Y. He, and $\mathrm{H}$. Xu, "Channel structure and pricing in a dual-channel closed-loop supply chain with government subsidy," International Journal of Production Economics, vol. 213, pp. 108-123, 2019.

[9] B. C. Giri and S. K. Dey, "Game theoretic analysis of a closed-loop supply chain with backup supplier under dual channel recycling," Computers and Industrial Engineering, vol. 129, pp. 179-191, 2019.

[10] J. S. Raju, R. Sethuraman, and S. K. Dhar, "The introduction and performance of store brands," Management Science, vol. 41, no. 6, pp. 957-978, 1995.

[11] C. Narasimhan and R. T. Wilcox, "Private labels and the channel relationship: a cross-category Analysis," The Journal of Business, vol. 71, no. 4, pp. 573-600, 1998.

[12] D. E. Mills, "Why retailers sell private labels," Journal of Economics, vol. 4, no. 3, pp. 509-528, 1995.

[13] R. K. Tyagi, "Sequential product positioning under differential costs," Management Science, vol. 46, no. 7, pp. 928-940, 2000.

[14] F. Zhang and J. Ma, "Research on the complex features about a dual-channel supply chain with a fair caring retailer," Communications in Nonlinear Science and Numerical Simulation, vol. 30, no. 1-3, pp. 151-167, 2016.

[15] H. Liu, M. Lei, H. Deng, G. K. Leong, and T. Huang, “A dual channel, quality-based price competition model for the WEEE recycling market with government subsidy," Omega, vol. 59, pp. 290-302, 2016.

[16] Z. Liu, J. Tang, B. Li, and Z. Wang, "Trade-off between remanufacturing and recycling of WEEE and the environmental implication under the Chinese Fund Policy," Journal of Cleaner Production, vol. 167, pp. 97-109, 2017.

[17] W. Zhou, Y. Zheng, and W. Huang, "Competitive advantage of qualified WEEE recyclers through EPR legislation," European Journal of Operational Research, vol. 257, no. 2, pp. 641-655, 2017. 
[18] J. Ma and L. Xie, "The comparison and complex analysis on dual-channel supply chain under different channel power structures and uncertain demand," Nonlinear Dynamics, vol. 83, no. 3, pp. 1379-1393, 2016.

[19] Y. Dai, X. Chao, S. C. Fang, and H. L. W. Nuttle, "Pricing in revenue management for multiple firms competing for customers," International Journal of Production Economics, vol. 98, no. 1, pp. 1-16, 2005.

[20] D. Q. Yao, X. Yue, and J. J. Liu, "Vertical cost information sharing in a supply chain with value-adding retailers," Omega, vol. 36, no. 5, pp. 838-851, 2008.

[21] J. Ma, T. Li, and W. Ren, "Research on the complexity of dualchannel supply chain model in competitive retailing service market," International Journal of Bifurcation and Chaos, vol. 27, no. 7, Article ID 1750098, 2017.

[22] J. Ma and H. Ren, "Influence of government regulation on the stability of dual-channel recycling model based on customer expectation," Nonlinear Dynamics, vol. 94, no. 3, pp. 17751790, 2018. 